\title{
Quantum dilatonic gravity in $d=2,4$ and 5 dimensions
}

\author{
SHIN'ICHI NOJIRI and SERGEI D. ODINTSOV^? \\ Department of Mathematics and Physics \\ National Defence Academy, Hashirimizu Yokosuka 239, JAPAN \\ ه Instituto de Fisica de la Universidad de Guanajuato, \\ Lomas del Bosque 103, Apdo. Postal E-143, 37150 Leon, Gto., MEXICO \\ and \\ Tomsk State Pedagogical University, 634041 Tomsk, RUSSIA
}

\begin{abstract}
We review (mainly) quantum effects in the theories where gravity sector is described by metric and dilaton. The one-loop effective action for dilatonic gravity in two and four dimensions is evaluated. Renormalization group equations are constructed. The conformal anomaly and induced effective action for $2 \mathrm{~d}$ and $4 \mathrm{~d}$ dilaton coupled theories are found. It is applied to study of quantum aspects of black hole thermodynamics, like calculation of Hawking radiation and quantum corrections to black hole parameters and investigation of quantum instability for such objects with multiple horizons. The use of above effective action in the construction of non-singular cosmological models in Einstein or Brans-Dicke (super)gravity and investigation of induced wormholes in supersymmetric Yang-Mills theory are given.

5 d dilatonic gravity (bosonic sector of compactified IIB supergravity) is discussed in connection with bulk/boundary (or AdS/CFT) correspondence. Running gauge coupling and quark-antiquark potential for boundary gauge theory at zero or non-zero temperature are calculated from $d=5$ dilatonic Anti-de Sitter-like background solution which represents Anti-de Sitter black hole for periodic time.
\end{abstract}

\footnotetext{
${ }^{1}$ e-mail: nojiri@cc.nda.ac.jp, snojiri@yukawa.kyoto-u.ac.jp

2e-mail: odintsov@ifug5.ugto.mx, odintsov@mail.tomsknet.ru
} 


\section{Contents}

\begin{tabular}{lll}
\hline 1 & Introduction: Why dilatonic gravity? & 1
\end{tabular}

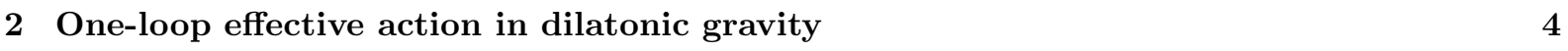

$2.1 \quad$ Perturbative renormalization of two-dimensional dilatonic gravity $\ldots \ldots \ldots \ldots$

2.2 Quantum equivalence in the models of 2 d dilatonic gravity $\ldots \ldots \ldots$. . . . . . . . . 10

2.3 Dilatonic gravity with matter in $2+\epsilon$-dimensions . . . . . . . . . . . . . . . . . 13

$2.4 \quad$ One-loop effective action in $4 \mathrm{~d}$ higher derivative dilatonic gravity $\ldots \ldots \ldots \ldots$

3 Conformal anomaly and induced effective action for dilaton coupled theories $\quad 20$

3.1 2d dilaton coupled scalar and quantum dilaton: conformal anomaly and induced action . 20

3.2 Dilaton coupled spinor conformal anomaly in two dimensions . . . . . . . . . . . . . 23

3.3 Induced effective action for 4 d dilaton coupled scalar $\ldots \ldots \ldots \ldots$. . . . . . . . . 25

3.4 Conformal and chiral anomaly for $4 \mathrm{~d}$ dilaton coupled spinor $\ldots \ldots \ldots \ldots$

3.5 Conformal anomaly for $4 \mathrm{~d}$ dilaton coupled vector field $\ldots \ldots \ldots$. . . . . . . . . . . 27

3.6 Conformal anomaly and induced action in 2d dilatonic supergravity with dilaton coupled

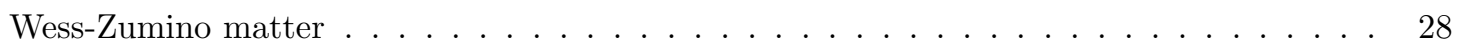

\begin{tabular}{|lll}
\hline & Thermodynamics of quantum dilatonic black holes & $\mathbf{3 0}$
\end{tabular}

$4.12 \mathrm{~d}$ dilatonic black holes and Hawking radiation $\ldots \ldots \ldots \ldots \ldots \ldots \ldots$

4.2 Thermodynamics of Schwarzschild-de Sitter black holes $\ldots \ldots \ldots$. . . . . . . . . . 34

4.3 Anti-evaporation of multiply horizon black holes $\ldots \ldots \ldots \ldots$. . . . . . . . . 40

\begin{tabular}{|ll|l}
5 Quantum cosmology & 45
\end{tabular}

5.1 (Non)-singular Kantowski-Sachs Universe $\ldots \ldots \ldots \ldots$. . . . . . . . . . . 46

5.2 Inflationary Brans-Dicke quantum Universe $\ldots \ldots \ldots \ldots \ldots \ldots$

5.3 Primordial wormholes at the early Universe $\ldots \ldots \ldots \ldots \ldots \ldots$

6 Dilatonic gravity and Anti-de Sitter/Conformal Field Theory correspondence $\quad 56$

6.1 Axion-dilatonic conformal anomaly from AdS/CFT correspondence . . . . . . . . . . . 56

6.2 Running gauge coupling and quark-antiquark potential from IIB supergravity . . . . . . . 61

6.3 Dilatonic AdS black holes: running gauge coupling and $q \bar{q}$-potential at non-zero temperature 66

6.4 Strong coupling limit of $\mathcal{N}=2$ superconformal field theory free energy from AdS black hole thermodynamics . . . . . . . . . . . . . . . . . . . . 71

\begin{tabular}{lll}
\hline 7 & Discussion & 73
\end{tabular} 


\section{Introduction: Why dilatonic gravity?}

Dilatonic gravity represents very natural generalization of general relativity where gravitational sector is described by metric tensor and also by the scalar partner (dilaton). In principle, any covariant theory of gravity interacting in some way with scalar which is assumed to describe not matter may be called the dilatonic gravity. The simplest form of dilatonic gravity is given by general relativity plus scalar field Lagrangian where scalar sector may contain kinetic term and some potential. It is then clear that for constant scalar such a theory is reduced to general relativity (with cosmological constant in case of presence of scalar potential). One may think about more complicated generalizations where curvature non-minimally couples with scalar.

Historically, one of the first theories of this sort has been suggested by Brans and Dicke. It is still considered as an alternative to general relativity. Its action in so-called Jordan frame is given as

$$
S_{B D}=\frac{1}{16 \pi} \int d^{4} x \sqrt{-g}\left[\phi R-\frac{\omega}{\phi}\left(\nabla_{\mu} \phi\right)\left(\nabla^{\mu} \phi\right)\right]+S_{M},
$$

where $\phi$ is scalar (Brans-Dicke dilaton), $R$ is curvature, $\omega$ is Brans-Dicke parameter and $S_{M}$ is usual matter action. As one can see in Jordan frame the gravitational field is described by the metric tensor and by dilaton.

The important role in dilatonic gravity is played by conformal transformations. Generally speaking, starting from the spacetime $M$ with metric $g_{\mu \nu}$ one can make (non-singular) coordinates dependent rescaling of metric $g_{\mu \nu} \rightarrow \tilde{g}_{\mu \nu}=\exp (\sigma(x)) g_{\mu \nu}$ where $\sigma(x)$ is some arbitrary function of coordinates. Such rescaling is called Weyl or conformal transformation. It leaves the light cones unchanged, in other words, the spacetime $M$ with metric $g_{\mu \nu}$ and the one with metric $\tilde{g}_{\mu \nu}$ have the same causal structure.

This conformal transformation is extremely important as it permits to map one classical theory to another theory which is sometimes easier to study. The explicit example of such mapping (dilaton is also transformed) is given in section 5.2 (see also section 2.1) where Brans-Dicke-matter theory in Jordan frame is transformed to the one in Einstein frame. As a result, in gravity sector the curvature appears as in Einstein theory (minimal coupling with dilaton) while matter is getting dilaton coupled. There may be given arguments that Einstein frame is the physical one.

Conformal transformations are especially important in conformal quantum theory. It means that on classical level such theories do not change under conformal transformations. The manifestation of this symmetry on the conservation laws level is the vanishing of energy-momentum tensor trace, i.e. $T_{\mu}{ }^{\mu}=0$. However, on quantum level one needs to apply some regularization and (or) renormalization for calculation of formally divergent, infinite quantities. Preserving covariance in such regularized calculations, one breaks the conformal invariance on quantum level. As a result, trace of quantum energy-momentum tensor is not zero. There appears so-called conformal or Weyl anomaly (for a review, see [1]). The practical importance of conformal anomaly is related with the possibility to integrate over it and to construct the anomaly induced, finite effective action. (In other words, instead of taking into account quantum effects one is forced to add to classical gravitational action some (non-local) finite gravitational action which is classical one and which role is to describe the effects related with quantum theory). This property will be extensively used in the present review for conformally invariant, dilaton coupled theories 
where anomaly induced effective action on the arbitrary dilaton-gravitational background is found. (Note that usually one can calculate the effective action or on some specific background or approximately, see book [2] for a review).

Another big class of theories where classical dilatonic gravity naturally appears is related with the process of compactification (or reduction). As an example, let us consider six-dimensional Einstein gravity (with cosmological constant). Imagine that one is looking to the background of the sort: $M_{4} \times S_{2}$ where $M_{4}$ is an arbitrary four-dimensional manifold and $S_{2}$ is two-dimensional sphere with the radius $r$ which depends explicitly from the coordinates of the space $M_{4}$. The metric of the background $M_{4} \times S_{2}$ may be substituted to the action of above six-dimensional Kaluza-Klein gravity. After integration over coordinates of two-dimensional sphere one is left with effective $4 \mathrm{~d}$ dilatonic gravity of special form where Kaluza-Klein dilaton (scalar) originates from the radius of $S_{2}$ and $6 \mathrm{~d}$ cosmological constant (if presents) gives rise to dilatonic potential. Similarly, after spherical (or hyperbolic, or torus) reduction of $4 \mathrm{~d}$ Einstein gravity one gets two-dimensional dilatonic gravity. That is why in many cases usual four-dimensional backgrounds of Einstein gravity may be also understood as $2 \mathrm{~d}$ dilatonic backgrounds. In this sense, standard Schwarzschild black hole (no dilaton) is the same as 2d dilatonic black hole of special form. Then, the calculations performed for $2 \mathrm{~d}$ dilatonic gravity may very often give the answers for $4 \mathrm{~d}$ Einstein gravity and vice-versa.

From another side this property gives additional motivation to study the dilatonic gravity. Indeed, even the hypothetical proof of non-existence of dilaton in Nature would be given one may still work with $2 \mathrm{~d}$ dilatonic gravity having in mind that this is just another way to describe Einstein gravity after reduction. Similarly, if Universe was multi-dimensional at some stage one can argue the presence of Kaluza-Klein dilaton at early Universe. Of course, such dilaton should quickly decay.

Nevertheless, the most natural way to introduce the dilaton comes presumably from supergravity and (super)strings. First of all, in many cases supersymmetry dictates the presence of dilaton in supermultiplet as in $2 \mathrm{~d}$ dilatonic supergravity of section 3.6 or IIB supergravity in section 6.2. For strings the situation is even more dramatical: all versions of string theory predict the presence of scalar partner to usual tensor graviton. It is assumed that such scalar should acquire a mass (thanks to some, not yet well-understood dynamical mechanism). Then, macroscopic consequences of Einstein and string dilaton gravity are more-less equivalent. Otherwise, if dilaton is massless the theory may become non-consistent due to possible violation of (weak) equivalence principle and cosmological variations of gauge coupling constants, etc.(However, some mechanisms to make theory consistent even for massless dilaton may be suggested). We will not discuss this circle of questions here as we are mainly dealing with quantum dilatonic gravity but not with macroscopic manifestations. Moreover, even for classical dilatonic gravity there remain still some unresolved questions. For example, the dilaton may serve sometimes as panacea to explain new observational effects like Pioner effect[3].

The purpose of this work is to give the review of results and methods in (mainly quantum) dilatonic gravity in various dimensions. We attempt to present the details of typical calculations in the given examples in a self-consistent way so that in most cases there is no necessity to consult original works in order to understand the main points. 
In the next chapter we review the background field method evaluation of the divergent part of the oneloop effective action in $2 \mathrm{~d}$ and $4 \mathrm{~d}$ dilatonic gravities where dilaton is dimensionless scalar. Consideration is done of general (non-renormalizable) model of $2 \mathrm{~d}$ dilatonic gravity (with matter) which includes stringinspired models and the ones reduced from Einstein gravity. The obtained one-loop effective action is applied to show that $2 \mathrm{~d}$ dilatonic gravities which are classically equivalent are not equivalent on quantum level, generally speaking. The equivalence is restored only on-shell. That alerts on the danger of making quantum calculations in convenient parameterization and then using results of these calculations in another (physical) parameterization of fields. As another application of the effective action we give the renormalization group study of dilatonic gravity near two dimensions, using $\epsilon$-expansion technique. It is shown that theory under consideration may have stable fixed points for all coupling functions.

In the third chapter the calculation of conformal anomaly for Weyl invariant, dilaton coupled $2 \mathrm{~d}$ and $4 \mathrm{~d}$ scalars and spinors as well as for $4 \mathrm{~d}$ dilaton coupled vector is presented. Such $n$-dimensional dilaton coupled matter appears from usual $n+2$-dimensional matter (no dilaton) after reduction. Integrating over such conformal anomaly one is able to construct finite, anomaly induced action which is not limited to some specific background. This is very powerful method to get the effective action on the arbitrary dilaton-gravitational background. Unfortunately, it cannot be applied to dilatonic gravity itself as such theory is not, as a rule, Weyl invariant. Of course, working in large $N$ approximation one can neglect the proper quantum gravity contribution to the effective action. As one example the large $N$, anomaly induced effective action is presented for $2 \mathrm{~d}$ dilatonic supergravity with dilaton coupled Wess-Zumino model.

Fourth chapter is devoted to study of quantum aspects of (dilatonic) black holes using above induced effective action. The overview of two simple models of $2 \mathrm{~d}$ dilatonic black holes where quantum effects of minimal matter induce Hawking radiation is given. In these models the dilaton is classical one as it does not appear in quantum effective action. Then, the calculation of quantum corrections to thermodynamics of 4d Schwarzschild and Schwarzschild-(anti) de Sitter black hole is presented where effective action of third chapter (in large $N$ and $s$-wave approximation) is extensively used. In particular, quantum corrections to black hole horizon radii, temperature, mass and entropy are found. The important property of such results is that such quantum corrections are also the quantum corrections to $2 \mathrm{~d}$ dilatonic black holes. This double role of results is discussed. At the final section of fourth chapter we discuss the effect of anti-evaporation (i.e. increase of horizon radius) of multiply horizon black holes due to quantum effects of matter. As an example, again Schwarzschild-de Sitter and $2 \mathrm{~d}$ charged dilatonic black holes are taken and anomaly induced effective action is used. Above effect is opposite to the well-known Hawking radiation.

Fifth chapter is devoted to presentation of some elements of (dilatonic) quantum cosmology. Again with large $N$ anomaly induced effective action due to quantum conformally invariant matter we construct non-singular 4d Kantowski-Sachs Universe. Such Universe may be also presented as 2d dilatonic cosmology. For 2d dilatonic supergravity (or some its modifications) with Wess-Zumino matter the role of quantum effects of matter to early Universe evolution is carefully discussed. For Brans-Dicke theory with dilaton coupled matter (Einstein frame) the quantum matter effects lead to the possibility of infla- 
tionary Universe with quickly decaying dilaton. In the last section we show the possibility to induce the primordial wormholes which are hard to realize in terms of classical gravity by quantum effects of Grand Unified Theories in the early Universe.

In the last, sixth chapter we study mainly classical aspects of odd-dimensional (mainly five-dimensional) dilatonic gravity descending from IIB supergravity (bosonic sector). While we look for classical Anti-de Sitter-like solutions of such theory and it may look distant from quantum dilatonic gravity, it is not so. The reason is well-accepted now bulk/boundary correspondence. With it from classical solutions of IIB supergravity we get the description of dual gauge theory which lives on the boundary. In the first section of this chapter from one approximate solution of $3 \mathrm{~d}$ and $5 \mathrm{~d}$ axion-dilatonic gravity we get correct conformal anomaly studied from quantum field theory point of view in chapter 3 for $2 \mathrm{~d}$ dilaton coupled theories and for maximally supersymmetric Yang-Mills theory conformally coupled with conformal supergravity. In the next section we present the $5 \mathrm{~d}$ dilatonic solution interpolating between Anti-de Sitter space and flat space with singular dilaton. This solution may be interpreted as the one describing running gauge coupling in boundary gauge theory. In addition, the Wilson loop is obtained and the possibility of confinement is mentioned. The generalization of this solution (approximate Anti-de Sitter black hole) to include non-zero temperature is done. Then, from calculation of entropy and free energy of such 5d black hole one can get the entropy and free energy of boundary gauge theory at strong coupling limit. The role of higher derivative terms in such correspondence is discussed and free energy of super Yang-Mills theory (with two supersymmetries) is found.

Some resume and open questions are listed in the Discussion. In this review, we mainly use the following conventions of curvatures :

$$
\begin{aligned}
& R=g^{\mu \nu} R_{\mu \nu}, \quad R_{\mu \nu}=-\Gamma_{\mu \lambda, \kappa}^{\lambda}+\Gamma_{\mu \kappa, \lambda}^{\lambda}-\Gamma_{\mu \lambda}^{\eta} \Gamma_{\kappa \eta}^{\lambda}+\Gamma_{\mu \kappa}^{\eta} \Gamma_{\lambda \eta}^{\lambda} \\
& \Gamma_{\mu \lambda}^{\eta}=\frac{1}{2} g^{\eta \nu}\left(g_{\mu \nu, \lambda}+g_{\lambda \nu, \mu}-g_{\mu \lambda, \nu}\right)
\end{aligned}
$$

and we choose the Einstein action in the following form :

$$
S_{\text {Einstein }}=-\frac{1}{\kappa^{2}} \int d^{d} x \sqrt{-g} R
$$

However, in some cases we use also Euclidean notations

$$
S_{\text {Euclid }}=\frac{1}{\kappa^{2}} \int d^{d} x \sqrt{g} R
$$

The reason is that we follow to results of different works, often done with different notations.

\section{One-loop effective action in dilatonic gravity}

In the present Chapter we discuss the perturbative approach to dilatonic gravity. The one-loop effective action is calculated and renormalization structure is discussed. Some immediate consequences of the effective action like renormalization group equations near two and in four dimensions are presented. The comparison of off-shell and on-shell ( S-matrix) effective actions for classically equivalent models is made. 


\subsection{Perturbative renormalization of two-dimensional dilatonic gravity}

In this section, we discuss the covariant effective action corresponding to a very general, multiplicatively renormalizable 田, 5, 6, (in generalized sense) model of $2 \mathrm{~d}$ dilatonic gravity with matter. Its action has the following form

$$
S=-\int d^{2} x \sqrt{g}\left[\frac{1}{2} Z(\Phi) g^{\mu \nu} \partial_{\mu} \Phi \partial_{\nu} \Phi+C(\Phi) R-\frac{1}{2} f(\Phi) g^{\mu \nu} \partial_{\mu} \chi_{i} \partial_{\nu} \chi_{i}+V(\Phi, \chi)\right] .
$$

It includes a dilaton field $\Phi$ and real scalar $\chi_{i}$.

This action describes and generalizes many well-known dilaton models. For instance, the celebrated bosonic string effective action corresponds to

$$
Z(\Phi)=8 \mathrm{e}^{-2 \Phi}, C(\Phi)=\mathrm{e}^{-2 \Phi}, V(\Phi)=4 \lambda^{2} \mathrm{e}^{-2 \Phi}, f(\Phi)=1 .
$$

On the other hand, in the absence of matter our action for

$$
Z=1, \quad C(\Phi)=\Phi, \quad V(\Phi)=\Lambda \Phi,
$$

coincides with the Jackiw-Teitelboim action [8, 9].

In principle, at the classical level the theory defined by the action (5) can be transformed into an equivalent theory whose corresponding action is more simple. Indeed, this can be done by choosing the field $\varphi_{1}$ as defined through the equation

$$
Z^{1 / 2}(\Phi) \partial_{\mu} \Phi=\partial_{\mu} \varphi_{1}
$$

and by expressing $\Phi$ as $\Phi=\Phi\left(\varphi_{1}\right)$. Next, let us introduce a new field, $\varphi_{2}$, via

$$
c \varphi_{2}=C\left(\Phi\left(\varphi_{1}\right)\right)
$$

and write then $\Phi=\Phi\left(\varphi_{2}\right)$. After having done this, by making the transformation 10$]$

$$
g_{\mu \nu} \longrightarrow \mathrm{e}^{2 \rho\left(\varphi_{2}\right)} \bar{g}_{\mu \nu}
$$

with a properly chosen $\rho\left(\varphi_{2}\right)$ [10], one can see that the theory (5) with the transformed metric (10) is classically equivalent to more simple, particular case

$$
Z=1, \quad C=c \varphi_{2}, \quad V=\mathrm{e}^{2 \rho\left(\varphi_{2}\right)} V\left(\varphi_{2}, \chi\right), \quad f(\Phi)=f\left(\varphi_{2}\right) .
$$

However, the model (11) (which, generally speaking, may be considered as a representative of the general class (5)) is still complicated enough. Moreover, it still includes arbitrary functions of the dilaton (now $\varphi_{2}$ ), as (5) does. Finally, the classical equivalence may be lost at the quantum level. For all these reasons, we choose to consider the quantum effective action corresponding to more general theory (5).

Let us briefly remind the definition and the properties of the effective action. Let $\phi$ is set of fields, $S(\phi)$ is classical action. Imagine for the moment that classical fields $\phi$ are not gauge fields. Then, the effective action $\Gamma(\phi)$ is defined via the following integro-differential equation:

$$
\exp \left(\frac{i}{\hbar} \Gamma(\phi)\right)=\int \mathrm{D} \varphi\left[\frac{i}{\hbar}\left(S[\varphi+\phi]-\int d^{4} x \varphi(x) \frac{\delta \Gamma(\phi)}{\delta \phi(x)}\right)\right] .
$$


Here $\hbar$ is Planck constant, $\varphi$ is set of quantum fields corresponding to $\phi$. Actually, to derive the above equation, one uses the background field method where the quantum fields transformation $\varphi \rightarrow \varphi+\phi$ is supposed. Here, $\varphi$ are called quantum fields and $\phi$ are called the background fields. In case when gauge fields are present, the correspoonding gauge-fixing term should be included into the classical action. It also induces the so-called ghosts action. We will not give more details of presentation of effective action for gauge fields as it may be found in the most of modern textbooks on quantum field theory. It is enough to consider the effective action for non-gauge theories to demonstrate the qualitative properties.

The equation (12) may be further represented as follows (after the functional Taylor series expansion of $S[\phi+\varphi]$ is done):

$$
\begin{aligned}
\exp \left(\frac{i}{\hbar} \Gamma(\phi)\right)= & \int \mathrm{D} \varphi \exp \left\{\frac { i } { \hbar } \left(S(\phi)+\frac{1}{2} S_{2}(\phi) \varphi^{2}\right.\right. \\
& \left.\left.+\sum_{n=3}^{\infty} \frac{\hbar^{\frac{n}{2}-1}}{n !} S_{n}(\phi) \varphi^{n}-\hbar^{-\frac{1}{2}} \varphi\left(\Gamma_{1}(\phi)-S_{1}(\phi)\right)\right)\right\}
\end{aligned}
$$

Here $S_{n}=\frac{\delta^{n} S(\phi)}{\delta \phi\left(x_{1}\right) \cdots \delta \phi\left(x_{n}\right)}, \varphi \Gamma(\phi)=\int d^{4} x \varphi(x) \frac{\delta \Gamma(\phi)}{\delta \phi(x)}$. Eq. 13) gives the loop expansion of the effective action. Limiting to only one-loop approximation (second term in right hand side of Eq.(13)), introducing the notation

$$
\Gamma(\phi)=S(\phi)+\bar{\Gamma}(\phi)=S(\phi)+\sum_{n=1}^{\infty} \hbar^{n} \bar{\Gamma}^{(n)}(\phi)
$$

and making functional integration over quadratic on $\varphi$ terms one gets;

$$
\Gamma^{(1)}(\phi)=S(\phi)+\bar{\Gamma}^{(1)}(\phi)=S(\phi)+\frac{i}{2} \operatorname{Tr} \log S_{2}(\phi) .
$$

Eq.(15) gives the one-loop effective action for non-gauge theories. For gauge theories, the key idea is very similar, but due to difference in technique more Tr log terms (ghosts contribution) appear. As we will be dealing with only one-loop approximation, we often do not write explicitly the index "1" or hat under $\Gamma(\phi)$.

It is usually happens in QFT that loop corrections to the effective action are divergent (they contain infinities). To avoid these divergences the standard prescription is to use some regularization. For example, in one-loop approximation we can present

$$
\bar{\Gamma}^{(1)}(\phi)=\Gamma_{f i n}^{(1)}(\phi)+\Gamma_{d i v}^{(1)}(\phi) .
$$

Depending on regularization under consideration, now the separation to finite and divergent pieces in Eq.(16) is ambiguous. The simplest regularization could be just omitting of the divergent part in Eq.(16).

There is one extremely important case of the theories where the structure of divergences of effective action (in all orders of loop expansion) repeats the structure of classical action. In this case (for details, one can consult any modern textbook on QFT) $\Gamma_{\text {div }}(\phi)$ which may be calculated via Schwinger-De Witt expansion is in some sense universal and represents the main interest. First of all, the explicit calculation of $\Gamma_{d i v}$ (which can be done normally only at one-loop, at best at two- or three-loops) indicates explicitly if theory is renormalizable or not. This is especially important for non-linear theories like (dilatonic) gravity. Second, if theory is renormalizable (even at one-loop) then $\Gamma_{d i v}$ defines beta-functions. Using 
these beta-functions (for details, see [2]) one can construct the finite part of effective action (actually, even in better approximation as so-called RG improvement may be applied). The example of such finite effective action which satisfies to RG equations is presented in Section 2.4. Third, divergent part of effective action maybe used in the construction of conformal anomaly as we see in next Chapter.

Taking into account above remarks, we give the explicit calculation of one-loop effective action divergences in dilatonic gravity (with matter). We show that theory could be one-loop renormalizable but only for special values of dilatonic couplings and dilatonic potential. In this case, finite part of effective action may be restored also. Before going to direct calculation, let us briefly describe the generalization of above effective action for gauge theories (in background gauge) as this is the case of dilatonic gravity.

Having the gauge theory with action $S(\phi)$, closed algebra and linearly independent generators one introduces the generating functional of connected Green functions as follows:

$$
\begin{aligned}
\exp [i W[J, \tilde{\phi}]]= & \int \mathrm{D} \varphi \mathrm{D} \bar{C} \mathrm{D} C \exp \{i(S(\varphi) \\
& +\frac{1}{2} G_{\alpha \beta}[\tilde{\phi}] t_{i}^{\alpha}[\tilde{\phi}] t_{j}^{\beta}[\tilde{\phi}] \varphi^{i} \varphi^{j} \\
& \left.\left.+\bar{C}_{\alpha} t_{i}^{\alpha}[\tilde{\phi}] R_{\beta}^{i}[\tilde{\phi}] C^{\beta}-\frac{1}{2} \operatorname{Tr} \log G_{\alpha \beta}[\tilde{\phi}]+\varphi^{i} J_{i}\right)\right\}
\end{aligned}
$$

where $\varphi^{i}$ is set of gauge fields, $\tilde{\phi}^{i}$ are correspondent background fields, $R_{\alpha}^{i}$ are generators of gauge group, linear gauge is chosen: $\chi^{\alpha}[\varphi]=t_{i}^{\alpha} \varphi^{i}, C^{\alpha}, \bar{C}^{\alpha}$ are ghost and antighost. The Planck constant is chosen to be equal to unity. The gauge dependence is defined through the choice of functions $G_{\alpha \beta}, t_{i}^{\alpha}$. One also defines the mean field $\bar{\phi}^{i}$ :

$$
\bar{\phi}^{i}=\frac{\delta W[J, \tilde{\phi}]}{\delta J^{i}} .
$$

The effective action is introduced via Legendre transformation:

$$
\Gamma[\bar{\phi}, \tilde{\phi}]=W[J, \tilde{\phi}]-\bar{\phi}^{i} J_{i}
$$

where the source $J_{i}$ is expressed in terms of mean field.

Changing the variable $\varphi \rightarrow \varphi+\bar{\phi}$ in Eq.(17) and putting $\bar{\phi}=\tilde{\phi}$ as special condition, one can get

$$
\begin{aligned}
\exp (i \Gamma[\bar{\phi}])= & \int \mathrm{D} \varphi \mathrm{D} \bar{C} \mathrm{D} C \exp \{i(S(\bar{\phi}+\varphi) \\
& +\frac{1}{2} G_{\alpha \beta}[\bar{\phi}] t_{i}^{\alpha}[\bar{\phi}] t_{j}^{\beta}[\bar{\phi}]\left(\bar{\phi}^{i}+\varphi^{i}\right)\left(\bar{\phi}^{j}+\varphi^{j}\right) \\
& \left.\left.+\bar{C}_{\alpha} t_{i}^{\alpha}[\bar{\phi}] R_{\beta}^{i}[\bar{\phi}+\varphi] C^{\beta}-\frac{1}{2} \operatorname{Tr} \log G_{\alpha \beta}[\bar{\phi}]+\left.\varphi^{i} \frac{\delta \Gamma[\bar{\phi}, \tilde{\phi}]}{\delta \varphi^{i}}\right|_{\bar{\phi}=\tilde{\phi}}\right)\right\}
\end{aligned}
$$

where $\Gamma[\bar{\phi}]=\left.\Gamma[\bar{\phi}, \tilde{\phi}]\right|_{\tilde{\phi}=\bar{\phi}}$. This effective action in background gauge represents the generalization of the one for non-gauge theories in Eq.(12). It is important that one can prove that

$$
\bar{\Gamma}_{, i}[\bar{\phi}] R_{\alpha}^{i}[\bar{\phi}]=0
$$

In other words, such effective action is gauge invariant one. The above approach is called background field method. 
In the one-loop approximation it may be found in analogy with Eq.(15) as follows:

$$
\begin{aligned}
\bar{\Gamma}^{(1)}[\bar{\phi}]= & \frac{i}{2} \operatorname{Tr} \log \left\{S_{, i j}[\bar{\phi}]+G_{\alpha \beta}[\bar{\phi}] t_{i}^{\alpha}[\bar{\phi}] t_{j}^{\beta}[\bar{\phi}]\right\} \\
& -i \operatorname{Tr} \log \left\{t_{i}^{\alpha}[\bar{\phi}] R_{\beta}^{i}[\bar{\phi}]\right\}-\frac{i}{2} \operatorname{Tr} \ln G_{\alpha \beta}[\bar{\phi}] .
\end{aligned}
$$

Second term in Eq.22 gives the one-loop ghosts contribution while third term usually gives no contribution to divergent part of the effective action (except the case of higher derivative theories). As one sees below the Eq.(22) (no last term) is typical one-loop divergent EA structure in dilatonic gravity.

Let us now briefly describe the calculation of one-loop effective action in dilatonic gravity (5). The covariant gauge fixing condition is chosen as follows (see also next section,Eq.(42) and below for definition of background and quantum fields)

$$
S_{g . f .}=-\frac{1}{2} \int c_{\mu \nu} \chi^{\mu} \chi^{\nu}, \quad \chi^{\mu}=-\nabla_{\nu} \bar{h}^{\mu \nu}+\frac{C^{\prime}}{C} \nabla^{\mu} \varphi, \quad c_{\mu \nu}=-C \sqrt{g} g_{\mu \nu},
$$

The total quadratic contribution to the action takes the form

$$
S_{t o t}^{(2)}=-\frac{1}{2} \int d^{2} x \sqrt{g} \phi^{i} \widehat{H}_{i j} \phi^{j},
$$

where $\widehat{H}$ is the second order minimal operator, and $\phi^{i}$ is the set of all quantum fields including graviton, dilaton and matter fields (if present). The one-loop effective action in terms of supertraces becomes

$$
\Gamma=\frac{i}{2} \operatorname{Tr} \log \widehat{H}-i \operatorname{Tr} \log \widehat{\mathcal{M}},
$$

where $\widehat{H}=-\widehat{K} \Delta+\widehat{L}^{\lambda} \nabla_{\lambda}+\widehat{P}$, and the last term in (24) is the ghost operator corresponding via (23) to diffeomorphisms. Here $\widehat{K}$ and $\widehat{L}$ and $\widehat{P}$ are some matrices which form follows from making second

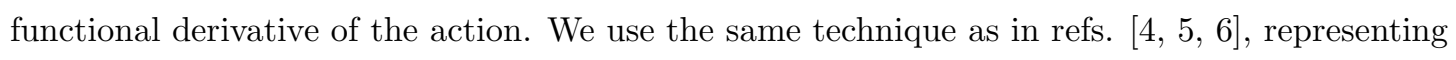

$$
\widehat{H}=-\widehat{K}\left(\widehat{1} \Delta+2 \widehat{E}^{\lambda} \nabla_{\lambda}+\widehat{\Pi}\right), \quad \widehat{E}^{\lambda}=-\frac{1}{2} \widehat{K}^{-1} \widehat{L}^{\lambda}, \quad \widehat{\Pi}=-\widehat{K}^{-1} \widehat{P} .
$$

After that, the standard algorithm can be used, namely

$$
\frac{i}{2} \operatorname{Tr} \log \widehat{H}=\left.\frac{i}{2} \operatorname{Tr} \log \left(\widehat{1} \Delta+2 \widehat{E}^{\lambda} \nabla_{\lambda}+\widehat{\Pi}\right)\right|_{d i v}=\frac{1}{2 \epsilon} \int d^{2} x \sqrt{g} \operatorname{Tr}\left(\widehat{\Pi}+\frac{R}{6} \widehat{1}_{-} \widehat{E}^{\lambda} \widehat{E}_{\lambda}-\nabla_{\lambda} \widehat{E}^{\lambda}\right),
$$

where $\epsilon=2 \pi(n-2)$. To obtain the divergent part, $\Gamma_{2-d i v}$, we have to evaluate the functional traces of the matrices above according to (26). After some tedious algebra [6] we arrive at

$$
\begin{aligned}
\Gamma_{2-d i v}=\frac{i}{2} \operatorname{Tr} \log \widehat{H}=-\frac{1}{2 \epsilon} \int d^{2} x \sqrt{g}\left\{\frac{4}{3} R\right. & +\frac{2}{C} V+\frac{2}{C^{\prime}} V^{\prime}+\left(\frac{2 C^{\prime}}{C}-\frac{Z}{C^{\prime}}\right)(\Delta \Phi) \\
& \left.-\left(\frac{3 C^{\prime 2}}{2 C^{2}}+\frac{C^{\prime \prime} Z}{C^{\prime 2}}\right)\left(\nabla^{\lambda} \Phi\right)\left(\nabla_{\lambda} \Phi\right)\right\}
\end{aligned}
$$

To complete the calculation one has to consider the ghost operator

$$
\widehat{\mathcal{M}}_{\nu}^{\mu} \equiv \frac{\delta_{R} \chi^{\mu}}{\delta \phi^{j}} \nabla_{\nu}^{j}
$$

where $\delta_{R}$ stands for the right functional derivative and the generators of the diffeomorphisms are

$$
\nabla_{\nu}^{\varphi}=-\left(\nabla_{\nu} \Phi\right), \quad \nabla_{\nu}^{\bar{h}_{\rho \sigma}}=g_{\rho \sigma} \nabla_{\nu}-g_{\nu \rho} \nabla_{\sigma}-g_{\nu \sigma} \nabla_{\rho}
$$


Explicitly,

$$
\widehat{\mathcal{M}}_{\nu}^{\mu}=g_{\nu}^{\mu} \Delta-\frac{C^{\prime}}{C}\left(\nabla_{\nu} \Phi\right) \nabla^{\mu}-\frac{C^{\prime}}{C}\left(\nabla^{\mu} \nabla_{\nu} \Phi\right)+R_{\nu}^{\mu}
$$

which leads to

$$
\Gamma_{g h-d i v}=-\frac{1}{2 \epsilon} \int d^{2} x \sqrt{g}\left\{\frac{8}{3} R-\frac{C^{\prime}}{C}(\Delta \Phi)+\left(\frac{C^{\prime \prime}}{C}-\frac{3 C^{\prime 2}}{2 C^{2}}\right)\left(\nabla^{\lambda} \Phi\right)\left(\nabla_{\lambda} \Phi\right)\right\} .
$$

The final answer is $[4,6]$

$$
\begin{aligned}
\Gamma_{d i v}=-\frac{1}{2 \epsilon} \int d^{2} x \sqrt{g}\{4 R+ & \frac{2}{C} V+\frac{2}{C^{\prime}} V^{\prime}+\left(\frac{C^{\prime}}{C}-\frac{Z}{C^{\prime}}\right)(\Delta \Phi) \\
& \left.+\left(\frac{C^{\prime \prime}}{C}-3 \frac{C^{\prime 2}}{C^{2}}-\frac{C^{\prime \prime} Z}{C^{\prime 2}}\right)\left(\nabla^{\lambda} \Phi\right)\left(\nabla_{\lambda} \Phi\right)\right\} .
\end{aligned}
$$

Notice that all surface terms have been kept in Eq. (31). Similar calculation of one-loop effective action in $2 \mathrm{~d}$ dilatonic gravity (without matter) has been discussed also in refs. [11, 12, 13, 10, 14].

Having performed the above calculation, now it is not difficult to take into account the scalar fields $\chi_{i}$, and to repeat it for the effective action (5). In fact, the introduction of the scalars leads to minor changes. In the background field notation $\chi_{i} \rightarrow \chi_{i}+\sigma_{i}$ the quantum fields are $\phi^{i}=\left\{\varphi ; h ; \bar{h}_{\mu \nu} ; \sigma_{i}\right\}$. Scalars do not spoil the minimality of the second functional derivative operator, so that the gauge condition and ghost action may be left untouched. The 't Hooft-Veltman procedure [15] is assumed to be used when it is necessary. Thus, the complete one-loop divergences for the theory (5) become

$$
\begin{aligned}
& \Gamma_{d i v}=-\frac{1}{2 \epsilon} \int d^{2} x \sqrt{g}\left\{\frac{48+2 m}{12} R+\frac{2}{C} V+\frac{2}{C^{\prime}} V^{\prime}-\frac{V_{, i i}}{f}\right. \\
& \left.+\left(\frac{C^{\prime \prime}}{C}-\frac{3 C^{\prime 2}}{C^{2}}-\frac{C^{\prime \prime} Z}{C^{\prime 2}}-\frac{m f^{\prime 2}}{4 f^{2}}+\frac{m f^{\prime \prime}}{2 f}\right)\left(\nabla^{\lambda} \Phi\right)\left(\nabla_{\lambda} \Phi\right)+\left(\frac{C^{\prime}}{C}-\frac{Z}{C^{\prime}}+\frac{m f^{\prime}}{2 f}\right) \Delta \Phi\right\} .
\end{aligned}
$$

This is the main result of the present section - the one-loop effective action for $2 \mathrm{~d}$ dilaton gravity with scalar matter. Furthermore, one can easily generalize this expression to the case when Maxwell fields are added, namely, when one considers action (1) plus the Maxwell action:

$$
S=-\int d^{2} x \sqrt{g}\left[\cdots+\frac{1}{4} f_{1}(\Phi) F_{\mu \nu}^{2}\right] .
$$

With the background versus quantum field separation $A_{\mu} \rightarrow A_{\mu}+Q_{\mu}$, in the Lorentz gauge,

$$
S_{\text {Lorentz }}=-\int d^{2} x \sqrt{g} f_{1}(\Phi)\left(\nabla_{\mu} Q_{\mu}\right)^{2},
$$

the extra contributions are known to split into the $F^{2}$-terms and total divergences (see ref. [16]). Thus, the total divergent contribution to the one-loop effective action of the theory (5) plus the Maxwell terms (33) is given by

$$
\begin{aligned}
& \Gamma_{d i v}=-\frac{1}{2 \epsilon} \int d^{2} x \sqrt{g}\left\{\frac{-2 m+60}{12} R+\frac{2}{C} V\right.+\frac{2}{C^{\prime}} V^{\prime}-\frac{V_{, i i}}{f}+\left(\frac{f_{1}{ }^{\prime}}{2 C^{\prime}}-\frac{f_{1}}{2 C}\right) F_{\mu \nu}^{2} \\
&+\left(\frac{C^{\prime \prime}}{C}-\frac{3 C^{\prime 2}}{C^{2}}-\frac{C^{\prime \prime} Z}{C^{\prime 2}}-\frac{m f^{\prime 2}}{4 f^{2}}+\frac{m f^{\prime \prime}}{2 f}+\frac{f_{1}^{\prime \prime}}{f_{1}}-\frac{f_{1}^{\prime 2}}{f_{1}^{2}}\right)\left(\nabla^{\lambda} \Phi\right)\left(\nabla_{\lambda} \Phi\right) \\
&\left.+\left(\frac{C^{\prime}}{C}-\frac{Z}{C^{\prime}}+\frac{m f^{\prime}}{2 f}+\frac{f_{1}{ }^{\prime}}{f_{1}}\right) \Delta \Phi\right\}
\end{aligned}
$$


This gives the divergences of the covariant effective action (general review is given in [2]). Gauge dependence of Eq.(35) has been studied in ref. 17. It is interesting to note that such one-loop EA may be also calculated in gauge-fixing condition independent formulation [17.

In the similar way, one can calculate the one-loop effective action for the theory (5) in the case when the gravitational field is a classical one, but the dilaton and the scalar fields are quantized. Such a calculation is much simpler since there are no gauge-fixing terms and corresponding ghosts. The effective action may be found as following

$$
\begin{aligned}
& \Gamma_{d i v}=-\frac{1}{2 \epsilon} \int d^{2} x \sqrt{g}\left\{\left(\frac{C^{\prime \prime}}{Z}-\frac{2 m+2}{12}\right) R+\frac{V^{\prime \prime}}{Z}-\frac{V_{, i i}}{f}+\left(\frac{f^{\prime 2}}{2 f Z}-\frac{f^{\prime \prime}}{2 Z}\right)\left(\nabla^{\lambda} \chi\right)\left(\nabla_{\lambda} \chi\right)\right. \\
& \left.+\left(\frac{m f^{\prime \prime}}{2 f}-\frac{m f^{\prime 2}}{4 f^{2}}-\frac{Z^{\prime 2}}{4 Z^{2}}\right)\left(\nabla^{\lambda} \Phi\right)\left(\nabla_{\lambda} \Phi\right)+\left(\frac{m f^{\prime}}{2 f}-\frac{Z^{\prime}}{2 Z}\right) \Delta \Phi\right\} .
\end{aligned}
$$

Expression (36) gives the one-loop effective action of the system composed of quantized dilaton, scalars in an external classical gravitational field.

Let us make here remarks on the issue of renormalization, to one-loop order. Without loss of generality, we will restrict ourselves to the case of pure $2 \mathrm{~d}$ dilaton gravity. By adding to the classical action the corresponding counterterms ( $\Gamma_{\text {div }}$ with opposite sign), one obtains the one-loop renormalized effective action.

Choosing the renormalization of the metric tensor in the following form

$$
g_{\mu \nu}=\exp \left\{\frac{1}{\epsilon}\left[\frac{1}{C(\Phi)}+\frac{Z(\Phi)}{2 C^{\prime 2}(\Phi)}\right]\right\} g_{\mu \nu}^{R}
$$

(this choice absorbs all the divergences of the dilaton kinetic term), one can obtain the renormalized effective action as (for simplicity, we drop the superscript ' $\mathrm{R}$ ' off $g_{\mu \nu}$ and $R$ ):

$$
S_{R}=-\int d^{2} x \sqrt{g}\left\{\frac{1}{2} Z g^{\mu \nu} \partial_{\mu} \Phi \partial_{\nu} \Phi+C R+V+\frac{1}{\epsilon}\left(-\frac{V^{\prime}}{C^{\prime}}+\frac{Z V}{2 C^{\prime 2}}\right)\right\} .
$$

Now, the condition of multiplicative renormalizability of the theory in the usual sense has the following form

$$
-\frac{V^{\prime}}{C^{\prime}}+\frac{Z V}{2 C^{\prime 2}}=a_{1} V
$$

where $a_{1}$ is an arbitrary constant. This condition restricts the form of the functions under discussion. Some sets of solutions of Eqs. (39) can be obtained explicitly:

$$
Z=1, \quad C(\Phi)=C_{1} \Phi, \quad V=0 .
$$

There are, of course, more complicated solutions [4, 5, 6]. One can also look to fixed points solutions of generalized RG equations, finding some specific models of dilatonic gravity as fixed points of such RG equations [17.

\subsection{Quantum equivalence in the models of $2 \mathrm{~d}$ dilatonic gravity}

Let us limit to specific version of $2 \mathrm{~d}$ dilatonic gravity with action:

$$
S_{1}=-\int d^{2} x \sqrt{g}\left[\frac{1}{2} g^{\mu \nu} \partial_{\mu} \Phi \partial_{\nu} \Phi+C R \Phi+V(\Phi)\right],
$$


where $C$ is a positive constant and $V(\Phi)$ an arbitrary function. The one-loop renormalization of the theory (41) has been discussed above. We will study quantum equivalence in such model following to ref. 18 .

Let us here briefly summarize the results concerning the one-loop effective action. We use the background field method

$$
g_{\mu \nu} \longrightarrow \bar{g}_{\mu \nu}=g_{\mu \nu}+h_{\mu \nu}, \quad \Phi \longrightarrow \bar{\Phi}=\Phi+\varphi,
$$

where $h_{\mu \nu}$ and $\varphi$ are the quantum fields. The simplest minimal covariant gauge is given by

$$
S_{G F}=-\frac{1}{2} \int d^{2} x c_{\mu \nu} \chi^{\mu} \chi^{\nu}
$$

where

$$
c_{\mu \nu}=-C \Phi \sqrt{g} g_{\mu \nu}, \quad \chi^{\mu}=-\nabla^{\nu} \bar{h}^{\mu}{ }_{\nu}+\frac{1}{\Phi} \nabla^{\mu} \varphi
$$

and $\bar{h}_{\mu \nu}=h_{\mu \nu}-\frac{1}{2} g_{\mu \nu} h$. The divergences of the one-loop effective action (including all surface terms) have been calculated in Eq.(31) as following:

$$
\Gamma_{d i v}=-\frac{1}{2 \epsilon} \int d^{2} x \sqrt{g}\left[4 R+\frac{2}{C \Phi} V+\frac{2}{C} V^{\prime}+\left(\frac{1}{\Phi}-\frac{1}{C}\right)(\Delta \Phi)-\frac{3}{\Phi^{2}}\left(\nabla^{\lambda} \Phi\right)\left(\nabla_{\lambda} \Phi\right)\right] .
$$

Let us now make in the theory (41) the field transformation

$$
\Psi^{2}=\frac{C}{\gamma} \Phi, \quad g_{\mu \nu} \longrightarrow e^{-2 \rho} \widetilde{g}_{\mu \nu}
$$

where

$$
\gamma>0, \quad \rho=\frac{\gamma \Psi^{2}}{4 C^{2}}-\frac{1}{8 \gamma} \ln \Psi
$$

Then, action (41) becomes

$$
S_{2}=-\int d^{2} x \sqrt{\widetilde{g}}\left[\frac{1}{2} \widetilde{g}^{\mu \nu} \partial_{\mu} \Psi \partial_{\nu} \Psi+\gamma \widetilde{R} \Psi^{2}+U(\Psi)\right],
$$

where we have defined $U(\Psi) \equiv \mathrm{e}^{-2 \rho} V(\Phi(\Psi))$ and dropped off a total derivative term. The actions $S_{1}$ and $S_{2}$ (eqs. (41) and (47), respectively) are parameterized through different triplets of functions $\{Z, C, V\}$. They are classically equivalent and lead to the same classical physics.

We shall now investigate the one-loop effective action for the theory (47). The calculation will be done in the same gauge (43)-(44), that is also to be transformed in accordance with (46). So, the natural prescription is:

a). For the background fields we shall make the transformation (46), where $\Psi$ and $\widetilde{g}_{\mu \nu}$ will be now the background fields of the theory (47).

b). The quantum fields will be transformed according to the first order Taylor expansion of eq. 46. (local change of variables)

$$
\varphi \longrightarrow \frac{2 \gamma}{C} \Psi \eta, \quad h_{\mu \nu} \longrightarrow \mathrm{e}^{-2 \rho(\Psi)}\left[\widetilde{h}_{\mu \nu}+\left(\frac{1}{4 \gamma \Psi}-\frac{\gamma}{C^{2}} \Psi\right) \widetilde{g}_{\mu \nu} \eta\right]
$$

We should recall now that in the background field method for the theory (47),

$$
\widetilde{g}_{\mu \nu} \longrightarrow \widetilde{g}_{\mu \nu}+\widetilde{h}_{\mu \nu}, \quad \Psi \longrightarrow \Psi+\eta
$$


where $\widetilde{h}_{\mu \nu}$ and $\eta$ are the quantum fields. Taking into account all the remarks above, we get the following covariant (minimal) gauge for the theory (47):

$$
S_{G F}=-\frac{1}{2} \int d^{2} x c_{\mu \nu} \chi^{\mu} \chi^{\nu},
$$

where

$$
\begin{aligned}
c_{\mu \nu} & =-\gamma \Psi^{2} \sqrt{g} g_{\mu \nu}, \\
\chi^{\mu} & =-\nabla^{\nu} \bar{h}_{\mu \nu}+\left(\frac{\gamma}{C^{2}} \Psi-\frac{1}{4 \gamma \Psi}\right)\left(\nabla^{\nu} \Psi\right) \bar{h}^{\mu}{ }_{\nu}+\frac{2}{\Psi} \nabla^{\mu} \eta+\frac{2}{\Psi^{2}}\left(\nabla^{\mu} \Psi\right) \eta .
\end{aligned}
$$

In order to simplify notation, in which follows we can suppress tildes over $g_{\mu \nu}$ and $h_{\mu \nu}$. The total one-loop divergence, $\Gamma_{d i v}=\Gamma_{2-d i v}+\Gamma_{g h o s t-d i v}$, is

$$
\begin{aligned}
\Gamma_{d i v} & =-\frac{1}{2 \epsilon} \int d^{2} x \sqrt{g}\left[4 R+\frac{2}{\gamma \Psi^{2}} U+\frac{1}{\gamma \Psi} U^{\prime}+\left(\frac{4 \gamma-3}{2 \gamma \Psi}+\frac{4 \gamma \Psi}{C^{2}}\right)(\Delta \Psi)\right. \\
& \left.+\left(\frac{4 \gamma}{C^{2}}+\frac{1-20 \gamma}{2 \gamma \Psi^{2}}\right)\left(\nabla^{\lambda} \Psi\right)\left(\nabla_{\lambda} \Psi\right)\right] .
\end{aligned}
$$

We must observe that in this final formula all surface terms have been kept.

Let us now discuss the on-shell limit of $\Gamma_{d i v}$, eq. (53). Keeping all the surface terms and using the classical field equations resulting from the action (47) we obtain the total derivative

$$
\Gamma_{d i v}^{o n-s h e l l}=-\frac{1}{2 \epsilon} \int d^{2} x \sqrt{g}\left[2 R+\Delta\left(\frac{12 \gamma-1}{2 \gamma} \ln \Psi+\frac{2 \gamma}{C^{2}} \Psi^{2}\right)\right] .
$$

One may consider another form of gauge fixing action. Then, one-loop effective action in new gauge differs from $\Gamma_{d i v}$, eq. (53), only in surface terms. The off-shell one-loop renormalization is the same in different gauges. However, even on shell they still differ in some total derivative terms. Summing up, we see that the on-shell effective action in different gauges is given by surface divergences only (finiteness of the $S$ matrix), but these terms depend yet on the choice of gauge condition.

We are now going to investigate the theory (41) in the variables (46). Transforming $\Gamma_{d i v}$, eq. (45), to the new variables (46), we get

$$
\begin{aligned}
\Gamma_{d i v} & =-\frac{1}{2 \epsilon} \int d^{2} x \sqrt{g}\left[4 R+\frac{2}{\gamma \Psi^{2}} U+e^{-2 \rho(\Psi)} \frac{2}{\gamma} \frac{\partial V(\Psi)}{\partial \Psi}\right. \\
& \left.+\left(\frac{4 \gamma-2}{2 \gamma \Psi}+\frac{2 \gamma \Psi}{C^{2}}\right) \Delta \Psi+\left(\frac{2 \gamma}{C^{2}}+\frac{2-20 \gamma}{2 \gamma \Psi^{2}}\right)\left(\nabla^{\lambda} \Psi\right)\left(\nabla_{\lambda} \Psi\right)\right],
\end{aligned}
$$

As we see, there is no perturbative quantum equivalence between the two classically equivalent dilaton gravities (41) and (47). In fact, the one-loop effective action (55) which comes from action (41) in the gauge (43) does not coincide with $\Gamma_{d i v}$, eq. (53), which is obtained when starting from the classically equivalent theory (47) in the gauge (50) - that can be made correspond with the gauge (43). However one can show that two classically equivalent dilaton gravities lead to the same class of multiplicatively renormalizable potentials.

Let us now consider the on-shell effective action of the theory (41). Using classical field equations and keeping all the total derivative terms in (45), we get

$$
\Gamma_{\text {div }}^{\text {on-shell }}=-\frac{1}{2 \epsilon} \int d^{2} x \sqrt{g}\left[2 R+\Delta\left(\frac{1}{C} \Phi+3 \ln \Phi\right)\right] .
$$


Transforming the variables in (56) according to the change (46), we obtain exactly eq. (54).

From the discussion above, we conclude that there is on-shell perturbative quantum equivalence for the classically equivalent dilaton gravities (41) and (47). This analysis is presented in detail in order to avoid the following confusion. It is very often to study some questions related with quantum cosmology or black holes the researchers come to "convenient" parameterization of the quantum model where quantum calculations are easier to do. After that, the result of such calculations is transformed back to original parameterization (original quantum variables) and is used in subsequent analysis. As it follows from above results such approach is most likely wrong unless on-shell calculations are used.

\subsection{Dilatonic gravity with matter in $2+\epsilon$-dimensions}

It is quite an old idea [19, 20, 21, 22, 23, to try to study Einstein gravity in $2+\epsilon$ dimensions, which may improve its ultraviolet properties. The gravitational coupling constant in such a theory - near two dimensions - shows an asymptotically free behavior [19, 20, 21, 22, 23], which can actually be very exciting in attempts to solve the problem of non-renormalizability of 4-dimensional Einstein gravity [15]. Unfortunately, it was shown that Einstein gravity in $2+\epsilon$ dimensions is on its turn a non-renormalizable theory [24] in the usual sense.

Furthermore, dynamical triangulations in more than two dimensions (see, for example, [25, 26]) have clearly shown the existence of a phase transition to a strong-coupling phase of similar nature as 2dimensional quantum gravity [27]. Because of this, of considerable interest is still to try to construct a consistent theory of QG in $2+\epsilon$ dimensions (on the next step, one can think on hypothetical continuation of small $\epsilon$ to $\epsilon=1$ or $\epsilon=2$ ).

It has been suggested [28], that a thing to do would be to study dilatonic gravity near two dimensions (similarly, it was the idea in ref. [29] to consider Einstein gravity with a conformal scalar field near two dimensions). Notice, however, that unlike Einstein gravity, dilatonic gravity possesses a smooth $\epsilon \rightarrow 0$ limit. This difference manifests itself in the fact that dilatonic gravity - which after a proper field definition can be presented as an Einsteinian theory with a scalar field - is not equivalent to Einstein gravity in exactly two dimensions. The gravitational coupling constant 28] in this theory also has an ultraviolet stable fixed point for $\epsilon>0$ and $n<24$ ( $n$ is the number of scalars or matter central charge). Thus, the matter central charge in dilatonic gravity is bounded, as it also happens in Einstein's theory.

In the present section, we give short overview of typical example: dilatonic-Maxwell gravity with scalar matter in $2+\epsilon$ dimensions. This theory, which includes a dilatonic potential and a Maxwell term with an arbitrary dilatonic-vector coupling function, may be considered as a toy model for unification

of gravity with matter (scalar and vector fields). We follow to ref. [30, 31] where it was shown that in the ultraviolet stable fixed point the gravitational coupling constant has the value $G^{*}=3 \epsilon /[2(30-n)]$ and, hence, owing to the contribution of the vectors, the matter central charge of our universe in such a model can be naturally increased from $0<n<24$ (for pure dilatonic gravity) to $0<n<30$ (for dilatonic-Maxwell gravity).

We start from the theory of dilatonic gravity interacting with scalars and vectors via dilatonic couplings 
in $2+\epsilon$ dimensions. The action is

$$
S=\int d^{d} x \sqrt{-g}\left[\frac{1}{2} Z(\phi) g^{\mu \nu} \partial_{\mu} \phi \partial_{\nu} \phi+\frac{\mu^{\epsilon}}{16 \pi G} R C(\phi)+V(\phi)-\frac{1}{2} f(\phi) g^{\mu \nu} \partial_{\mu} \chi_{i} \partial_{\nu} \chi^{i}+\frac{1}{4} f_{1}(\phi) F_{\mu \nu}^{2}\right],
$$

where $g_{\mu \nu}$ is the $(2+\epsilon)$-dimensional metric, $R$ the corresponding curvature, $\chi_{i}$ are scalars $(i=1,2, \ldots, n)$, $F_{\mu \nu}=\nabla_{\mu} A_{\nu}-\nabla_{\nu} A_{\mu}$, with $A_{\mu}$ a vector, and where the smooth functions $Z(\phi), C(\phi), V(\phi), f(\phi)$ and $f_{1}(\phi)$ describe the dilatonic interactions. Notice that $V(\phi)$ is a dimensional function and it is therefore convenient to redefine $V \rightarrow m^{2} V$, where $m$ is some parameter with dimension of mass.

One can easily prove that the above theory (57) is renormalizable in a generalized sense. The one-loop counterterms corresponding to (57) in two dimensions have been calculated in refs. [14, 16], (see also previous section). Our purpose is to present the renormalization structure of (57) in $2+\epsilon$ dimensions and in particular the corresponding renormalization group equations. The very remarkable point that gives sense to this study is the fact that the theory (57) (unlike Einstein gravity [19, 20, 21, 22, 23]) has a smooth limit for $\epsilon \rightarrow 0$. This property allows for the possibility to study the behavior of (57) in $2+\epsilon$ dimensions by simply using the counterterms calculated already in 2 dimensions - in close analogy with quantum field theory in frames of the $\epsilon$-expansion technique (for a review see [32] and [33, 34]).

Action (57) may be rewritten in another form. First, motivated by the form of the string effective action, we choose the dilatonic couplings in (57) as the exponents of convenient dilatonic functions. Using the rigid rescaling of scalars and vectors:

$$
\chi_{i} \longrightarrow a_{1} \chi_{i}, \quad A_{\mu} \longrightarrow a_{2} A_{\mu},
$$

with $a_{1}$ and $a_{2}$ constants, one can always normalize the dilatonic couplings in such a manner that

$$
f(0)=1, \quad f_{1}(0)=1 .
$$

Second, as has been done in refs. [10, 6], we can also use a local Weyl transformation of the metric of the form

$$
g_{\mu \nu} \longrightarrow \mathrm{e}^{-2 \sigma(\phi)} g_{\mu \nu},
$$

in order to simplify the dilatonic sector of (57). In particular, one can use (60) of a specific form such that $Z(\phi)=0$. Finally, we can perform a transformation of the dilaton field in order to simplify the function $C(\phi)$, namely to reduce it to the form $C(\phi)=e^{-2 \phi}$. With all this taken into account, the action (57) can be written as follows

$$
S=\int d^{d} x \sqrt{-g}\left[\frac{\mu^{\epsilon}}{16 \pi G} R \mathrm{e}^{-2 \phi}-\frac{1}{2} g^{\mu \nu} \partial_{\mu} \chi_{i} \partial_{\nu} \chi^{i} \mathrm{e}^{-2 \Phi(\phi)}+\mu^{\epsilon} m^{2} \mathrm{e}^{-V(\phi)}+\frac{1}{4} \mathrm{e}^{-f_{2}(\phi)} F_{\mu \nu}^{2}\right],
$$

being $\mu$ a mass parameter and where we have chosen the dilatonic couplings so that $\Phi(0)=f_{2}(0)=$ $V(0)=0$. The first two terms in (61) correspond to the dilatonic gravity action in ref. [28], which was considered in $2+\epsilon$ dimensions. With our choice of (61) the zero modes of the dilatonic couplings are fixed with the help of reference operators in the gravitational sector [28] and with the renormalization (by constants) of the scalar, vector, and mass $m^{2}$ in the matter sector.

We can now present short resume of the divergences of the action (61). The study of the one-loop divergences of dilatonic gravity in the covariant formalism, initiated in refs. [4, 河, has been continued in 
refs. 10, 6, 14, 11. We do not consider it necessary to repeat the details of such a calculation for the case of the model (61), see for more details, the previous section. The gauge-fixing conditions that are most convenient to use are the following. The covariant gauge-fixing action is

$$
S_{g f}=-\frac{\mu^{\epsilon}}{32 \pi G} \int d^{d} x \sqrt{-g} g_{\mu \nu} \chi^{\mu} \chi^{\nu} \mathrm{e}^{-2 \phi}
$$

where

$$
\chi^{\mu}=\nabla_{\nu} \bar{h}^{\mu \nu}+2 \nabla^{\mu} \varphi
$$

and $\bar{h}^{\mu \nu}$ is a traceless quantum gravitational field and $\varphi$ a quantum scalar field, in the background field method. In the electromagnetic sector the gauge-fixing action is chosen as

$$
S_{L}=\int d^{d} x \sqrt{-g}\left(\nabla_{\mu} Q^{\mu}\right)^{2} \mathrm{e}^{-f_{2}(\phi)},
$$

where $Q_{\mu}$ is a quantum vector field. Notice that for the background fields we use the same notations as for the classical fields in (61).

The calculation of the one-loop effective action corresponding to (61) in the gauges (62) and (64) can be performed in close analogy with the one in refs. 14, 16. This is reviewed in previous section. Owing to the smooth behavior of (61) for $\epsilon \rightarrow 0$, the divergences of (61) can be also calculated in exactly two dimensions, which will also provide the result for $2+\epsilon$ dimensions:

$$
\begin{aligned}
\Gamma_{d i v}= & \frac{1}{4 \pi \epsilon} \int d^{d} x \sqrt{-g}\left\{\frac{30-n}{6} R+m^{2} \mathrm{e}^{2 \phi-V(\phi)} 16 \pi G\left[2+V^{\prime}(\phi)\right]\right. \\
& \left.-g^{\mu \nu} \partial_{\mu} \phi \partial_{\nu} \phi\left[8-n \Phi^{\prime}(\phi)^{2}\right]+\frac{4 \pi G}{\mu^{\epsilon}} \mathrm{e}^{2 \phi-f_{2}(\phi)} F_{\mu \nu}^{2}\left[f_{2}^{\prime}(\phi)-2\right]\right\},
\end{aligned}
$$

where $\epsilon=d-2$. As usually off-shell it is gauge dependent.

We turn to the study of the RG corresponding to our model in $2+\epsilon$ dimensions. The main idea of the whole approach is to use $G$ as the coupling constant in perturbation theory and work at some fixed power of $G$. The counterterm follows from (65)

$$
\Gamma_{\text {count }}=-\mu^{\epsilon} \int d^{d} x \sqrt{-g}\left[R A_{1}(\phi)+g^{\mu \nu} \partial_{\mu} \phi \partial_{\nu} \phi A_{2}(\phi)+m^{2} A_{3}(\phi)+\frac{\mu^{-\epsilon}}{4} F_{\mu \nu}^{2} A_{4}(\phi)\right],
$$

where

$$
\begin{aligned}
& A_{1}(\phi)=\frac{30-n}{24 \pi \epsilon} \equiv A_{1}, \quad A_{2}(\phi)=\frac{1}{4 \pi \epsilon}\left[n \Phi^{\prime}(\phi)^{2}-8\right], \\
& A_{3}(\phi)=\frac{4 G}{\epsilon}\left[2+V^{\prime}(\phi)\right] \mathrm{e}^{2 \phi-V(\phi)} \equiv \mathrm{e}^{-V(\phi)} \widetilde{A}_{3}(\phi), \\
& A_{4}(\phi)=\frac{4 G}{\epsilon}\left[f_{2}^{\prime}(\phi)-2\right] \mathrm{e}^{2 \phi-f_{2}(\phi)} \equiv \mathrm{e}^{-f_{2}(\phi)} \widetilde{A}_{4}(\phi) .
\end{aligned}
$$

Renormalization is now performed in the standard way

$$
\begin{aligned}
S= & S_{c l}+S_{\text {count }}=\int d^{d} x \sqrt{-g}\left[\frac{1}{16 \pi G_{0}} R \mathrm{e}^{-2 \phi_{0}}-\frac{1}{2} g_{0}^{\mu \nu} \partial_{\mu} \chi_{0 i} \partial_{\nu} \chi_{0}^{i} \mathrm{e}^{-2 \Phi_{0}\left(\phi_{0}\right)}\right. \\
& \left.+\mu^{\epsilon} m_{0}^{2} \mathrm{e}^{-V_{0}\left(\phi_{0}\right)}+\frac{1}{4} \mathrm{e}^{-f_{02}\left(\phi_{0}\right)} g_{0}^{\mu \alpha} g_{0}^{\nu \beta} F_{0 \mu \nu} F_{0 \alpha \beta}\right]
\end{aligned}
$$


The renormalization transformations can be defined as follows

$$
\begin{aligned}
& \phi_{0}=\phi+f(\phi), \quad g_{0 \mu \nu}=g_{\mu \nu} e^{-2 \Lambda(\phi)}, \quad \Phi_{0}\left(\phi_{0}\right)=\Phi(\phi)+F(\phi), \quad V_{0}\left(\phi_{0}\right)=V(\phi)+F_{V}(\phi), \\
& f_{02}\left(\phi_{0}\right)=f_{2}(\phi)+F_{f}(\phi), \quad \chi_{0 i}=Z_{\chi}^{1 / 2} \chi, \quad A_{0 \mu}=Z_{A}^{1 / 2} A_{\mu}, \quad m_{0}^{2}=Z_{m} m^{2} .
\end{aligned}
$$

The functions $\Lambda, f, F, \ldots$ are chosen so that $\Lambda(0), f(0), F(0), \ldots$ can be set equal to zero. Substituting the renormalization transformations (69) into the renormalized action (68) (in close analogy with ref. 286), we obtain

$$
\begin{aligned}
S_{0}= & \int d^{d} x \sqrt{-g}\left\{\frac{1}{16 \pi G_{0}} R e^{-2 \phi-2 f(\phi)-\epsilon \Lambda(\phi)}\right. \\
& +\frac{\epsilon+1}{16 \pi G_{0}}\left[4 \Lambda^{\prime}(\phi)+\epsilon \Lambda^{\prime}(\phi)^{2}+4 f^{\prime}(\phi) \Lambda^{\prime}(\phi)\right] e^{-2 \phi-2 f(\phi)-\epsilon \Lambda(\phi)} g^{\mu \nu} \partial_{\mu} \phi \partial_{\nu} \phi \\
& -\frac{1}{2} Z_{\chi} g^{\mu \nu} \partial_{\mu} \chi_{i} \partial_{\nu} \chi^{i} \mathrm{e}^{-2 \Phi(\phi)-2 F(\phi)-\epsilon \Lambda(\phi)}+\mu^{\epsilon} m^{2} Z_{m} \mathrm{e}^{-V(\phi)-F_{V}(\phi)-(2+\epsilon) \Lambda(\phi)} \\
& \left.+\frac{Z_{A}}{4} \mathrm{e}^{-f_{2}(\phi)-F_{f}(\phi)+(2-\epsilon) \Lambda(\phi)} F_{\mu \nu}^{2}\right\} .
\end{aligned}
$$

From here, one can easily obtain

$$
\begin{aligned}
& \frac{1}{16 \pi G_{0}} \mathrm{e}^{-2 \phi-2 f(\phi)-\epsilon \Lambda(\phi)}=\mu^{\epsilon}\left(\frac{1}{16 \pi G} \mathrm{e}^{-2 \phi}-A_{1}\right), \\
& \frac{\epsilon+1}{16 \pi G_{0}}\left[4 \Lambda^{\prime}(\phi)+\epsilon \Lambda^{\prime}(\phi)^{2}+4 \epsilon f^{\prime}(\phi) \Lambda^{\prime}(\phi)\right] e^{-2 \phi-2 f(\phi)-\epsilon \Lambda(\phi)}=-\mu^{\epsilon} A_{2}(\phi), \\
& Z_{\chi} \mathrm{e}^{-2 \Phi(\phi)-2 F(\phi)-\epsilon \Lambda(\phi)}=\mathrm{e}^{-2 \Phi(\phi)}, \quad Z_{A} \mathrm{e}^{-f_{2}(\phi)-F_{f}(\phi)+(2-\epsilon) \Lambda(\phi)}=\mathrm{e}^{-f_{2}(\phi)}\left[1-\widetilde{A}_{4}(\phi)\right], \\
& Z_{m} \mathrm{e}^{-V(\phi)-F_{V}(\phi)-(2+\epsilon) \Lambda(\phi)}=\mathrm{e}^{-V(\phi)}\left[1-\widetilde{A}_{3}(\phi)\right] .
\end{aligned}
$$

Working to leading order in $G$ (notice that the functions $f, \Lambda, F, \ldots, F_{f}$ are of first order in $G$ ), we may follow ref. 28 and obtain the renormalized parameters as

$$
\begin{aligned}
& G_{0}^{-1}=\mu^{\epsilon}\left(G^{-1}-16 \pi A_{1}\right), \quad Z_{\chi}=1, \quad \Lambda(\phi)=-\frac{4 \pi G}{\epsilon+1} \int_{0}^{\phi} d \phi^{\prime} \mathrm{e}^{2 \phi^{\prime}} A_{2}\left(\phi^{\prime}\right), \quad \epsilon \Lambda(\phi)=-2 F(\phi), \\
& f(\phi)=8 \pi A_{1} G\left(e^{2 \phi}-1\right)+\frac{2 \pi \epsilon G}{\epsilon+1} \int_{0}^{\phi} d \phi^{\prime} \mathrm{e}^{2 \phi^{\prime}} A_{2}\left(\phi^{\prime}\right), \quad Z_{A}=1-A_{4}(0), \quad Z_{m}=1-A_{3}(0), \\
& F_{f}(\phi)=(2-\epsilon) \Lambda(\phi)+\widetilde{A}_{4}(\phi)-\widetilde{A}_{4}(0), \quad F_{V}(\phi)=-(2+\epsilon) \Lambda(\phi)+\widetilde{A}_{3}(\phi)-\widetilde{A}_{3}(0) .
\end{aligned}
$$

Hence, we see that the zero modes of the functions $f, f_{2}$ and $V$ are indeed controlled by a constant renormalization of the vector, the scalar and the mass.

We can now present the beta functions. We will list below only the ones corresponding to the dilatonic couplings and gravitational constant

$$
\begin{aligned}
& \beta_{G}=\mu \frac{\partial G}{\partial \mu}=\epsilon G-16 \pi \epsilon A_{1} G^{2}, \quad \mu \frac{\partial \Lambda\left(\phi_{0}\right)}{\partial \mu}=-\frac{4 \pi \epsilon G}{\epsilon+1} \int_{0}^{\phi} d \phi^{\prime} \mathrm{e}^{2 \phi^{\prime}} A_{2}\left(\phi^{\prime}\right), \\
& \beta_{\Phi}\left(\phi_{0}\right)=\mu \frac{\partial \Phi\left(\phi_{0}\right)}{\partial \mu}=8 \pi \epsilon A_{1} G\left(\mathrm{e}^{2 \phi_{0}}-1\right) \Phi^{\prime}\left(\phi_{0}\right)+\frac{2 \pi \epsilon^{2} G}{\epsilon+1}\left[\Phi^{\prime}\left(\phi_{0}\right)-1\right] \int_{0}^{\phi_{0}} d \phi^{\prime} \mathrm{e}^{2 \phi^{\prime}} A_{2}\left(\phi^{\prime}\right), \\
& \beta_{f_{2}}\left(\phi_{0}\right)=\mu \frac{\partial f_{2}\left(\phi_{0}\right)}{\partial \mu}=8 \pi \epsilon A_{1} G\left(\mathrm{e}^{2 \phi_{0}}-1\right) f_{2}^{\prime}\left(\phi_{0}\right) \\
& \quad+\frac{2 \pi \epsilon G}{\epsilon+1}\left[\epsilon f_{2}^{\prime}\left(\phi_{0}\right)+2(2-\epsilon)\right] \int_{0}^{\phi_{0}} d \phi^{\prime} \mathrm{e}^{2 \phi^{\prime}} A_{2}\left(\phi^{\prime}\right)-\epsilon\left[\widetilde{A}_{4}\left(\phi_{0}\right)-\widetilde{A}_{4}(0)\right],
\end{aligned}
$$




$$
\begin{aligned}
& \beta_{V}\left(\phi_{0}\right)=\mu \frac{\partial V\left(\phi_{0}\right)}{\partial \mu}=8 \pi \epsilon A_{1} G\left(e^{2 \phi_{0}}-1\right) V^{\prime}\left(\phi_{0}\right) \\
& +\frac{2 \pi \epsilon G}{\epsilon+1}\left[\epsilon V^{\prime}\left(\phi_{0}\right)-2(2+\epsilon)\right] \int_{0}^{\phi_{0}} d \phi^{\prime} \mathrm{e}^{2 \phi^{\prime}} A_{2}\left(\phi^{\prime}\right)-\epsilon\left[\widetilde{A}_{3}\left(\phi_{0}\right)-\widetilde{A}_{3}(0)\right] .
\end{aligned}
$$

Similarly, one can obtain the $\gamma$-functions for the fields and mass parameter, which are however of less importance, due to the fact that they correspond to non-essential couplings. Notice also that the only conformal mode of the gravitational field is renormalized in $2+\epsilon$ dimensions, which is quite well known [19, 20, 21, 22, 23, 28.

The $\beta$-function for the gravitational constant has the form (73)

$$
\beta_{G}=\epsilon G-\frac{2(30-n)}{3} G^{2} .
$$

Hence, for $n<30$ one obtains the infrared stable fixed point $G=0$. There is also an ultraviolet stable fixed point as in $19,20,21,22,23$

$$
G^{*}=\frac{3 \epsilon}{2(30-n)}
$$

The theory is UV-stable (asymptotically free at exactly two dimensions).

One can start the search for fixed-point solutions corresponding to the dilatonic couplings. There is following Ansatz:

$$
\Phi(\phi)=\lambda \phi, \quad f_{2}(\phi)=\lambda_{f} \phi, \quad V(\phi)=\lambda_{V} \phi
$$

In that case the $\beta$-functions are simplified:

$$
\begin{aligned}
& \beta_{\Phi}=G\left(e^{2 \phi}-1\right)\left[\frac{30-n}{3} \lambda+\frac{\epsilon}{4(1+\epsilon)}(\lambda-1)\left(n \lambda^{2}-8\right)\right], \\
& \beta_{f_{2}}=G\left(e^{2 \phi}-1\right)\left[\frac{30-n}{3} \lambda_{f}+\frac{n \lambda^{2}-8}{4(1+\epsilon)}\left(\epsilon \lambda_{f}+4-2 \epsilon\right)-4 \lambda_{f}+8\right], \\
& \beta_{V}=G\left(e^{2 \phi}-1\right)\left[\frac{30-n}{3} \lambda_{V}+\frac{n \lambda^{2}-8}{4(1+\epsilon)}\left(\epsilon \lambda_{V}-4-2 \epsilon\right)-4 \lambda_{V}-8\right] .
\end{aligned}
$$

Equating these $\beta$-functions to zero we easily get the fixed-point solutions:

$$
\begin{aligned}
& \lambda^{*}=-\frac{6 \epsilon}{30-n}+\mathcal{O}\left(\epsilon^{2}\right), \\
& \lambda_{f}^{*}=-\frac{36 \epsilon}{18-n}+\mathcal{O}\left(\epsilon^{2}\right), \quad \lambda_{V}^{*}=\frac{12 \epsilon}{18-n}+\mathcal{O}\left(\epsilon^{2}\right) .
\end{aligned}
$$

The fixed-point solutions (79) are of the same nature as solution (78). Notice, however, that the denominator in (79) is different from the denominator for the case of a purely dilatonic sector (78). For the existence of the solutions (79) a new limitation appears: $n \neq 18$. Under a discontinuous transition through the point $n=18$, the sign of the fixed points in (79) changes.

It is interesting to see in which way the model (61) can be rewritten at the fixed point. In particular, we will perform a Weyl rescaling (it is non-singular) in the manner suggested in ref. 28]

$$
g_{\mu \nu} \longrightarrow g_{\mu \nu} \exp \left(\frac{4 \lambda^{*}}{\epsilon} \phi\right) .
$$


Then, the classical action becomes

$$
\begin{aligned}
S= & \int d^{d} x \sqrt{-g}\left\{\frac{\mu^{\epsilon}}{16 \pi G^{*}} e^{-2\left(1-\lambda^{*}\right) \phi}\left[R-\frac{4(1+\epsilon)}{\epsilon} \lambda^{*}\left(2-\lambda^{*}\right)\right] g^{\mu \nu} \partial_{\mu} \phi \partial_{\nu} \phi\right. \\
& \left.-\frac{1}{2} g^{\mu \nu} \partial_{\mu} \chi_{i} \partial_{\nu} \chi^{i}+\mu^{\epsilon} m^{2} \mathrm{e}^{\left(2 \lambda^{*}+4 \lambda^{*} / \epsilon-\lambda_{V}^{*}\right) \phi}+\frac{1}{4} F_{\mu \nu}^{2} \mathrm{e}^{\left(2 \lambda^{*}-4 \lambda^{*} / \epsilon-\lambda_{f}^{*}\right) \phi}\right\} .
\end{aligned}
$$

As one can see from expression (81), without the electromagnetic sector and setting $m^{2}=0$, it describes the CGHS action 35 (at the limit $\epsilon \rightarrow 0$ it is finite and scalars become non-interacting with the dilaton). As it stands, action (81) is of a similar form as dilaton-Maxwell gravity [36, 37, 16] with the Liouville potential. Notice that such kind of dilaton-Maxwell gravity (which can also be considered as a charged string-inspired model [36, 37, 38]) admits charged black hole solutions with multiple horizons, being in this sense analogous to four or higher-dimensional Einstein-Maxwell theories [39, 40]. Different forms of dilatonic gravity can be easily obtained too, by transforming the metric and the dilaton.

One can study the stability of the fixed points (75), (78), (79), along the same lines as in ref. [28]. Such analysis may be found in ref.[30]. It shows that above fixed points may be stable along some RG trajectories. The gauge dependence analysis of above fixed points has been also done in ref. [30, 31]. It follows from this analysis that gravitational constant in UV fixed point is gauge independent.

In this section we discussed dilatonic-Maxwell gravity with matter near two dimensions. The nice properties of this theory are: (i) its renormalizability in $2+\epsilon$ dimensions, (ii) The fact that there is a non-trivial fixed-point solution for the dilatonic couplings and that, at the fixed point, the theory may be represented in the standard form of the string-inspired models. (iii) The increase of the upper limit for the matter central charge - due to the contribution of the vector field - from 24 (pure dilatonic gravity) or 25 (Einstein gravity) to 30. More realistic models (like dilaton-Yang-Mills gravity) may be considered in a similar way [30, 31], as well as supergravity models [41]. It is also interesting that such models can be formulated as string theory.

Note that $2+\epsilon$-approach to quantum gravity did not finally succeeded to solve completely renormalizability issue of the quantum gravity in the usual sense. As a result the interest to this approach is minor now, it awaits until some new and fresh ideas will appear.

\subsection{One-loop effective action in $4 \mathrm{~d}$ higher derivative dilatonic gravity}

In the present section we show how one can generalize the discussion of one-loop effective action for $4 \mathrm{~d}$ dilatonic gravity. Supposing that $4 \mathrm{~d}$ dilaton is still dimensionless we naturally come to higher derivative theory of sigma-model type which is again renormalizable in a generalized sense. The most general form of such action looks like

$$
\begin{gathered}
S=\int d^{4} x \sqrt{-g}\left\{b_{1}(\varphi)(\square \varphi)^{2}+b_{2}(\varphi)\left(\nabla_{\mu} \varphi\right)\left(\nabla^{\mu} \varphi\right) \square \varphi+b_{3}(\varphi)\left[\left(\nabla_{\mu} \varphi\right)\left(\nabla^{\mu} \varphi\right)\right]^{2}\right. \\
+b_{4}(\varphi)\left(\nabla_{\mu} \varphi\right)\left(\nabla^{\mu} \varphi\right)+b_{5}(\varphi)+c_{1}(\varphi) R\left(\nabla_{\mu} \varphi\right)\left(\nabla^{\mu} \varphi\right)+c_{2}(\varphi) R^{\mu \nu}\left(\nabla_{\mu} \varphi\right)\left(\nabla_{\nu} \varphi\right) \\
\left.+c_{3}(\varphi) R \square \varphi+a_{1}(\varphi) R_{\mu \nu \alpha \beta}^{2}+a_{2}(\varphi) R_{\mu \nu}^{2}+a_{3}(\varphi) R^{2}+a_{4}(\varphi) R\right\}+ \text { (s.t.), }
\end{gathered}
$$

where s.t. means 'surface terms'. All generalized coupling constants are dimensionless, except for $b_{4}, b_{5}$ and $a_{4}$, for which we have: $\left[b_{4}(\varphi)\right]=2,\left[b_{5}(\varphi)\right]=4,\left[a_{4}(\varphi)\right]=2$. 
It is interesting to notice that, at the classical level and for some particular choices of the generalized couplings, the action (82) may be viewed, in principle, as a superstring theory effective action - the only background fields being the gravitational field and the dilaton. The calculation of one-loop effective action may be done using similar algorithm as in $2 \mathrm{~d}$ case (only some technical details are a bit more involved). Considering only case when dilaton is quantized and gravity is classical, one can get 42]

$$
\begin{gathered}
\Gamma_{\text {div }}^{(1-\text { loop })}=-\frac{2}{\varepsilon} \int d^{4} x \sqrt{-g}\left[A_{1} R_{\alpha \beta \gamma \tau}^{2}+A_{2} R_{\alpha \beta}^{2}+A_{3} R^{2}+A_{4} R m^{2}\right. \\
+C_{1} R\left(\nabla_{\mu} \varphi\right)^{2}+C_{2} R_{\mu \nu}\left(\nabla^{\mu} \varphi\right)\left(\nabla^{\nu} \varphi\right)+C_{3} R(\square \varphi) \\
\left.+B_{1}(\square \varphi)^{2}+B_{2}\left(\nabla_{\mu} \varphi\right)^{2}(\square \varphi)+B_{3}\left(\nabla_{\mu} \varphi\right)^{4}+B_{4} m^{2}\left(\nabla_{\mu} \varphi\right)^{2}+B_{5} m^{4}\right],
\end{gathered}
$$

where functions $A_{1}, \cdots, B_{5}$ are explicitly given in ref. 442].

Since the theory is renormalizable, one can formulate the renormalization group equations for the effective action and couplings and then explore its asymptotic behavior. The renormalization group equations for the effective action have the standard form, since the number (finite or infinite) of coupling constants is not essential. The general solution of this RG equation has the form

$$
\Gamma\left[e^{-2 t} g_{\alpha \beta}, a_{i}, b_{j}, c_{k}, \mu\right]=\Gamma\left[g_{\alpha \beta}, a_{i}(t), b_{j}(t), c_{k}(t), \mu\right]
$$

where $\mu$ is the renormalization parameter and the effective couplings satisfy renormalization group equations of the form

$$
\frac{d a_{i}(t)}{d t}=\beta_{a_{i}}, \quad a_{i}=a_{i}(0), \quad \frac{d b_{i}(t)}{d t}=\beta_{b_{i}}, \quad b_{i}=b_{i}(0), \quad \frac{d c_{i}(t)}{d t}=\beta_{c_{i}}, \quad c_{i}=c_{i}(0) .
$$

Note that we do not take into account the dimensions of the functions $a_{4}, b_{4}, b_{5}$. The derivation of the $\beta$-functions is pretty the same as in theories with finite number of couplings, and we easily get

$$
\beta_{a_{i}}=-(4 \pi)^{-2} A_{i}, \quad \beta_{b_{i}}=-(4 \pi)^{-2} B_{i}, \quad \beta_{c_{i}}=-(4 \pi)^{-2} C_{i} .
$$

The analysis of corresponding RG equations may be found in ref. 42 .

As an example, one can consider the most general conformally-invariant version of the theory (82):

$$
S_{c}=\int d^{4} x \sqrt{-g}\left\{f(\varphi) \varphi \nabla^{4} \varphi+q(\varphi) C_{\mu \nu \alpha \beta}^{2}+p(\varphi)\left[\left(\nabla_{\mu} \varphi\right)\left(\nabla^{\mu} \varphi\right)\right]^{2}\right\} .
$$

Here $f(\varphi), q(\varphi)$ and $p(\varphi)$ are arbitrary functions, $\nabla^{4}=\square^{2}+2 R^{\mu \nu} \nabla_{\mu} \nabla_{\nu}-\frac{2}{3} R \square+\frac{1}{3}\left(\nabla^{\mu} R\right) \nabla_{\mu}$ is a fourthorder conformally invariant operator, and we should recall that due to the fact that $[\varphi]=0$ the conformal transformation of our $4 \mathrm{~d}$ dilaton is trivial. One can integrate by parts the rhs in (87) and present the result as a particular case of the theory 82. As one can show, the divergences of the conformally-invariant theory (87) appear also in a conformally invariant form (up to the total divergence), as it should be. The functions $A(\varphi), B(\varphi), C(\varphi)$ obey the same conformal constraints with some $F(\varphi), Q(\varphi), P(\varphi)$ instead of $f(\varphi), q(\varphi), p(\varphi)$. Hence, the conformal invariant, higher-derivative scalar theory considered here is renormalizable at the one-loop level in a conformally invariant way, and therefore it is multiplicatively renormalizable at one-loop. 
Using explicit one-loop effective action one can search for finite and anomaly free models (via condition that one-loop EA is zero). One can also study renormalization group equations: theory under consideration possesses finite fixed points and interesting flows between these fixed points 42 . We do not give this analysis here (while it may have some applications for construction of infrared sector of quantum gravity [43, 44, 45]). Thus, to conclude one sees that perturbative renormalization of $4 \mathrm{~d}$ dilatonic gravity is possible but its detailed analysis looks much more complicated than $2 \mathrm{~d}$ case.

In summary, in this chapter we discussed the questions related with the calculation of one-loop effective action in $2 \mathrm{~d}$ and $4 \mathrm{~d}$ dilatonic gravity. It is also applied to study the model under discussion near two dimensions via epsilon-expansion. One can also consider applications of these effective actions for cosmology and observable consequences of gravity, like dilatonic contributions to Newton potential, construction of non-singular Universes (see chapter 5), etc.

\section{Conformal anomaly and induced effective action for dilaton coupled theories}

In the present chapter we will discuss in detail conformal anomaly and induced EA for dilaton coupled theories in two and four dimensions. The remarkable property of such EA is the fact that it will be given for arbitrary dilaton-gravitational background. As a result, it will be possible to apply it in different problems of BH physics and early Universe.

\subsection{2d dilaton coupled scalar and quantum dilaton: conformal anomaly and induced action}

In this section following to refs. 46, 47, we show how to get conformal anomaly and anomaly induced EA for $2 \mathrm{~d}$ dilaton coupled scalar with the help of results reported in second chapter.

Let us start from the following action:

$$
S=\int d^{2} x \sqrt{g}\left\{-\frac{1}{2} Z(\phi) g^{\mu \nu} \partial_{\mu} \phi \partial_{\nu} \phi+\frac{1}{2} f(\phi) g^{\mu \nu} \partial_{\mu} \chi_{i} \partial_{\nu} \chi_{i}\right\}
$$

where $\phi$ is dilaton, $Z, f$ are the arbitrary dilaton functions, $\chi$ is scalar field, $i=1, \cdots, N$. Here dilaton and scalars are quantum fields and gravitational field is considered to be external field. It is easy to show that such theory is conformally invariant in two dimensions.

We work with non-zero background dilaton and non-zero background scalar. The calculation of the one-loop effective action for such system has been done in all details in previous chapter. The result looks as follows (36) (see ref.[14):

$$
\begin{aligned}
\Gamma_{\text {div }}= & -\frac{1}{4 \pi(n-2)} \int d^{2} x \sqrt{g}\left\{-\frac{N+1}{6} R+\left(\frac{f^{\prime 2}}{2 f Z}-\frac{f^{\prime \prime}}{2 Z}\right)\left(\nabla^{\lambda} \chi_{i}\right)\left(\nabla_{\lambda} \chi_{i}\right)\right. \\
& \left.+\left(\frac{N f^{\prime \prime}}{2 f}-\frac{N f^{\prime 2}}{4 f^{2}}-\frac{Z^{\prime 2}}{4 Z^{2}}\right)\left(\nabla^{\lambda} \phi\right)\left(\nabla_{\lambda} \phi\right)+\left(\frac{N f^{\prime}}{2 f}-\frac{Z^{\prime}}{2 Z}\right) \Delta \phi\right\}
\end{aligned}
$$

Here $\phi, \chi_{i}$ denote the dilaton and scalar background correspondently. 
Let us give very brief introduction to the definition of conformal anomaly in two dimensions. It appears as result of quantum breaking (due to regularization) of classical conformal invariance. Hence, it is induced only by quantum effective action. From the definition of quantum energy-momentum tensor for the system which is classically conformally invariant one gets the following:

$$
T=\frac{1}{\sqrt{g}} g^{\mu \nu} \frac{\delta \Gamma}{\delta g^{\mu \nu}} .
$$

This defines conformal anomaly (expectation value of trace of stress-energy tensor). Making the conformal transformation

$$
g_{\mu \nu} \rightarrow \Omega^{2} g_{\mu \nu} \equiv \bar{g}_{\mu \nu}
$$

one can rewrite the conformal anomaly as

$$
T=\left.\frac{\Omega}{\sqrt{g}} \frac{\delta \Gamma}{\delta \Omega}\right|_{\Omega=1} .
$$

Working in the one-loop approximation and reminding that classical action does not give the contribution to conformal anomaly (92) one understands that whole contribution to Eq.(92) is due to Tr log terms. From Eq.(15) one knows that

$$
\bar{\Gamma}^{(1)}=\frac{i}{2} \operatorname{Tr} \ln \left\{\mu^{2} S_{2}\right\}
$$

where dimensional parameter $\mu^{2}$ is explicitly introduced to make $S_{2}$ dimensionless. Under the conformal transformation the one-loop effective action becomes (the case of conformal scalar is considered for simplicity):

$$
\bar{\Gamma}^{(1)}\left[\bar{g}_{\mu \nu}\right]=\frac{i}{2} \operatorname{Tr} \log \left\{\mu^{2} \Omega^{2} S_{2}\right\}
$$

Then

$$
T=\left.\frac{\Omega}{\sqrt{g}} \frac{\delta \bar{\Gamma}^{(1)}}{\delta \Omega}\right|_{\Omega=1}=\left.\frac{\mu(x)}{\sqrt{g}} \frac{\delta \bar{\Gamma}^{(1)}}{\delta \mu(x)}\right|_{\mu=1}
$$

Let us take into account that $\mu$-dependent part of $\bar{\Gamma}^{(1)}$ may be presented as follows (see 48])

$$
\bar{\Gamma}^{(1)}=\int d^{2} x \sqrt{g} a_{1}(x) \frac{1}{2} \ln \mu^{2}+\cdots
$$

where as $\cdots$, we denote the dimensional parameter $\mu$-independent terms. Then Eq.(96) is actually zetaregularization representation of the one-loop effective action. Hence,

$$
T=a_{1}(x)
$$

This $a_{1}(x)$-coefficient of Schwinger-De Witt expansion appears also in the calculation of one-loop divergent part of effective action in dimensional regularization. Hence, multiplying $\Gamma_{d i v}$ to $n-2$ and dropping integral one gets two-dimensional conformal anomaly. Similarly, in four dimensions $T$ is defined by $a_{2^{-}}$ coefficient of Schwinger-De Witt expansion. Note that in such representation of conformal anomaly there may be some ambiguity due to the fact that Schwinger-De Witt coefficients $a_{1}, a_{2}, \cdots$ are defined up to the total derivatives terms. (To solve this problem one should not use the integration by parts in the derivation of $\Gamma_{d i v}$ in Schwinger-De Witt algorithm. This is the case in our calculation!). The good 
algorithm to define the conformal anomaly in universal way is given in ref. 49, where also the problems with total derivative terms in conformal anomaly do not appear.

Thus, one can easily get the conformal anomaly of conformally invariant system just by calculating the corresponding divergent EA multiplied to $(n-2)$ in 2 dimensions (in other words, as coefficient of the pole). Hence, the conformal anomaly of the system under discussion is given by 46 .

$$
\begin{aligned}
T= & \frac{1}{24 \pi}\left\{(N+1) R-3\left(\frac{f^{\prime 2}}{f Z}-\frac{f^{\prime \prime}}{Z}\right)\left(\nabla^{\lambda} \chi_{i}\right)\left(\nabla_{\lambda} \chi_{i}\right)\right. \\
& \left.-3\left(\frac{N f^{\prime \prime}}{f}-\frac{N f^{\prime 2}}{2 f^{2}}-\frac{Z^{\prime 2}}{2 Z^{2}}\right)\left(\nabla^{\lambda} \phi\right)\left(\nabla_{\lambda} \phi\right)-3\left(\frac{N f^{\prime}}{f}-\frac{Z^{\prime}}{Z}\right) \Delta \phi\right\}
\end{aligned}
$$

For purely scalar system coupled with dilaton (i.e. dilaton is classical) the conformal anomaly is simplified:

$$
T=\frac{1}{24 \pi}\left\{N R-3 N\left(\frac{f^{\prime \prime}}{f}-\frac{f^{\prime 2}}{2 f^{2}}\right)\left(\nabla^{\lambda} \phi\right)\left(\nabla_{\lambda} \phi\right)-3 \frac{N f^{\prime}}{f} \Delta \phi\right\}
$$

Anomaly looks very simple in the special case $N=1$ (single scalar). The calculation of such conformal anomaly has been done in the last case, using zeta-regularization method in ref. [50]. Note that the regularization dependence of total derivative terms in conformal anomaly is possible. Thus we got the conformal anomaly for most general $2 \mathrm{~d}$ dilaton coupled scalar-dilaton system with arbitrary dilaton couplings. Similar calculations have been also made in refs. [51, 52, 53, 54, 55, 56] for dilaton coupled scalars. Using the conformal anomaly (see eq.(98)), we may calculate the induced non-local EA.

Let us write the general form of conformal anomaly as following

$$
T=c R+F_{1}(\phi)\left(\nabla^{\lambda} \chi_{i}\right)\left(\nabla_{\lambda} \chi_{i}\right)+F_{2}(\phi)\left(\nabla^{\lambda} \phi\right)\left(\nabla_{\lambda} \phi\right)+F_{3}(\phi) \Delta \phi
$$

where the explicit form of $c, F_{1}, F_{2}, F_{3}$ is evident from the comparison with (99):

$$
\begin{aligned}
& c=\frac{N+1}{24 \pi}, \quad F_{1}(\phi)=-\frac{1}{8 \pi}\left(\frac{f^{\prime 2}}{f Z}-\frac{f^{\prime \prime}}{Z}\right), \\
& F_{2}(\phi)=-\frac{1}{8 \pi}\left(\frac{N f^{\prime \prime}}{f}-\frac{N f^{\prime 2}}{2 f^{2}}-\frac{Z^{\prime 2}}{2 Z^{2}}\right), \quad F_{3}(\phi)=-\frac{1}{8 \pi}\left(\frac{N f^{\prime}}{f}-\frac{Z^{\prime}}{Z}\right) .
\end{aligned}
$$

In situation when dilaton is purely classical the above functions are simplified. The (non-local) effective action induced by the conformal anomaly is defined as

$$
\int d^{2} x \sqrt{g} T=\left.2 \frac{d W}{d t}\right|_{t=1}
$$

where $\tilde{g}^{\mu \nu}=t^{-1} g_{\mu \nu}$. In other words, the effective action (102) should derive the anomaly $T$. Integrating anomaly one gets

$$
\begin{aligned}
W= & -\frac{1}{2} \int d^{2} x \sqrt{g}\left[\frac{c}{2} R \frac{1}{\Delta} R+F_{1}(\phi)\left(\nabla^{\lambda} \chi_{i}\right)\left(\nabla_{\lambda} \chi_{i}\right) \frac{1}{\Delta} R\right. \\
& \left.+\left(F_{2}(\phi)-\frac{\partial F_{3}(\phi)}{\partial \phi}\right) \nabla^{\lambda} \phi \nabla_{\lambda} \phi \frac{1}{\Delta} R+R \int F_{3}(\phi) d \phi\right]
\end{aligned}
$$

The remarkable property of this action is that it is defined for arbitrary dilaton-gravitational background. Note, however, that such action is not yet full effective action. The reason is that it is calculated from 
Eq.(102) as integral from conformal anomaly. Hence, it is defined up to $t$-dependent (conformal factor dependent) constant, or in other words, up to conformally invariant functional.

There is no scheme where one can find such conformally invariant functional in closed form. At best, one can use some kind of approximation to calculate it. Hence, exact and complete expression for one-loop EA looks as

$$
\Gamma=W+\Gamma\left[1, \tilde{g}_{\mu \nu}\right]
$$

where second term is conformally invariant functional. We mention briefly how one can get it. Using SD expansion, in the case of classical dilaton and only quantized scalars, one gets 57

$$
\Gamma\left[1, \tilde{g}_{\mu \nu}\right]=-\frac{N}{4 \pi} \int d^{2} x \sqrt{g}\left(\nabla_{\mu} \phi\right)\left(\nabla^{\mu} \phi\right) \ln \mu^{2}
$$

where numerical factor in front of integral does not matter, as it may be reabsorbed to definition of $\mu^{2}$. Other, non-local terms of this expansion have been recently calculated in ref. [58].

\subsection{Dilaton coupled spinor conformal anomaly in two dimensions}

In the present section, we give another (simpler) method [59] to find conformal anomaly for dilaton coupled spinor. Unfortunately, it cannot be applied to other theories.

Let us start from $2 \mathrm{~d}$ dilaton coupled spinor Lagrangian:

$$
L=\sqrt{-g} f(\phi) \bar{\psi} \gamma^{\mu} \partial_{\mu} \psi
$$

where $\psi$ is $2 \mathrm{~d}$ Majorana spinor.]

Let us make now the following classical transformation of background field $g_{\mu \nu}: g_{\mu \nu} \rightarrow f^{-2}(\phi) \tilde{g}_{\mu \nu}$. Then it is easy to see that $\gamma^{\mu}(x) \rightarrow f(\phi) \tilde{\gamma}^{\mu}(x)$ and in terms of new classical metric we obtain usual, non-coupled with dilaton (minimal) Lagrangian for $2 \mathrm{~d}$ spinor:

$$
L=\sqrt{-\tilde{g}} \bar{\psi} \tilde{\gamma}^{\mu} \partial_{\mu} \psi
$$

The conformal anomaly for Lagrangian (107) is well-known:

$$
\sqrt{-\tilde{g}} T=\frac{\sqrt{-\tilde{g}}}{24 \pi}\left\{\frac{1}{2} \tilde{R}\right\} .
$$

Now, transforming Eq. (108) to original variables: $\tilde{g}_{\mu \nu}=f^{2}(\phi) g_{\mu \nu}, \tilde{R}=f^{-2}(\phi)(R-2 \Delta \ln f)$, we get the following conformal anomaly for dilaton coupled Majorana spinor (106):

$$
\sqrt{-g} T=\frac{\sqrt{-g}}{24 \pi}\left[\frac{1}{2} R-\Delta \ln f\right]=\frac{\sqrt{-g}}{24 \pi}\left[\frac{1}{2} R-\frac{f^{\prime}}{f} \Delta f-\frac{\left(f^{\prime \prime} f-f^{\prime 2}\right)}{f^{2}} g^{\mu \nu} \partial_{\mu} \phi \partial_{\nu} \phi\right] .
$$

\footnotetext{
${ }^{3}$ As we see below in the present formalism where only notations for classical external fields are changed the dilatonic contribution to conformal anomaly appears. This contribution has the form of total derivative term. In ref. 56 using transformations of quantum fields,Fujikawa regularization and the proper choice of integration measure the calculation of dilaton dependent contribution to conformal anomaly gives zero. This fact maybe regarded as the regularization dependence of conformal anomaly. It is not strange as it is known for some time that total derivative terms of conformal anomaly in various dimensions may depend on the choice of regularization. Moreover, in dilaton dependent SUSY theories like super YM coupled with conformal supergravity (where dilaton presents) the fact of the presence of dilaton dependent terms in conformal anomaly is proven by various formalisms. It indicates that from SUSY point of view the measure of integration in functional space which leads to non-trivial dilaton dependent term in conformal anomaly looks more physical.
} 
For 2d dilaton coupled Dirac spinor the conformal anomaly is twice of that for Majorana spinor. Note also that it could be as in case of dilaton coupled scalar, conformal anomaly (its total derivative terms) depends on the regularization.

In the conformal anomaly (109) dilaton dependent terms appear in the form of total derivative. In principle, it means that this term is ambiguous by physical reasons. Indeed, in two dimensions the analogue of Einstein action looks like:

$$
S=\frac{1}{G} \int d^{2} x \sqrt{-g} R f(\phi)
$$

Now, there exists the following relation

$$
g_{\mu \nu} \frac{\delta}{\delta g_{\mu \nu}} \int d^{2} x \sqrt{-g} R f(\phi)=\Delta f(\phi) .
$$

In other words, by finite renormalization of gravitational action (110), we can always change the coefficient of $\Delta f$ term in conformal anomaly. So in $2 \mathrm{~d}$ gravity this term is only fixed by the physical renormalization condition. That is also why total derivative term of conformal anomaly for dilaton coupled scalar is ambiguous.

Now we discuss the anomaly induced effective action for dilaton coupled spinor. The derivation goes in the same way as it was for dilaton coupled scalar. Making the conformal transformation of the metric $g_{\mu \nu} \rightarrow \mathrm{e}^{2 \sigma} g_{\mu \nu}$ in the conformal anomaly, and using relation

$$
\sqrt{-g} T=\frac{\delta}{\delta \sigma} W[\sigma]
$$

one can find the anomaly induced effective action $W$. In the covariant, non-local form it may be written as following:

$$
W=-\frac{1}{2} \int d^{2} x \sqrt{-g}\left\{\frac{1}{96 \pi} R \frac{1}{\Delta} R+\left(F_{2}(\phi)-\frac{\partial F_{3}(\phi)}{\partial \phi}\right)\left(\nabla^{\lambda} \phi\right)\left(\nabla_{\lambda} \phi\right) \frac{1}{\Delta} R+R \int F_{3}(\phi) d \phi\right\}
$$

where $F_{2}(\phi)=-\frac{f^{\prime \prime} f-f^{\prime 2}}{24 \pi f^{2}}, F_{3}(\phi)=-\frac{f^{\prime}}{24 \pi f}$. Note that coefficient of second term is actually zero as is easy to check. Hence, we got anomaly induced effective action for dilaton coupled spinor.

An interesting thing is that the effective action 113 exactly reproduces the effective action of RST model [99], which is exactly solvable. The RST model is given by adding the quantum correction

$$
W_{R S T}=-\frac{1}{2} \int d^{2} x \sqrt{-g}\left\{\frac{N}{48 \pi} R \frac{1}{\Delta} R+\frac{N}{24 \pi} \phi R\right\}
$$

to the action of the CGHS model 35]

$$
S_{C G H S}=\frac{1}{2 \pi} \int d^{2} x \sqrt{-g} \mathrm{e}^{-2 \phi}\left(R+4 \nabla_{\mu} \phi \nabla^{\mu} \phi+4 \lambda^{2}\right) .
$$

In [99], the second term in $W_{R S T}$ (114) is added by hands.

It is interesting that full EA is again defined with accuracy up to conformally invariant functional. To conclude this section let us note that in calculation of EA for consistent $2 \mathrm{~d}$ dilatonic gravity with matter one needs also quantum gravitational contribution. However, dilatonic gravity is not renormalizable in the standard way as it was shown in previous chapter. As a result finite contributions to effective action will depend on regularization scheme under discussion. 


\subsection{Induced effective action for $4 \mathrm{~d}$ dilaton coupled scalar}

It could be interesting to generalize the results of previous sections for $4 \mathrm{~d}$ case. The purpose of the present section will be to calculate the non-local effective action for $4 \mathrm{~d}$ dilaton coupled conformal scalar 47. Let us consider the theory with the following Lagrangian in curved spacetime

$$
L=\sqrt{-g} \varphi f(\phi)(\square-\xi R) \varphi
$$

where $\varphi$ is quantum scalar field, $\square=g^{\mu \nu} \nabla_{\mu} \nabla_{\nu}$, $\phi$ is an external field (dilaton), $f(\phi)$ is an arbitrary function. It is very easy to check that the theory (116) is conformally invariant for $\xi=\frac{1}{6}$.

Let us calculate the divergent part of the effective action for the theory (116):

$$
\begin{aligned}
\Gamma_{\text {div }} & =-\frac{i}{2} \operatorname{Tr} \ln \left\{f(\phi)\left[\square-\xi R+\frac{\square f(\phi)}{2 f(\phi)}+\frac{\left(\nabla^{\mu} f(\phi)\right)}{f(\phi)} \nabla_{\mu}\right]\right\} \\
& =\frac{1}{(n-4)} \int d^{4} x \sqrt{-g} b_{4}
\end{aligned}
$$

where $b_{4}$ (or $a_{2}$ ) is the $b_{4}$-coefficient of Schwinger-De Witt expansion. Applying standard methods, we find $b_{4}$ explicitly, where $f(\phi)$-multiplier in Eq. 117 does not contribute. For $\xi=\frac{1}{6}$, we get the conformal anomaly which is equal to $b_{4}$

$$
T=\frac{1}{(4 \pi)^{2}}\left\{\frac{1}{32} \frac{[(\nabla f)(\nabla f)]^{2}}{f^{4}}+\frac{1}{24} \square\left(\frac{(\nabla f)(\nabla f)}{f^{2}}\right)+\frac{1}{180}\left(R_{\mu \nu \alpha \beta}^{2}-R_{\mu \nu}^{2}+\square R\right)\right\}
$$

Here, the last term is the well-known Weyl anomaly (for a review, see [1, 60]) for conformally invariant scalar. The first two terms in (118) are the dilaton contribution to conformal anomaly.

Let us write the Eq.(118) in a slightly different form:

$$
T=\left\{b\left(F+\frac{2}{3} \square R\right)+b^{\prime} G+b^{\prime \prime} \square R+a_{1} \frac{[(\nabla f)(\nabla f)]^{2}}{f^{4}}+a_{2} \square\left(\frac{(\nabla f)(\nabla f)}{f^{2}}\right)\right\}
$$

where $F$ is the square of Weyl tensor in four dimensions, $G$ is Gauss-Bonnet invariant. For scalar field, it follows from (118) that $b=\frac{1}{120(4 \pi)^{2}}, b^{\prime}=-\frac{1}{360(4 \pi)^{2}}, a_{1}=\frac{1}{32(4 \pi)^{2}}, a_{2}=\frac{1}{24(4 \pi)^{2}}$ and in principle $b^{\prime \prime}$ is an arbitrary parameter (it may be changed by the finite renormalization of local counterterm).

The non-local effective action induced by the conformal anomaly (without dilaton) has been calculated sometime ago [61]. Using Eq.(112) and integrating it, one can restore the non-local effective action $W$ induced by the conformal anomaly:

$$
\begin{aligned}
W & =b \int d^{4} x \sqrt{-g} F \sigma+b^{\prime} \int d^{4} x \sqrt{-g}\left\{\sigma\left[2 \square^{2}+4 R^{\mu \nu} \nabla_{\mu} \nabla_{\nu}-\frac{4}{3} R \square+\frac{2}{3}\left(\nabla^{\mu} R\right) \nabla_{\mu}\right] \sigma\right. \\
+ & \left.\left(G-\frac{2}{3} \square R\right) \sigma\right\}-\frac{1}{12}\left(b^{\prime \prime}+\frac{2}{3}\left(b+b^{\prime}\right)\right) \int d^{4} x \sqrt{-g}[R-6 \square \sigma-6(\nabla \sigma)(\nabla \sigma)]^{2} \\
+ & \int d^{4} x \sqrt{-g}\left\{a_{1} \frac{[(\nabla f)(\nabla f)]^{2}}{f^{4}} \sigma+a_{2} \square\left(\frac{(\nabla f)(\nabla f)}{f^{2}}\right) \sigma\right. \\
& \left.+a_{2} \frac{(\nabla f)(\nabla f)}{f^{2}}[(\nabla \sigma)(\nabla \sigma)]\right\}
\end{aligned}
$$

Here the $\sigma$-independent term is dropped, last terms represent the contribution from the dilaton dependent terms in conformal anomaly. It is interesting that dilatonic contribution may be important in the study of quantum irreversibility [62]. 


\subsection{Conformal and chiral anomaly for $4 \mathrm{~d}$ dilaton coupled spinor}

We consider now 4d dilaton coupled fermion which may appear as the result of spherical reduction of higher dimensional minimal spinor (we follow to ref.[59]). The corresponding conformally invariant Lagrangian may be taken as follows 1

$$
L=\sqrt{-g} f(\phi) \bar{\psi} \gamma^{\mu}(x) \nabla_{\mu} \psi
$$

where $\nabla_{\mu}=\partial_{\mu}+\frac{1}{2} \omega_{\mu}^{a b} \sigma_{a b}$.

Let us make the following transformation of background gravitational field:

$$
g_{\mu \nu}=\mathrm{e}^{2 \sigma(x)} \tilde{g}_{\mu \nu}, \quad \gamma^{\mu}(x)=\mathrm{e}^{-\sigma(x)} \tilde{\gamma}^{\mu}(x), \quad \sqrt{-g}=\mathrm{e}^{4 \sigma(x)} \sqrt{-\tilde{g}} .
$$

where $\sigma(x)$ satisfies $\mathrm{e}^{3 \sigma(x)} f(\phi)=1$. Then, Lagrangian (121) after transformation (122) takes the form:

$$
L=\sqrt{-\tilde{g}}\left[\bar{\psi} \tilde{\gamma}^{\mu}(x) \tilde{\nabla}_{\mu} \psi+\bar{\psi} \tilde{\gamma}^{\mu} \tilde{A}_{\mu} \psi\right]
$$

where we used $\partial_{\mu} \equiv \tilde{\partial}_{\mu}$ and $\tilde{A}_{\mu}=\frac{3}{2} \partial_{\mu} \sigma(x)$. Note that field strength for above vector field is equal to zero, that is why no terms of the sort : square of field strength for above vector appear in conformal anomaly. Hence, the calculation of $a_{2}$ Schwinger-De Witt coefficient in theory (121) in curved spacetime with nontrivial dilaton is equivalent to the calculation of $a_{2}$ in an external gravitational field $\tilde{g}_{\mu \nu}$ (but no dilaton) and external vector field $\tilde{A}_{\mu}$. Conformal anomaly for such quantum (Dirac) fermion is wellknown:

$$
\frac{\sqrt{-\tilde{g}}}{(4 \pi)^{2}}\left\{\frac{1}{20}\left(\tilde{F}+\frac{2}{3} \square \tilde{R}\right)-\frac{11}{360} \tilde{G}\right\}
$$

where $\tilde{F}=\tilde{R}_{\mu \nu \alpha \beta} \tilde{R}^{\mu \nu \alpha \beta}-2 \tilde{R}_{\mu \nu} \tilde{R}^{\mu \nu}+\frac{1}{3} \tilde{R}^{2}, \tilde{G}=\tilde{R}_{\mu \nu \alpha \beta} \tilde{R}^{\mu \nu \alpha \beta}-4 \tilde{R}_{\mu \nu} \tilde{R}^{\mu \nu}+\tilde{R}^{2}$. One can also present coefficients of conformal anomaly as $b=\frac{1}{20(4 \pi)^{2}}$ and $b^{\prime}=-\frac{11}{360(4 \pi)^{2}}$. Now, one can transform the relation (124) back to original metric tensor. It is easy to see that there are dilaton dependent contributions to conformal anomaly. One can also find anomaly induced effective action. Starting from Eq.(124) for conformal anomaly in terms of tilded metric the corresponding anomaly induced action in this case is quite known (see 61, 63, 64, 43, 44):

$$
\begin{aligned}
W= & -\frac{1}{4 b^{\prime}} \int d^{4} x \sqrt{-\tilde{g}} \int d^{4} x^{\prime} \sqrt{-\tilde{g}^{\prime}}\left[b \tilde{F}+b^{\prime}\left(\tilde{G}-\frac{2}{3} \square \tilde{R}\right)\right]_{x} \\
& \times\left[2 \square^{2}+4 \tilde{R}^{\mu \nu} \tilde{\nabla}_{\mu} \tilde{\nabla}_{\nu}-\frac{4}{3} \tilde{R} \square+\frac{2}{3}\left(\tilde{\nabla}^{\mu} \tilde{R}\right) \tilde{\nabla}_{\mu}\right]_{x x^{\prime}}^{-1} \\
& \times\left[b \tilde{F}+b^{\prime}\left(\tilde{G}-\frac{2}{3} \square \tilde{R}\right)\right]_{x^{\prime}}-\frac{1}{18}\left(b+b^{\prime}\right) \int d^{4} x \sqrt{-\tilde{g}} \tilde{R}^{2} .
\end{aligned}
$$

It is trivial to substitute metric $\tilde{g}_{\mu \nu}=\mathrm{e}^{-2 \sigma} g_{\mu \nu}, \sigma=-\frac{1}{3} \ln f(\phi)$ and rewrite above equation in terms of original metric and dilaton function but the final result is a bit complicated.

\footnotetext{
${ }^{4}$ As we mentioned in section 3.2 in the present formalizm where no transformations of quantum fields is done we get the dilaton dependent contribution to conformal anomaly. Such dilaton dependent contribution to conformal anomaly looks very natural from SUSY point of view. In ref. 56 in terms of Fujikawa regularization the other answer has been obtained. The difference in two answers maybe regarded as regularization dependence of conformal anomaly.
} 
As final remark, we note that similarly one can calculate the chiral anomaly for dilaton coupled spinor. Chiral anomaly for theory (123) is known [65, 66, 67]:

$$
\begin{aligned}
A_{\frac{1}{2}}= & \frac{2 i}{(4 \pi)^{2}}\left[-\frac{1}{48} \epsilon^{\mu \nu \rho \sigma} \tilde{R}_{\zeta \mu \nu}^{\xi} \tilde{R}_{\xi \rho \sigma}^{\zeta}\right] \\
= & \frac{2 i}{(4 \pi)^{2}}\left[-\frac{1}{48} \epsilon^{\mu \nu \rho \sigma}\left\{R_{\zeta \mu \nu}^{\xi} R_{\xi \rho \sigma}^{\zeta}-16 R_{\rho \mu \nu}^{\xi} \nabla_{\sigma} \nabla_{\xi} \sigma\right.\right. \\
& \left.\left.-16 R_{\rho \mu \nu}^{\xi} \nabla_{\sigma} \sigma \nabla_{\xi} \sigma-8 R_{\mu \nu \rho \sigma} \nabla_{\alpha} \nabla^{\alpha} \sigma\right\}\right] .
\end{aligned}
$$

Hence we found explicitly dilaton dependent corrections for chiral and conformal anomaly in the theory of $4 \mathrm{~d}$ dilaton coupled spinor.

\subsection{Conformal anomaly for $4 \mathrm{~d}$ dilaton coupled vector field}

In the study of string theory the low energy $4 \mathrm{~d}$ string effective action may be presented as following

$$
S=\int d^{4} x \sqrt{-g}\left[R+4(\nabla \phi)^{2}+F_{\mu \nu}^{2}\right] \mathrm{e}^{-2 \phi}
$$

where $\phi$ is dilaton, $F_{\mu \nu}=\nabla_{\mu} A_{\nu}-\nabla_{\nu} A_{\mu}, A_{\mu}$ is electromagnetic field. Investigating of string gravity (127) the quantum effects of electromagnetic field with the lagrangian:

$$
L=-\frac{1}{4} f(\phi) F_{\mu \nu} F^{\mu \nu}
$$

may be dominant in some regions,especially if we consider generalization of above model with $N$ vectors and apply large $N$ expansion. From another point if we start from usual Einstein-Maxwell gravity in $d$-dimensions, we can do spherical reduction to the space $R_{4} \times S_{d-4}$ where $R_{4}$ is an arbitrary curved space. Then the reduced action becomes again of the form (127) (maybe with change of some terms and some coefficients) where the radius of $S_{d-4}$ plays the role of dilatonic function.

Hence, the study of dilaton coupled electromagnetic field with the Lagrangian (127) which describes conformally invariant theory may be of interest in different respects. In the present section, we study conformal anomaly in the theory (127) in four dimensions (in two dimensions theory is not conformally invariant).

Adding to the Lagrangian (128) the gauge-fixing Lagrangian $L_{g f}$ :

$$
L_{g f}=-\frac{1}{2} f(\phi)\left(\nabla_{\nu} A^{\nu}\right)^{2}
$$

one can apply the standard algorithm to get the effective action. For ghosts it is the same as in the theory with no dilaton

$$
\begin{aligned}
& \Gamma_{\text {ghost }}^{(1)}=\frac{1}{(4 \pi)^{2}(n-4)} \int d^{4} x \sqrt{-g}\left\{-\frac{1}{90} R_{\mu \nu \alpha \beta}^{2}+\frac{1}{90} R_{\mu \nu}^{2}-\frac{R^{2}}{36}-\frac{1}{15} \square R\right\} \\
& \quad=\frac{1}{(n-4)} \int d^{4} x \sqrt{-g} b_{4}^{\text {ghost }} .
\end{aligned}
$$

The one-loop effective action due to vectors may be found as 68, 56

$$
\Gamma_{A_{\mu}}^{(1)}=\frac{1}{(4 \pi)^{2}(n-4)} \int d^{4} x \sqrt{-g}\left\{-\frac{11}{180} R_{\mu \nu \alpha \beta}^{2}+\frac{43}{90} R_{\mu \nu}^{2}-\frac{1}{9} R^{2}\right.
$$




$$
\begin{aligned}
& -\frac{1}{30} \square R-\frac{1}{3} \square\left(\frac{1}{f} \square f\right)+\frac{5}{12} \square\left[\frac{1}{f^{2}}\left(\nabla_{\alpha} f\right)\left(\nabla^{\alpha} f\right)\right] \\
& -\frac{1}{3 f^{2}} R_{\mu \nu}\left(\nabla^{\mu} f\right)\left(\nabla^{\nu} f\right)-\frac{1}{3 f} R_{\mu \nu}\left(\nabla^{\mu} \nabla^{\nu} f\right)+\frac{R}{6 f}(\square f) \\
& +\frac{9}{16 f^{4}}\left(\nabla_{\mu} f\right)\left(\nabla^{\mu} f\right)\left(\nabla_{\alpha} f\right)\left(\nabla^{\alpha} f\right)+\frac{1}{6 f^{3}}\left(\nabla^{\beta} f\right)\left(\nabla^{\nu} f\right)\left(\nabla_{\beta} \nabla_{\nu} f\right) \\
& \left.-\frac{11}{12 f^{3}}\left(\nabla^{\mu} f\right)\left(\nabla_{\mu} f\right)(\square f)-\frac{1}{6 f^{2}}\left(\nabla_{\alpha} \nabla_{\beta} f\right)\left(\nabla^{\alpha} \nabla^{\beta} f\right)+\frac{5}{12 f^{2}}(\square f)(\square f)\right\} \\
& =\frac{1}{(n-4)} \int d^{4} x \sqrt{-g} b_{4}^{\text {vector }} .
\end{aligned}
$$

The total one-loop effective action $\Gamma^{(1)}$ is given by sum of (130) and (131). From the expression for $\Gamma^{(1)}$ one can easily get conformal anomaly: $T=b_{4}=b_{4}^{\text {ghost }}+b_{4}^{\text {vector }}$. The first four terms give the well-known conformal anomaly of electromagnetic field [69, 70]. We have to note that using another regularization (like zeta-regularization) may slightly alter the coefficients of total derivative terms, like already happened in this case in the absence of dilaton [69, 70].

We have to note, however, that there are some unclear points. First, it was shown 68, 56 that such conformal anomaly cannot be integrated and anomaly induced action cannot be constructed. That indicates that convenient approach (as applied above) fails to reproduce correct conformal anomaly. It is known 60 that conformal anomaly consists from only trivial (total derivative) terms and conformally invariant terms. Above conformal anomaly does not satisfy this property as it was shown in [56] via classification of terms entering to conformal anomaly. It may be that BRST approach (for a recent review, see 71]) in combination with some convenient regularization may help in resolution of this problem as it was discussed in [56]. In the last chapter, we will show that correct conformal anomaly for this case may be reproduced from AdS/CFT correspondence. Nevertheless, it would be interesting to understand why conventional approach to conformal anomaly as in this section fails.

\subsection{Conformal anomaly and induced action in $2 \mathrm{~d}$ dilatonic supergravity with dilaton coupled Wess-Zumino matter}

Based on [72], in this section, we construct the theory of $2 d$ dilatonic supergravity(SG) with matter and dilaton supermultiplets coupled to dilaton functions. The $2 \mathrm{~d}$ model also appears by the spherical reduction from $4 \mathrm{~d}$ supergravity theories $[72$. Conformal anomaly and induced effective action for matter supermultiplet are calculated, which gives also large- $N$ effective action for dilatonic SG.

At first we construct the action of 2 d dilatonic supergravity with dilaton supermultiplet and with matter supermultiplet. In order to find the Lagrangian of $2 \mathrm{~d}$ dilatonic supergravity, we use the component formulation of ref. 73, 74, 75. (for introduction, see book 76). Here, all the scalar fields are real and all the spinor fields are Majorana spinors. We introduce dilaton multiplet $\Phi=(\phi, \chi, F)$ and matter multiplet $\Sigma_{i}=\left(a_{i}, \chi_{i}, G_{i}\right)$, which has the conformal weight $\lambda=0$, and the curvature multiplet $W$

$$
W=\left(S, \eta,-S^{2}-\frac{1}{2} R-\frac{1}{2} \bar{\psi}^{\mu} \gamma^{\nu} \psi_{\mu \nu}+\frac{1}{4} \bar{\psi}^{\mu} \psi_{\mu}\right)
$$


Here $R$ is the scalar curvature and

$$
\begin{aligned}
& \eta=-\frac{1}{2} S \gamma^{\mu} \psi_{\mu}+\frac{i}{2} e^{-1} \epsilon^{\mu \nu} \gamma_{5} \psi_{\mu \nu}, \quad \psi_{\mu \nu}=D_{\mu} \psi_{\nu}-D_{\nu} \psi_{\mu} \\
& D_{\mu} \psi_{\nu}=\left(\partial_{\mu}-\frac{1}{2} \omega_{\mu} \gamma_{5}\right) \psi_{\nu}, \quad \omega_{\mu}=-i e^{-1} e_{a \mu} \epsilon^{\lambda \nu} \partial_{\lambda} e_{\nu}^{a}-\frac{1}{2} \bar{\psi}_{\mu} \gamma_{5} \gamma^{\lambda} \psi_{\lambda}
\end{aligned}
$$

The curvature multiplet has the conformal weight $\lambda=1$.

Then the general action of $2 \mathrm{~d}$ dilatonic supergravity is given in terms of general functions of the dilaton $C(\phi), Z(\phi), f(\phi)$ and $V(\phi)$ as follows

$$
\begin{aligned}
& \mathcal{L}=-[C(\Phi) \otimes W]_{\mathrm{inv}}+[V(\Phi)]_{\mathrm{inv}}+\frac{1}{2}\left[\Phi \otimes \Phi \otimes T_{P}(Z(\Phi))\right]_{\mathrm{inv}}-\left[Z(\Phi) \otimes \Phi \otimes T_{P}(\Phi)\right]_{\mathrm{inv}} \\
& +\sum_{i=1}^{N}\left\{\frac{1}{2}\left[\Sigma_{i} \otimes \Sigma_{i} \otimes T_{P}(f(\Phi))\right]_{\mathrm{inv}}-\left[f(\Phi) \otimes \Sigma_{i} \otimes T_{P}\left(\Sigma_{i}\right)\right]_{\mathrm{inv}}\right\} .
\end{aligned}
$$

$T_{P}(Z)$ is called the kinetic multiplet for the multiplet $Z=(\varphi, \zeta, H)$ and when the multiplet $Z$ has the comformal weight $\lambda=0, T_{P}(Z)$ has the following form

$$
T_{P}(Z)=(H, D \zeta, \square \varphi)
$$

The kinetic multiplet $T_{P}(Z)$ has conformal weight $\lambda=1$. The product of two multiplets $Z_{k}=\left(\varphi_{k}, \zeta_{k}, H_{k}\right)$ $(k=1,2)$ with the conformal weight $\lambda_{k}$ is defined by

$$
Z_{1} \otimes Z_{2}=\left(\varphi_{1} \varphi_{2}, \varphi_{1} \zeta_{2}+\varphi_{2} \zeta_{1}, \varphi_{1} H_{2}+\varphi_{2} H_{1}-\bar{\zeta}_{1} \zeta_{2}\right)
$$

The invariant Lagrangian $[Z]_{\text {inv }}$ for multiplet $Z$ is defined by

$$
[Z]_{\mathrm{inv}}=e\left[F+\frac{1}{2} \bar{\psi}_{\mu} \gamma^{\mu} \zeta+\frac{1}{2} \varphi \bar{\psi}_{\mu} \sigma^{\mu \nu} \psi_{\nu}+S \varphi\right] .
$$

Then if necessary one can rewrite the Lagrangian (134) in lengthy component form. The covariant derivatives for the multiplet $Z=(\varphi, \zeta, H)$ with $\lambda=0$ are defined as

$$
\begin{aligned}
& D_{\mu} \varphi=\partial_{\mu} \varphi-\frac{1}{2} \bar{\psi}_{\mu} \zeta, \quad D_{\mu} \zeta=\left(\partial_{\mu}+\frac{1}{2} \omega_{\mu} \gamma_{5}\right) \zeta-\frac{1}{2} D_{\nu} \varphi \gamma^{\nu} \psi_{\mu}-\frac{1}{2} H \psi_{\mu}, \\
& \square \varphi=e^{-1}\left\{\partial_{\nu}\left(e g^{\mu \nu} D_{\mu} \varphi\right)+\frac{i}{4} \bar{\zeta} \gamma_{5} \psi_{\mu \nu} \epsilon^{\mu \nu}-\frac{1}{2} \bar{\psi}^{\mu} D_{\mu} \zeta-\frac{1}{2} \bar{\psi}^{\mu} \gamma^{\nu} \psi_{\nu} D_{\mu} \varphi\right\} .
\end{aligned}
$$

The action (134) is, by construction, invariant under the following local supersymmetry transformation,

$$
\begin{aligned}
& \delta e_{\mu}^{a}=\bar{\epsilon} \gamma^{a} \psi_{\mu}, \delta \psi_{\mu}=2\left(\partial_{\mu}+\frac{1}{2} \omega_{\mu} \gamma_{5}+\frac{1}{2} \gamma_{\mu} S\right) \epsilon, \delta S=-\frac{1}{2} S \bar{\epsilon} \gamma^{\mu} \psi_{\mu}+\frac{1}{2} i e^{-1} \epsilon^{\mu \nu} \bar{\epsilon} \gamma_{5} \psi_{\mu \nu} \\
& \delta \phi=\bar{\epsilon} \chi, \quad \delta \chi=\left\{F+\gamma^{\mu}\left(\partial_{\mu} \phi-\frac{1}{2} \bar{\psi}_{\mu} \chi\right)\right\} \epsilon \\
& \delta F=\bar{\epsilon} \gamma^{\mu}\left\{\left(\partial_{\mu}+\frac{1}{2} \omega_{\mu} \gamma_{5}\right) \chi-\frac{1}{2} \gamma^{\nu}\left(\partial_{\nu} A-\frac{1}{2} \bar{\psi}_{\nu} \chi\right) \psi_{\mu}-\frac{1}{2} F \psi_{\mu}\right\} \\
& \delta a_{i}=\bar{\epsilon} \xi_{i}, \quad \delta \xi_{i}=\left\{G_{i}+\gamma^{\mu}\left(\partial_{\mu} a_{i}-\frac{1}{2} \bar{\psi}_{\mu} \xi_{i}\right)\right\} \epsilon \\
& \delta G_{i}=\bar{\epsilon} \gamma^{\mu}\left\{\left(\partial_{\mu}+\frac{1}{2} \omega_{\mu} \gamma_{5}\right) \xi_{i}-\frac{1}{2} \gamma^{\nu}\left(\partial_{\nu} \phi-\frac{1}{2} \bar{\psi}_{\nu} \xi_{i}\right) \psi_{\mu}-\frac{1}{2} G_{i} \psi_{\mu}\right\} .
\end{aligned}
$$

Here $\epsilon$ is an anti-commuting spinor parameter of local supersymmetry transformation and $\omega_{\mu}$ is the spin connection and given by $\omega_{\mu}=-i e^{-1} e_{a \mu} \epsilon^{\lambda \nu} \partial_{\lambda} e_{\nu}^{a}-\frac{1}{2} \bar{\psi}_{\mu} \gamma_{5} \gamma^{\lambda} \psi_{\lambda}$. Hence, we constructed the classical 
action for $2 \mathrm{~d}$ dilatonic supergravity with dilaton and matter supermultiplets. The extended versions of $2 \mathrm{~d}$ dilatonic supergravity have been constructed in refs. [77, 78.

We now study the conformal anomaly and effective action in large- $N$ approximation for the $2 \mathrm{~d}$ dilatonic supergravity. We consider only bosonic background below as it will be sufficient for the study of black hole or cosmological applications. Then, the dilatino $\chi$ and the Rarita-Schwinger fields vanish, and one can show that gravity and dilaton part of the Lagrangian has the following form:

$$
\begin{aligned}
& {[C(\Phi) \otimes W]_{\mathrm{inv}}=e\left[-C(\phi)\left(S^{2}+\frac{1}{2} R\right)-C^{\prime}(\phi) F S\right],} \\
& {\left[\Phi \otimes \Phi \otimes T_{P}(Z(\Phi))\right]_{\mathrm{inv}}=e\left[\phi^{2} \square(Z(\phi))+2 Z^{\prime}(\phi) \phi F^{2}\right],} \\
& {\left[Z(\Phi) \otimes \Phi \otimes T_{P}(\Phi)\right]_{\mathrm{inv}}=e\left[Z(\phi) \phi \square \phi+Z^{\prime}(\phi) \phi F^{2}+Z(\phi) F^{2}\right],} \\
& {[V(\Phi)]_{\mathrm{inv}}=e\left[V^{\prime}(\phi) F+S V(\phi)\right] .}
\end{aligned}
$$

Using the equations of motion with respect to the auxiliary fields $S, F, G_{i}$, on the bosonic background one can show that

$$
S=\frac{C^{\prime}(\phi) V^{\prime}(\phi)-2 V(\phi) Z(\phi)}{C^{\prime 2}(\phi)+4 C(\phi) Z(\phi)}, F=\frac{C^{\prime}(\phi) V(\phi)+2 C(\phi) V^{\prime}(\phi)}{C^{\prime 2}(\phi)+4 C(\phi) Z(\phi)}, G_{i}=0 .
$$

For the matter on bosonic background

$$
\begin{aligned}
& \sum_{i=1}^{N}\left\{\frac{1}{2}\left[\Sigma_{i} \otimes \Sigma_{i} \otimes T_{P}(f(\Phi))\right]_{\mathrm{inv}}-\left[f(\Phi) \otimes \Sigma_{i} \otimes T_{P}\left(\Sigma_{i}\right)\right]_{\mathrm{inv}}\right\} \\
& =e f(\phi) \sum_{i=1}^{N}\left(g^{\mu \nu} \partial_{\mu} a_{i} \partial_{\nu} a_{i}+\bar{\xi}_{i} \gamma^{\mu} \partial_{\mu} \xi_{i}-f(\phi) G_{i}^{2}\right)+\left(\begin{array}{c}
\text { total divergence } \\
\text { terms }
\end{array}\right)
\end{aligned}
$$

Here we have used the fact that $\bar{\xi}_{i} \gamma_{5} \xi=0$ for the Majorana spinor $\xi_{i}$.

Let us write down the effective action in above theory. It is clearly seen that theory (142) is conformally invariant on the gravitational background under discussion. Then using standard methods, we can prove that theory with matter multiplet $\Sigma_{i}$ is superconformally invariant theory. The anomaly induced effective action due to $N$ scalars and spinors may be derived from Eqs.(103) and (113) as the following:

$$
W=-\frac{1}{2} \int d^{2} x \sqrt{-g}\left\{\frac{N}{32 \pi} R \frac{1}{\Delta} R-\frac{N}{16 \pi} \frac{f^{\prime 2}}{f^{2}}\left(\nabla^{\lambda} \phi\right)\left(\nabla_{\lambda} \phi\right) \frac{1}{\Delta} R-\frac{N}{6 \pi} R \ln f\right\} .
$$

Thus, using results of first sections of this chapter we were able to get the effective action for dilatonic supergravity with matter. It is interesting to note that similarly one can get large $\mathrm{N}$, anomaly induced effective action for dilatonic supergravity (with matter) in other dimensions. In the next chapter we discuss applications of induced effective action in black holes thermodynamics with account of quantum corrections and in chapter 5- in quantum cosmology.

\section{Thermodynamics of quantum dilatonic black holes}

Our purpose in this chapter will be to apply induced effective action in the study of quantum aspects of black holes thermodynamics. Let us first make few general remarks. 
When we try to construct the quantum gravity theory, black hole evaporation provides a serious problem. A quantum mechanically pure state which describes gravitationally collapsing matter to form a black hole evolves into a mixed quantum state, which describes Hawking radiation [79]. There are several scenarios to solve this problem of the loss of quantum information. Hawking gave a most radical proposal that the black hole completely evaporates and the quantum coherence is lost in the gravitational collapse. On the other hand, 't Hooft has proposed that Hawking radiation carries off information about the quantum states of the black holes [80]. Another conservative proposal is that the process of the collapse and the radiation leaves a stable remnant which carries the information of the initial configuration of the system [81]. However, if the remnant has the mass of order the Planck scale, gravitational effects would produce the light remnants in pairs and the lifetime of stars would be shorter than observed. Therefore the mass of the remnants should be macroscopic. A candidate of such remnants is the extremal black hole of Reissner-Nordstrøm type 82. The extremal black holes have vanishing temperature and the space-time metric is not singular everywhere.

\subsection{2d dilatonic black holes and Hawking radiation}

Let us turn now to overview of the $2 \mathrm{~d}$ dilatonic black holes properties. Callan, Giddings, Harvey and Strominger [35] have investigated an interesting toy model of two dimensional gravity, which contains Hawking radiation and which is now called CGHS model. The action of the model is given by adding the action of $N$ minimal scalar fields $a_{i}(i=1, \cdots, N)$

$$
S_{a}=-\frac{1}{2 \pi} \int d^{2} x \sqrt{-g} \sum_{j=1}^{N} \partial_{\mu} a_{j} \partial^{\mu} a_{j}
$$

to the action (115). When we fix the gauge freedom of reparametrization invariance by the conformal gauge,

$$
g_{\mp \pm}=-\frac{1}{2} \mathrm{e}^{2 \rho}, \quad g_{ \pm \pm}=0,
$$

the general solutions of eqs. of motion are given by,

$$
\begin{aligned}
& a_{j}=a_{j}^{+}\left(x^{+}\right)+a_{j}^{-}\left(x^{-}\right), \\
& \mathrm{e}^{-2 \phi}=\mathrm{e}^{-2 \rho}=\frac{M}{\lambda}-\lambda^{2} x^{+} x^{-}+\int^{x^{+}} d y^{+} \int^{y^{+}} d z^{+} \sum_{j=1}^{N} \partial_{+} a^{+}\left(z^{+}\right) \partial_{+} a^{+}\left(z^{+}\right) \\
& \quad+\int^{x^{-}} d y^{-} \int^{y^{-}} d z^{+} \sum_{j=1}^{N} \partial_{-} a^{-}\left(z^{-}\right) \partial_{-} a^{-}\left(z^{-}\right) .
\end{aligned}
$$

Here we have fixed the residual gauge symmetry of reparametrization by choosing the condition $\phi-\rho=0$, which is given by a sum of holomorphic function $w^{+}\left(x^{+}\right)$and anti-holomorphic function $w^{-}\left(x^{-}\right)$in general : $\phi\left(x^{+}, x^{-}\right)-\rho\left(x^{+}, x^{-}\right)=w^{+}\left(x^{+}\right)+w^{-}\left(x^{-}\right)$.

When we consider solutions with $a_{j}=0$, a special solution describing a dilaton vacuum is given by,

$$
\mathrm{e}^{-2 \phi}=\mathrm{e}^{-2 \rho}=-\lambda^{2} x^{+} x^{-} .
$$


One may get more general solutions,

$$
\mathrm{e}^{-2 \phi}=\mathrm{e}^{-2 \rho}=\frac{M}{\lambda}-\lambda^{2} x^{+} x^{-} .
$$

Here $\frac{M}{\lambda}$ is an integration constant. The solutions (148) tell that the metric has a singularity when $\frac{M}{\lambda}-\lambda^{2} x^{+} x^{-}=0$ and there appear horizons $x^{+} x^{-}=0$. Therefore, the solutions (148) describe black holes. The structure of space-time is similar to that of the $4 \mathrm{~d}$ Schwarzschild black holes.

The solutions (146) can also describe matter collapsing into black holes. As an example, we can consider a shock wave which is given by $\sum_{j=1}^{N} \partial_{ \pm} a_{j} \partial_{ \pm} a_{j}=a \delta\left(x^{+}-x_{0}^{+}\right)$. Then the solution is given by,

$$
\begin{aligned}
& x^{+}<x_{0}^{+}, \quad \mathrm{e}^{-2 \phi}=\mathrm{e}^{-2 \rho}=\frac{M}{\lambda}-\lambda^{2} x^{+} x^{-}, \\
& x^{+}>x_{0}^{+}, \quad \mathrm{e}^{-2 \phi} \quad=\mathrm{e}^{-2 \rho}=\frac{M}{\lambda}+a x_{0}^{+}-\lambda^{2} x^{+}\left(x^{-}+\frac{a}{\lambda^{2}}\right) .
\end{aligned}
$$

The event horizon when $x^{+}>x_{0}^{+}$is given by $x^{+}\left(x^{-}+\frac{a}{\lambda^{2}}\right)=0$. Note that the past horizon, where $x^{+}=-\frac{a}{\lambda^{2}}$, is shifted by the shock wave.

In the original paper by CGHS, they found that the Hawking radiation and the back reaction of the metric can be described by adding correction terms to the classical action. These terms come from the minimal scalar conformal anomaly (first term in (103)).

$$
W=-\frac{1}{2} \int d^{2} x \sqrt{-g} \frac{N}{48 \pi} R \frac{1}{\Delta} R
$$

However, they break the solvability in the classical CGHS model. Subsequently, Russo, Susskind and Thorlacius [83] added a dilaton dependent term in (114) by hands. That makes the semi-classical theory solvable. As shown in Eq. (103] [59], such term as in RST model can arise from the conformal anomaly for dilaton coupled spinors. CGHS and RST models initiated a lot of activity. Some other models which mainly represent their modifications and quantization of such models have been discussed in refs. $84,85,86,38,87,88,89,90$ (for a review and more complete list of refs., see [91]).

In 92], another solvable models which also represent some modification of CGHS theory are proposed. The starting classical action is given by

$$
S_{c}=\frac{1}{2 \pi} \int d^{2} x \sqrt{-g}\left\{\mathrm{e}^{-2 \phi}\left(R+4(\nabla \phi)^{2}+4 \lambda^{2}\right)-\frac{\mathrm{e}^{a \phi}}{g_{A}^{2}} F^{2}-\sum_{j=1}^{N} i \bar{\Psi}_{j} \gamma^{\mu}\left(D_{\mu}-i A_{\mu}\right) \Psi_{j}\right\} .
$$

Here $\phi$ is a dilaton field and $\Psi_{j}=\left(\begin{array}{c}\psi_{j} \\ 0\end{array}\right)$ 's are $N$ left-handed complex fermions. $g_{A}$ is a $U(1)$ electromagnetic gauge coupling constant and $D_{\mu}$ is a covariant derivative. If we fix $a=-2$, this action, except the fermion part, describes an effective field theory derived from string theory [36]. In ref. [92], it was chosen $a=2$, which allows us to exactly solve this model classically. In fact, when $a=-2$, the general solutions of effective equations are given by,

$$
\begin{aligned}
& \psi_{j}=\psi_{j}\left(x^{+}\right), \quad A_{+}=-\frac{g_{A}^{2}}{4} x^{-} \int^{x^{+}} d y^{+} \sum_{j=1}^{N} \psi_{j}^{*}\left(y^{+}\right) \psi_{j}\left(y^{+}\right) \\
& \mathrm{e}^{-2 \phi}=\mathrm{e}^{-2 \rho}=\frac{M}{\lambda}-\lambda^{2} x^{+} x^{-}+\frac{g_{A}^{2}}{8} x^{-} \int^{x^{+}} d y^{+}\left(\int^{y^{+}} d z^{+} \sum_{j=1}^{N} \psi_{j}^{*}\left(z^{+}\right) \psi_{j}\left(z^{+}\right)\right)^{2}
\end{aligned}
$$




$$
-\frac{i}{4} \int^{x^{+}} d y^{+} \int^{y^{+}} d z^{+} \sum_{j=1}^{N}\left(\psi_{j}^{*}\left(z^{+}\right) \partial_{+} \psi_{j}\left(z^{+}\right)-\partial_{+} \psi_{j}^{*}\left(z^{+}\right) \psi_{j}\left(z^{+}\right)\right) .
$$

A special solution with $\psi_{j}=0$ describes the dilaton vacuum in (147). And as more general solutions, we find

$$
F_{+-}=C \quad \text { (constant) }, \quad \mathrm{e}^{-2 \phi}=\mathrm{e}^{-2 \rho}=\frac{M}{\lambda}-\tilde{\lambda}^{2} x^{+} x^{-} .
$$

Here $\frac{M}{\lambda}$ is an integration constant and $\tilde{\lambda}$ is defined by $\tilde{\lambda}^{2} \equiv \lambda^{2}-\frac{2 C^{2}}{g_{A}^{2}}$. The metric has a singularity when $\frac{M}{\lambda}-\tilde{\lambda}^{2} x^{+} x^{-}=0$ and there appear horizons $x^{+} x^{-}=0$. Therefore, if $C \neq 0$, the solutions describe charged black holes. The structure of space-time is similar to that of the Schwarzschild black holes and simple in contrast to the Reissner-Nordstrøm black hole solutions in four dimensions. Note that the singularity vanishes when $\tilde{\lambda}^{2}=0$ when we fix $M$ to be finite. This solution could be a natural analogue of the extremal Reissner-Nordstrøm black hole solution. In fact, as the temperature $T$ is given by,

$$
T=\frac{\tilde{\lambda}}{2 \pi}
$$

the extremal solutions, which correspond to $\tilde{\lambda}^{2}=0$, have a vanishing temperature.

The solutions (152) can also describe charged matter (chiral fermions) collapsing into black holes. As an example, we can consider a charged shock wave which is given by, $\frac{i}{4} \sum_{j=1}^{N}\left(\psi_{j}^{*}\left(x^{+}\right) \partial_{+} \psi_{j}\left(x^{+}\right)-\right.$ $\left.\partial_{+} \psi_{j}^{*}\left(x^{+}\right) \psi_{j}\left(x^{+}\right)\right)=a \delta\left(x^{+}-x_{0}^{+}\right)$and $\frac{g_{A}^{2}}{4} \sum_{j=1}^{N} \psi_{j}^{*}\left(x^{+}\right) \psi_{j}\left(x^{+}\right)=b \delta\left(x^{+}-x_{0}^{+}\right)$. Then in the solution, not only future horizon corresponding to $x^{-}=-\frac{2 B x_{0}^{+}}{g_{A}^{2} \tilde{\lambda}^{\prime 2}}$, but past horizon, where $x^{+}=-\frac{a}{\lambda^{\prime 2}}$, is shifted by the shock wave. Here $\tilde{\lambda}^{\prime}$ and $B$ are defined by $\tilde{\lambda}^{\prime 2} \equiv \lambda^{2}-\frac{2(C+b)^{2}}{g_{A}^{2}}$ and $B \equiv\left(2 C b+b^{2}\right)$. In the original paper by 't Hooft 80], the shift of only future horizon was discussed in four dimensional black holes. The shift of the past horizon would suggest that charged particles collapsing into Reissner-Nordstrøm black hole in four dimensions could shift the past horizon.

Now, since we have massless fermions, the terms which come from the chiral anomaly should be also added to the quantum correction besides the terms coming from the conformal anomaly. Furthermore, by adding the contribution which corresponds to the term in RST model [83], we find the following total quantum action $S_{q}$ :

$$
S_{q}=S_{c}-\frac{N}{96} \frac{1}{2 \pi} \int d^{2} x \sqrt{-g}\left\{R \frac{1}{\Delta} R+\phi R\right\}+\frac{N}{24} \frac{1}{2 \pi} \int d^{2} x\left(\epsilon^{\mu \nu} F_{\mu \nu}\right) \frac{1}{\Delta}\left(\epsilon^{\mu \nu} F_{\mu \nu}\right) .
$$

The above model can be also solved in the same way as RST model. Of course, there are many more BH models which are not solvable. Their study is extremely difficult even technically.

Similarly, one can discuss other $2 \mathrm{~d}$ and $4 \mathrm{~d}$ BHs with Hawking radiation (for reviews on Hawking radiation see [91, 93]). As the presentation is more-less standard we finish this section by last example of supersymmetric BHs which are not exactly solvable.

In refs. [72, 59], the Hawking radiation of the supersymmetric extension of CGHS model 94 has been investigated. We discuss the case that dilaton function is given by $f(\phi)=\mathrm{e}^{\alpha \phi}$ in (142) in the previous chapter. The Hawking radiation can be obtained by substituting the classical black hole solution which appeared in the original CGHS model [35]

$$
\rho=-\frac{1}{2} \ln \left(1+\frac{M}{\lambda} \mathrm{e}^{\lambda\left(\sigma^{-}-\sigma^{+}\right)}\right), \quad \phi=-\frac{1}{2} \ln \left(\frac{M}{\lambda}+\mathrm{e}^{\lambda\left(\sigma^{+}-\sigma^{-}\right)}\right)
$$


(where $M$ is the mass of the black hole and we used asymptotic flat coordinates) into the quantum part of the energy momentum tensor. Then we find that when $\sigma^{+} \rightarrow+\infty$, the energy momentum tensor behaves as

$$
T_{+-}^{q} \rightarrow 0, \quad T_{ \pm \pm}^{q} \rightarrow \frac{N \lambda^{2}}{16} \alpha^{2}+t^{ \pm}\left(\sigma^{ \pm}\right) .
$$

Here $t^{ \pm}\left(\sigma^{ \pm}\right)$is a function which is determined by the boundary condition. In order to evaluate $t^{ \pm}\left(\sigma^{ \pm}\right)$, we impose a boundary condition that there is no incoming energy. This condition requires that $T_{++}^{q}$ should vanish at the past null infinity $\left(\sigma^{-} \rightarrow-\infty\right)$ and if we assume $t^{-}\left(\sigma^{-}\right)$is black hole mass independent, $T_{--}^{q}$ should also vanish at the past horizon $\left(\sigma^{+} \rightarrow-\infty\right)$ after taking $M \rightarrow 0$ limit. Then we find

$$
T_{--}^{q} \rightarrow 0
$$

at the future null infinity $\left(\sigma^{+} \rightarrow+\infty\right)$. Eqs.(157) and (158) might tell that there is no Hawking radiation in the dilatonic supergravity model under discussion when quantum back-reaction of matter supermultiplet in large- $N$ approach is taken into account as in [72]. (That indicates that above black hole is extremal one). From another side since we work in strong coupling regime it could be that new methods to study the Hawking radiation should be developed. This follows from the fact that despite various efforts there are still many unclear points related with Hawking radiation [95, 96, 97, 98, 99, 94, 100]. Especially puzzling is its relation with information loss paradox.

\subsection{Thermodynamics of Schwarzschild-de Sitter black holes}

The strong analogy between thermodynamics and BHs is known for quite a long time [101, 102]. One of its bright manifestations is related with Bekenstein-Hawking entropy [103, 79 which is known to be proportional to the surface area of $\mathrm{BH}$ horizon. Despite numerous attempts varying from strings 104 105, 106, 107 (for a review and list of refs. see 108), three-dimensional gravity 109, 110 including higher derivative terms 111, induced gravity 112], etc. the derivation of Bekenstein-Hawking entropy from statistical mechanics is not yet completely understood. Most probably, it is expected to get the complete understanding from string or M-theory. Our purpose in this section will be more modest than explanation of the origin of BH entropy. We are going to calculate quantum corrections to some classes of BHs semiclassically, using effective action obtained in previous chapter.

In 113], the quantum corrections to thermodynamics (and geometry) of S(A)dS BHs have been discussed by using large $N$ anomaly induced effective action for dilaton coupled matter (scalars and spinors). It is found the temperature, mass and entropy with account of quantum effects for multiply horizon SdS BH and SAdS BH. That also gives the corresponding expressions for their limits: Schwarzschild and de Sitter spaces. Note that such BHs are not dilatonic ones. Nevertheless, we develop the picture describing the same objects as $2 \mathrm{~d}$ dilatonic BHs. Hence, they again have the relation to dilatonic gravity.

We will start from the action of Einstein gravity with $N$ minimal real scalars $\chi_{i}$ and $M$ Majorana fermions $\psi_{i}$ :

$$
S_{4 d}=-\frac{1}{16 \pi G} \int d^{4} x \sqrt{-g_{(4)}}\left(R^{(4)}-2 \Lambda\right)+\int d^{4} x \sqrt{-g_{(4)}}\left(\frac{1}{2} \sum_{i=1}^{N} g_{(4)}^{\alpha \beta} \partial_{\alpha} \chi_{i} \partial_{\beta} \chi_{i}+\sum_{i=1}^{M} \bar{\psi}_{i} \gamma^{\mu} \nabla_{\mu} \psi_{i}\right)
$$


where $N$ and $M$ are considered to be large, $N, M \gg 1, G$ and $\Lambda$ are gravitational and cosmological constants, respectively. The convenient choice for the spherically symmetric spacetime is the following one

$$
d s^{2}=g_{\mu \nu} d x^{\mu} d x^{\nu}+e^{-2 \phi} d \Omega
$$

where $\mu, \nu=0,1, g_{\mu \nu}$ and $\phi$ depend only on $x^{0}, x^{1}$ and $d \Omega$ corresponds to the two-dimensional sphere. Then the action (159), reduced according to (160) takes the form

$$
S_{\text {red }}=\int d^{2} x \sqrt{-g} \mathrm{e}^{-2 \phi}\left[-\frac{1}{16 \pi G}\left\{R+2(\nabla \phi)^{2}-2 \Lambda+2 \mathrm{e}^{2 \phi}\right\}+\frac{1}{2} \sum_{i=1}^{N}\left(\nabla \chi_{i}\right)^{2}+\sum_{i=1}^{2 M} \bar{\psi}_{i} \gamma^{\mu} \nabla_{\mu} \psi_{i}\right]
$$

Working in large $N$ and $s$-wave approximation and using $2 \mathrm{~d}$ conformal anomaly for dilaton coupled scalar, calculated in [14] (see also [47, 51]) one can find the anomaly induced effective action [50, 46, 72] from Eqs. (103) and (113) by choosing $f(\phi)=\mathrm{e}^{-2 \phi}$. The conformally invariant functional can be found (see (105)) as some expansion of Schwinger-DeWitt type [57] keeping only the leading term. Then, the effective action may be written in the following form [46, 72, 57]

$$
W=-\frac{1}{8 \pi} \int d^{2} x \sqrt{-g}\left[\frac{N+M}{12} R \frac{1}{\Delta} R-N \nabla^{\lambda} \phi \nabla_{\lambda} \phi \frac{1}{\Delta} R+\left(N+\frac{2 M}{3}\right) \phi R+2 N \ln \mu_{0}^{2} \nabla^{\lambda} \phi \nabla_{\lambda} \phi\right],
$$

where $\mu_{0}^{2}$ is a dimensional parameter.

In the conformal gauge (145), it is convenient to change the radial coordinate $r$ by the new coordinate $x$

$$
x=\mathrm{e}^{-\phi},
$$

which corresponds to the usual coordinate choice in the Schwarzschild metric:

$$
d s^{2}=-\mathrm{e}^{2 \rho} d t^{2}+\mathrm{e}^{2 \sigma} d x^{2}+x^{2} d \Omega^{2}, \quad \mathrm{e}^{\sigma}=-\mathrm{e}^{\rho+\phi}\left(\frac{d \phi}{d r}\right)^{-1} .
$$

Note that there were also attempts in refs. 1114, 115, 46, 72, 57, 116 to apply such EA (usually without logarithmic term and only for scalars) for quantum considerations around BHs (for calculation of semiclassical stress tensor with dilaton, see refs. 117, 118]).

First we consider the case $\Lambda=0$. In the classical limit $(N \rightarrow 0)$, we obtain, of course, the Schwarzschild black hole as solution of classical equations of motion:

$$
\mathrm{e}^{2 \rho}=\mathrm{e}^{2 \rho_{0}}, \quad \mathrm{e}^{2 \sigma}=\mathrm{e}^{2 \sigma_{0}}, \quad \mathrm{e}^{2 \rho_{0}}=\mathrm{e}^{-2 \sigma_{0}}=1-\frac{\mu}{x} .
$$

Here $\mu=2 G M_{\mathrm{BH}}$ and $M_{\mathrm{BH}}$ is the black hole mass. We now consider the quantum corrections by regarding $G N$ is small and assuming

$$
\rho=\rho_{0}+G N \Delta_{\rho}, \quad \sigma=\sigma_{0}+G N \Delta_{\sigma}\left(\sigma_{0}=-\rho_{0}\right) .
$$

Then from effective equations of motion we find that $\Delta_{\sigma}$ behaves near the classical horizon $x \sim \mu$, as

$$
\Delta_{\sigma} \sim \frac{1}{3(x-\mu)}\left[\left(\frac{A}{8 \mu}+6 t_{0} \mu\right) \ln (x-\mu)+\text { regular terms }\right] .
$$


Here, $t_{0}$ is a constant which is determined by the initial conditions and $A \equiv \frac{N+M}{N}, B \equiv \frac{N+\frac{2 M}{3}}{N}$. The singularity coming from $\ln (x-\mu)$ vanishes if we choose (then $t_{0}$ here corresponds to the constants $C$ and $D$ of the integration in [116])

$$
t_{0}=-\frac{A}{48 \mu^{2}}
$$

In the choice of (168), one gets

$$
\begin{aligned}
\Delta_{\rho} & +\Delta_{\sigma}=A\left\{\frac{1}{8 x^{2}}+\frac{1}{6 \mu x}-\frac{1}{12 \mu^{2}} \ln \frac{x}{l}\right\} \\
+ & \frac{a+B-1}{2 x^{2}}-\frac{1}{4 x^{2}}-\frac{1}{2 \mu x}+\frac{1}{2}\left(\frac{1}{x^{2}}-\frac{1}{\mu^{2}}\right) \ln \left(1-\frac{\mu}{x}\right), \\
\Delta_{\sigma} & =\frac{1}{3(x-\mu)} \times\left\{\Delta_{S}+A\left(-\frac{1}{8 \mu} \ln \frac{x}{l}+\frac{1}{8 x}-\frac{7 \mu}{16 x^{2}}\right)+\frac{a-B+1}{2}\left(\frac{3}{x}-\frac{3 \mu}{2 x^{2}}\right)\right. \\
& \left.-\frac{3 B \mu}{4 x^{2}}-\frac{3 \mu}{4}\left(\frac{1}{x}-\frac{1}{\mu}\right)^{2} \ln \left(1-\frac{\mu}{x}\right)-\frac{3}{4 x}+\frac{3 \mu}{8 x^{2}}+\frac{3 x}{2 \mu^{2}}\right\} .
\end{aligned}
$$

Here parameters $l$ and $\Delta_{S}$ coming from the constants of the integration. We now assume the radius $L$ of the universe is large $L \gg \mu$ but finite and we require $\Delta_{\rho}=\Delta_{\sigma}=0$ when $x=L$. Then we find

$$
l=L, \quad \Delta_{S}=-\frac{3 L}{2 \mu^{2}} .
$$

Near the classical horizon, $\Delta_{\rho}+\Delta_{\sigma}$ and the scalar curvature are regular. The horizon defined by $\mathrm{e}^{2 \rho}=0$, which corresponds to $x=\mu$ in the classical limit is given by

$$
0 \sim \frac{1}{\mu}\left(x-\mu+2 G N C_{S}\right)
$$

Then the entropy, which is defined by the area of horizon divided by $4 G$, is

$$
S \sim \frac{\pi \mu^{2}}{G}-\pi N\left(-\frac{4 \mu \Delta_{S}}{3}+\frac{A}{6} \ln \frac{\mu}{l}+\frac{5 A}{12}-a+2 B-\frac{5}{2}\right) .
$$

The second term in (172) corresponds to the quantum correction. From the behavior of the metric near the horizon $x=\mu-2 G N C_{S}$, we find the temperature $T$ (in the following, we put the Boltzmann constant $k$ to be unity, $k=1$ ) is given by

$$
T \sim \frac{1}{4 \pi \mu}\left[1+G N\left\{-\frac{2}{3 \mu} \Delta_{S}+\frac{A}{12 \mu^{2}}\left(-1+2 \ln \frac{\mu}{l}\right)-\frac{a}{\mu^{2}}+\frac{B}{2 \mu^{2}}\right\}\right] .
$$

The second term corresponds to the quantum correction. We now consider the thermodynamical mass $E$, which is defined by

$$
d E=T d S
$$

Using the parameter $\mu,(174)$ can be rewritten as

$$
E=\int d \mu T \frac{d S}{d \mu}=\frac{\mu}{2 G}+N\left(-\frac{L}{2 \mu^{2}}-\frac{A}{12 \mu} \ln \frac{\mu}{L}+\frac{a}{2 \mu}+\frac{B}{4 \mu}\right) .
$$

The first term expresses the usual classical mass $M_{\mathrm{BH}}$ since $\mu=2 G M_{\mathrm{BH}}$ and the second term is due to the quantum effects. Notice the regularization scheme dependence via the presence of parameter a in above expressions. It may be fixed by the choice of some physical regularization. The qualitative structure of 
the entropy (172), the temperature (173) and the energy (mass) (175) are similar to the corresponding quantities found in 116] for pure scalar.

Let us turn now to $2 \mathrm{~d}$ formulation of above results. The classical black hole solution (165) can be regarded as a purely two dimensional object if we start with the reduced action (161). The thermodynamical quantities as the energy and entropy for two dimensional charged black hole with dilaton are evaluated on the classical and one-loop levels in [57, 119]. In [119], the boundary of the universe is introduced at the radius $r=L$. If there is a boundary, we need to add the boundary terms to the action in order that the variation with respect to the metric should be well-defined. By including the boundary term, the formula for the energy (mass) of the black hole with one-loop quantum correction was derived in 119] as follows,

$$
E(\mu, L)=-\frac{\mathrm{e}^{\lambda(L)}}{G} g^{\frac{1}{2}}(L) D^{\prime}(\phi(L))-\frac{\hbar}{3} \mathrm{e}^{\lambda_{C L}(L)} g_{C L}^{-\frac{1}{2}}(L) g_{C L}^{\prime}(L)-\frac{c \hbar}{6} \mathrm{e}^{\lambda_{C L}(L)} g_{C L}^{\frac{1}{2}}(L) \phi^{\prime}(L) .
$$

Here $^{\prime}=\frac{d}{d x}$. The quantities appeared in (176) have the following correspondence with the quantities here,

$$
g(x)=\mathrm{e}^{2 \rho(x)}, \quad \mathrm{e}^{-\lambda(x)}, \quad D(\phi(x))=\frac{\mathrm{e}^{-2 \phi(x)}}{2}=\frac{x^{2}}{2} .
$$

Since only the quantum correction from the one scalar field was evaluated in [119, we need to do the replacement $\hbar \rightarrow N A$ and $c \rightarrow \frac{12 B}{A}$. We should note that we usually choose $\hbar=1$. In the expression (176), the quantities with suffices $C L$ correspond to the classical ones. If we choose a proper boundary condition, we find

$$
g(L)=g_{C L}(L)=1-\frac{\mu}{L}, \quad \Lambda(L)=\Lambda_{C L}(L)=0
$$

Therefore

$$
E(\mu, L)=-\frac{L}{G}\left(1-\frac{\mu}{L}\right)^{\frac{1}{2}}-\frac{N A \mu}{3 L^{2}}\left(1-\frac{\mu}{L}\right)^{-\frac{1}{2}}+\frac{2 N B}{L}\left(1-\frac{\mu}{L}\right)^{\frac{1}{2}} .
$$

Since the energy 179 ) diverges in the limit $L \rightarrow+\infty$, we need to subtract $E(0, L)$ before taking the limit of $L \rightarrow+\infty$. Then we obtain

$$
E_{\text {sub }} \equiv \lim _{L \rightarrow+\infty}(E(\mu, L)-E(0, L))=\frac{\mu}{2 G}
$$

which is nothing but the classical black hole mass. This tells that there is no quantum correction for the mass. Even if the black hole is purely two dimensional one (but with dilaton), the definition of the temperature is not changed and one gets (173). Then using the energy (180), the temperature (173) and the definition of the entropy (174), we obtain the following expression of the entropy

$$
\begin{aligned}
S= & \int \frac{d E}{T}=\int \frac{d \mu}{T(\mu)} \frac{d E}{d \mu} \\
= & \frac{\pi \mu^{2}}{G}-2 \pi N\left\{-\frac{L}{\mu}+\left\{-\frac{A}{12}-a+\frac{B}{2}\right) \ln \frac{\mu}{l}+\frac{A}{12}\left(\ln \frac{\mu}{L}\right)^{2}+c\right\} \\
& \left.+\mathcal{O}\left(L^{-1}\right)+\mathcal{O}(G N)^{2}\right) .
\end{aligned}
$$

Here $c$ is the constant of the integration. The classical part coincides with the usual Bekenstein-Hawking entropy when we regard the black hole as the four dimensional object. 
Hence we calculated quantum corrections to simplest black hole thermodynamical quantities. The result is actually obtained for two objects: $4 \mathrm{~d} \mathrm{BH}$ and the same object described as $2 \mathrm{~d}$ dilatonic $\mathrm{BH}$. That shows remarkable property of $s$-wave EA that it could be applied to $4 \mathrm{~d}$ as well as to $2 \mathrm{~d}$ geometry (where it looks already as complete EA). It is not difficult to extend the discussion for other types of BHs.

We now consider more general Schwarzschild-(anti-)de Sitter black holes. Their convenient description is given in next section. In the classical limit $(N \rightarrow 0)$ with non-vanishing cosmological constant $\Lambda$, we obtain the usual Schwarzschild-(anti)de Sitter as solution of equations of motion

$$
\mathrm{e}^{2 \rho}=\mathrm{e}^{-2 \sigma}=\mathrm{e}^{2 \rho_{0}} \equiv 1-\frac{\mu}{x}-\frac{\Lambda}{3} x^{2}=-\frac{\Lambda}{3 x} \prod_{i=1}^{3}\left(x-x_{i}\right) .
$$

Here $\mu$ is a constant of the integration corresponding to the black hole mass $\left(\mu=2 G M_{\mathrm{BH}}\right)$. The parameters $x_{i}(i=1,2,3)$ are solutions of the equation $\mathrm{e}^{2 \rho_{0}}=0$. Among $x_{i}$ 's, two are real and positive if $\Lambda>0$ and $\mu^{2}<\frac{4}{9 \Lambda}$ and they correspond to black hole and cosmological horizons in the Schwarzschild-de Sitter black hole. On the other hand, only one is real if $\Lambda<0$. If we start from the reduced action (161), the classical solution (182) can be regarded to express the purely two dimensional Schwarzschild-(anti) de Sitter black holes with dilaton. Hence, we again obtain $2 \mathrm{~d}$ or $4 \mathrm{~d}$ formulation for such object. Note that reduction of SAdS BH may be understood as $2 \mathrm{~d}$ dilatonic AdS BH where quantum effects of dilaton were recently discussed in refs. 120, 121.

As in (166), we consider the quantum corrections regarding $G N$ as small. In a similar way as in the case of the Schwarzschild black hole, we obtain the following expressions for the entropy $S$ and the temperature $T$ :

$$
\begin{aligned}
S & \sim \pi x_{I}^{2}-4 \pi G N C_{I} x_{I} \\
T & \sim\left|\frac{\Lambda}{12 \pi}\left\{Y_{I}+\left(-6+\frac{2 Y_{I}}{x_{I}}\right) G N C_{I}-G N Y_{I} B_{I}\right\}\right| .
\end{aligned}
$$

The explicit forms of $B_{I}$ and $C_{I}$ are given in 113 , and

$$
Y_{i} \equiv \frac{1}{x_{i}} \prod_{i, j=1, i \neq j}^{3}\left(x_{j}-x_{i}\right), \quad a^{\prime} \equiv \ln \left(-\frac{\Lambda}{3}\right)+a .
$$

We now consider de Sitter space as a limit of $\mu \rightarrow 0$. Let us choose $x_{I}$ as a cosmological horizon : $x_{I}=h \equiv \sqrt{\frac{3}{\Lambda}}$. Then we find the expressions for the entropy $S$ and the temperature $T$ :

$$
\begin{aligned}
S & =\frac{3 \pi}{\Lambda}-2 \pi G N\left\{\tilde{\Delta}_{1} \sqrt{\frac{\Lambda}{3}}+\frac{A}{6}+a+1+2 \ln 2\right\} \\
T & =\frac{1}{2 \pi} \sqrt{\frac{\Lambda}{3}}+\frac{G N \Lambda}{12 \pi}\left\{\frac{\Lambda \tilde{\Delta}_{1}}{3}-2 \tilde{\Delta}_{0} \sqrt{\frac{3}{\Lambda}}+\left(\frac{A}{2}+2+2 \ln 2-B\right) \sqrt{\frac{\Lambda}{3}}\right\} .
\end{aligned}
$$

Here $\tilde{\Delta}_{0}$ and $\tilde{\Delta}_{1}$ are the constants of the integration. It is interesting to note that the expression for $S$ describes quantum corrections to the entropy of expanding inflationary Universe (as de Sitter space may be considered as such inflationary Universe). That gives new terms proportional to particles number as compare with classical entropy of expanding Universe discussed extensively in refs. 122, 123, 124]. 
Hence we found quantum corrections to the temperature and the entropy for $4 \mathrm{~d}$ de Sitter space (as the limit of SdS BH) as well as for SdS BH and SAdS BH. In the last case of SdS or SAdS BHs we also defined the quantum correction to the temperature of corresponding $2 \mathrm{~d}$ object (i.e. corresponding $\mathrm{BH}$ with dilaton). The calculation of $2 \mathrm{~d}$ quantum entropy is more difficult and cannot be done by using only $4 \mathrm{~d}$ point of view unlike the case of Schwarzschild BH. Similarly, one can calculate quantum corrections to other $\mathrm{BHs}$, for example, for charged $4 \mathrm{~d} \mathrm{BHs}$ it was done in ref. 125. Below we consider one more example of $2 \mathrm{~d}$ charged BHs.

In 119, it was investigated the one-loop quantum correction to the thermodynamical quantities in general two- dimensional charged black holes, whose special cases correspond to the dimensionally reduced higher dimensional black holes like $4 \mathrm{~d}$ charged spherically symmetric one or $3 \mathrm{~d}$ rotating BTZ one. The starting classical action is given by

$$
W\left[g_{\mu \nu}, \phi, A_{\mu}\right]=\frac{1}{2 G} \int d^{2} x \sqrt{-g}\left[D(\phi) R+\frac{1}{2} \partial_{\alpha} \phi \partial_{\beta} \phi+\frac{1}{l^{2}} V(\phi)-\frac{2 G}{4} Y(\phi) F^{\alpha \beta} F_{\alpha \beta}\right]
$$

where $A_{\mu}$ is the abelian gauge field and $F_{\mu \nu}=\partial_{\mu} A_{\nu}-\partial_{\nu} A_{\mu} . \quad D(\phi), V(\phi)$, and $Y(\phi)$ are arbitrary dilatonic functions. The free energy $F$ can be evaluated from the action $W$ after Wick-rotating to Euclidean signature $t \rightarrow i \tau$ by $F=\frac{1}{2 \pi \beta} W$ after the subtraction of the contribution from the adequate background geometry. Here $\bar{\beta}$ is the inverse of the temperature which is red-shifted at the boundary at $x=L \bar{\beta}=g^{\frac{1}{2}} \beta$. In order to well define the quantities, the space-time is assumed to be the disc, where the space coordinate $x$, corresponding the radial coordinate in the choice of the Schwarzschild like metric

$$
d s^{2}=g(x) d \tau^{2}+\mathrm{e}^{-\lambda(x)} g^{-1}(x) d x^{2},
$$

is defined between the outer horizon at $x=x_{+}$to the finite boundary at $x=L$. Other thermodynamical quantities like the entropy $S$ and the energy $E$ can be obtained by using the standard definitions in the thermodynamics:

$$
S=\bar{\beta}^{2} \frac{\partial F}{\partial \bar{\beta}}, \quad E=\frac{\partial(\bar{\beta} F)}{\partial \bar{\beta}} .
$$

In 119, the quantum corrections were included by adding the one-loop effective action $\Gamma$ to the classical action (185). $\Gamma$ is generated by the $2 \mathrm{~d}$ conformal anomaly as given in Eq. (103) or 113) :

$$
\Gamma=\frac{1}{12} \int d^{2} x \sqrt{-g}\left[a R \frac{1}{\triangle} R+b(\nabla \phi)^{2} \frac{1}{\triangle} R+c \phi R\right] .
$$

The parameter $a$ is chosen to be unity $a=1$ and $b$ and $c$ are arbitrary. Then the entropy $S$ is given by

$$
\begin{aligned}
S & =\frac{2 \pi}{G} D\left(\phi_{C L}\left(x_{+}\right)\right) \\
& -\frac{2 \pi \hbar}{6}\left[2 \psi\left(x_{+}\right)-c \phi_{C L}\left(x_{+}\right)-b \int_{x_{+}}^{L} d x \frac{\mathrm{e}^{-\lambda_{C L}(x)}}{g_{C L}(x)} \int_{x}^{L} d \bar{x} \mathrm{e}^{\lambda_{C L}(\bar{x})} \phi_{C L}^{\prime}(\bar{x})^{2} g_{C L}(\bar{x})\right],
\end{aligned}
$$

and the energy $E$ is given by Eq.(176). Here the classical quantities are notated by the suffices $C L$ and $\psi$ is defined when we choose the Euclidean metric in the following way:

$$
d s^{2}=\mathrm{e}^{-\psi}\left(z^{2} d \theta^{2}+d z^{2}\right)
$$


that is

$$
g(x) d t^{2}=\mathrm{e}^{-\psi} z^{2} d \theta^{2}, \quad g^{-1}(x) \mathrm{e}^{-2 \lambda(x)} d x^{2}=\mathrm{e}^{-\psi} d z^{2}
$$

We can regard the above $2 \mathrm{~d}$ model as $4 \mathrm{~d}$ spherically symmetric reduced model if we identify

$$
\phi=\frac{\sqrt{2} r}{l}, \quad D(\phi)=\frac{r^{2}}{2 l^{2}}, \quad V(\phi)-\frac{G l^{2} q^{2}}{Y(\phi)}=1-\frac{Q^{2}}{r^{2}} .
$$

Here $q$ corresponds to the charge in $2 \mathrm{~d}$ model. Then we obtain the following expressions for the above thermodynamical quantities:

$$
\begin{aligned}
& S \sim S_{C L}+2 \pi \beta_{C L} m\left(r_{+C L}\right)+\frac{2 \pi}{3}\left(1-\frac{r_{-C L}^{2}}{r_{+C L}^{2}}\right) \ln \left(\frac{L}{r_{+C L}-r_{+C L}}\right)+\frac{4 \pi}{3} \ln \left(\frac{r_{+C L}}{z_{0}}\right) \\
& E \sim M_{C L}+\frac{\hbar G l^{2} M_{C L}}{3 \beta_{C L}^{2}}(2 \ln (L)+1) .
\end{aligned}
$$

Here we assume that $L$ is large and neglect $\mathcal{O}\left(L^{-2}\right)$ terms. $m(r)$ expresses the quantum correction to the mass:

$$
m(r)=M(r)-M_{C L} \sim \frac{\hbar}{3 \beta_{C L}^{2}}\left[L+2 M_{C L} G l^{2} \ln (L)\right]
$$

and $z_{0}$ is the value of $z$ corresponding to $x=L$.

Thus, we presented the derivation of quantum corrections to thermodynamics of different $2 \mathrm{~d}$ and $4 \mathrm{~d}$ BHs using the effective action derived in previous chapter. There is no problem to extend such semiclassical investigation for other BH models. It is also interesting to note that some integrable dilatonic gravity models lead to the solutions similar to Nariai space [90] where again quantum corrections may be found.

\subsection{Anti-evaporation of multiply horizon black holes}

In [79], Hawking made one of the most striking discoveries of the history of black hole physics: the possibility for black holes to evaporate, as a result of particle creation. This effect — which is now called the Hawking radiation process - produced a deep impact in our understanding of quantum gravity.

There exists, however, an exotic class of black holes (see Ref. [102 for a review) which possesses a multiple horizon and for which the opposite effect might occur. It is interesting to investigate this possibility in detail. Consider a nearly degenerate Schwarzschild-de Sitter black hole (the so-called Nariai black hole [126]). The Schwarzschild-de Sitter black hole represents the neutral, static, spherically symmetric solution of Einstein's theory with a cosmological constant. The corresponding metric looks as follows

$$
d s^{2}=-V(\tau) d t^{2}+V^{-1}(\tau) d \tau^{2}+\tau^{2} d \Omega^{2}, \quad V(\tau)=1-\frac{2 \mu}{\tau}-\frac{\Lambda}{3} \tau^{3},
$$

where $\mu$ is the mass and $\tau$ the radius of the black hole, $d \Omega^{2}$ the metric corresponding to a unit two-sphere, and $\Lambda$ is the cosmological constant. It can be easily checked that the equation $V(\tau)=0$ has two positive roots, $\tau_{c}$ and $\tau_{b}$ (we set $\tau_{c}>\tau_{b}$ ). Here, $\tau_{c}$ and $\tau_{b}$ have the meaning of a cosmological and a black-hole horizon radius, respectively. In the degenerate case (which corresponds to a black hole of maximal mass) 
both radii coincide and the black hole is in thermal equilibrium. It is called a Nariai black hole. If we redefine new time and radial coordinates $\psi$ and $\chi$ by

$$
\tau=\frac{1}{\epsilon \sqrt{\Lambda}} \psi, \quad r=\frac{1}{\sqrt{\Lambda}}\left[1-\epsilon \cos \chi-\frac{1}{6} \epsilon^{2}\right],
$$

the Nariai limit is given by $\epsilon \rightarrow 0$. In the Nariai limit, the space-time has the topology of $S^{1} \times S^{2}$ and the metric is given by

$$
d s^{2}=\frac{1}{\Lambda}\left(\sin ^{2} \chi d \psi^{2}-d \chi^{2}-d \Omega^{2}\right) .
$$

Here coordinate $\chi$ has a period $\pi$. If we change the coordinates variables by

$$
r=\ln \tan \frac{\chi}{2}, \quad t=\frac{\psi}{4},
$$

we obtain

$$
d s^{2}=\frac{1}{\Lambda \cosh ^{2} r}\left(-d t^{2}+d r^{2}\right)+\frac{1}{\Lambda} d \Omega .
$$

This form corresponds to the conformal gauge in two dimensions.

There are in fact two opposite sources contributing to the thermal equilibrium, namely a radiation flux coming from the cosmological horizon and the Hawking evaporation originated at the black hole horizon. It is plausible that such a state might be unstable, since it could be affected by small perturbations of the geometry.

It was demonstrated in by Bousso and Hawking [114], that a nearly maximal (or nearly degenerated) Nariai black hole may not only evaporate — as shown in Ref. [79] - but also anti-evaporate [114]. In other words, Nariai black holes actually develop two perturbative modes: an evaporating one and an anti-evaporating one. The realization of this quantum process, carried out in Ref. [114], is based on the $s$-wave approximation by the spherical reduction and the $2 \mathrm{~d}$ conformal anomaly induced effective action due to $\mathrm{N}$ dilaton coupled scalars.

In the model of [114], the possibility of evaporation or anti-evaporation (i.e., increase in size) of a black hole of this kind is connected with the initial conditions chosen for the perturbations. The use of the commonly employed Hartle-Hawking no-boundary conditions 127 shows that such black holes will most likely evaporate.

The Nariai black holes are not asymptotically flat and they will never appear in the process of star collapse. Nevertheless, they could actually be present in the early inflationary universe. There may be at least two mechanisms to produce such multiple horizon BHs. First of all, they may appear via black holes pair creation [128] in the inflationary Universe. Second, it is possible the direct quantum generation of such objects 129] in effective dilatonic gravity models. In the model in Ref. 114], however, the primordial multiple horizon black holes should quickly evaporate, and it is very unlikely that they could be detected in the present universe.

However, a different model (with another matter content) for anti-evaporation of black holes has been proposed in Refs. 130, 131, in which these difficulties may be solved. In that model, the quantum effects of the conformally invariant matter have been taken into account. What is more interesting, this theory 
allows for the possibility of including not only scalar fields, but also fermionic and vector fields (typical of all grand unified theories, GUTs), whose classical action is

$$
S=\int d^{4} x \sqrt{-g_{(4)}}\left\{\frac{1}{2} \sum_{i=1}^{N}\left(g_{(4)}^{\alpha \beta} \partial_{\alpha} \chi_{i} \partial_{\beta} \chi_{i}+\frac{1}{6} R^{(4)} \chi_{i}^{2}\right)-\frac{1}{4} \sum_{j=1}^{N_{1}} F_{j \mu \nu} F_{j}^{\mu \nu}+\sum_{k=1}^{N_{1 / 2}} \bar{\psi}_{k} D \psi_{k}\right\}
$$

and its quantum correction $\Gamma$ is the sum of the conformal anomaly induced action $W$, as given by Eq. (220) $(f=1)$ and one corresponding to (125), and the action $\Gamma^{\prime}$ deduced from the Schwinger-de Witt type expansion :

$$
\begin{aligned}
\Gamma & =W+\Gamma^{\prime}, \\
W & =b \int d^{4} x \sqrt{-g} F \sigma+b^{\prime} \int d^{4} x \sqrt{-g}\left\{\sigma\left[2 \square^{2}+4 R^{\mu \nu} \nabla_{\mu} \nabla_{\nu}-\frac{4}{3} R \square+\frac{2}{3}\left(\nabla^{\mu} R\right) \nabla_{\mu}\right] \sigma\right. \\
& \left.+\left(G-\frac{2}{3} \square R\right) \sigma\right\}-\frac{b+b^{\prime}}{18} \int d^{4} x \sqrt{-g}[R-6 \square \sigma-6(\nabla \sigma)(\nabla \sigma)]^{2}, \\
\Gamma^{\prime} & =\int d^{4} x \sqrt{-g}\left\{\left[b F+b^{\prime} G+\frac{2 b}{3} \square R\right] \ln \frac{R}{\mu^{2}}\right\}+\mathcal{O}\left(R^{3}\right) .
\end{aligned}
$$

Here $b=\frac{\left(N+6 N_{1 / 2}+12 N_{1}\right)}{120(4 \pi)^{2}}, b^{\prime}=-\frac{\left(N+11 N_{1 / 2}+62 N_{1}\right)}{360(4 \pi)^{2}}, \mu$ is a mass-dimensional constant parameter, and we choose the spherically symmetric metric as

$$
d s^{2}=\mathrm{e}^{-2 \sigma}\left(\sum_{\alpha, \beta=0,1} g_{\alpha \beta} d x^{\alpha} d x^{\beta}+r_{0}^{2} d \Omega^{2}\right),
$$

where $d \Omega^{2}$ is the metric on the unit two-sphere. The parameter $r_{0}$ is introduced by hands. Note that the Schwinger-De Witt expansion is the corresponding power expansion with respect to the curvature. Having introduced the parameter $r_{0}$, the scalar curvature given by the metric $g_{\mu \nu}$ is of the order of $1 / r_{0}^{2}$ if there is no singularity, as in the Nariai black hole. Therefore, if we choose $r_{0}$ to be large, the Schwinger-de Witt type expansion becomes exact.

Solving the quantum effective equations of motion derived from 200 and (201), we can find the quantum analogue of the Nariai black hole, which has constant scalar curvature $R=R_{0}$ and radius $\mathrm{e}^{\sigma}=\mathrm{e}^{\sigma_{0}}$ :

$$
\begin{aligned}
R_{0}= & \left\{2+\left(\ln \left(\mu r_{0}\right)\right)^{-1}\left(\frac{2 b+3 b^{\prime}}{b}+\frac{9}{512 \pi^{2} b G \Lambda}\right)\right\}+\mathcal{O}\left(\left(\ln \left(\mu r_{0}\right)\right)^{-1}\right) \\
\sigma_{0}= & -\ln \left(\mu r_{0}\right)+\frac{1}{2} \ln \left(\frac{3 \mu^{2}}{2 \Lambda}\right) \\
& +\left(\ln \left(\mu r_{0}\right)\right)^{-1} \frac{\mu^{2}}{8 \Lambda}\left(\frac{2 b+3 b^{\prime}}{b}+\frac{9}{512 \pi^{2} b G \Lambda}\right)+\mathcal{O}\left(\left(\ln \left(\mu r_{0}\right)\right)^{-1}\right) .
\end{aligned}
$$

Here we assume $r_{0}$ to be large. Then the metric in (202) has the following form:

$$
d s^{2}=\mathrm{e}^{2 \sigma_{0}+2 \rho}\left(-d t^{2}+d r^{2}+r_{0}^{2} d \Omega^{2}\right), \quad \mathrm{e}^{2 \rho}=\mathrm{e}^{2 \rho_{0}} \equiv \frac{2 C}{R_{0}} \cdot \frac{1}{\cosh ^{2}(r \sqrt{C})} .
$$

Here $C$ is a constant of integration.

Furthermore, we can find the perturbation around the solution:

$$
\rho=\rho_{0}+\epsilon R(t, r), \quad \sigma=\sigma_{0}+\epsilon S(t, r) .
$$


If we assume $R$ and $S$ are the eigenfunctions with eigenvalue $A$ of the Laplacian $\Delta$ in the two-dimensional hyperboloid, which corresponds to the subspace in the Nariai black hole, given by radial and time coordinates

$$
\Delta=\cosh ^{2}(r \sqrt{C}) \partial_{+} \partial_{-}
$$

the perturbative equations of motion become two linear algebraic equations. From the condition that the two equations have non-trivial solutions, the eigenvalue $A$ can be determined.

The fate of the perturbed black hole is governed by the eigenvalue $A$ of the Laplacian $\Delta$. We now consider the following function as an eigenfunction of $\Delta$ in 206):

$$
f_{A}(t, r)=\cosh t \alpha \sqrt{C} \cosh ^{\alpha} r \sqrt{C}, \quad A \equiv \frac{\alpha(\alpha-1) C}{4} .
$$

Note that there is one to one correspondence between $A$ and $\alpha$ if we restrict $A>0$ and $\alpha<0$. The horizon is given by the condition

$$
\nabla \sigma \cdot \nabla \sigma=0
$$

Substituting (207) into (208), we find the horizon is given by $r=\alpha t$. Therefore on the horizon, we obtain

$$
S(t, r(t))=Q \cosh ^{1+\alpha} t \alpha \sqrt{C}
$$

This tells that the system is unstable if there is a solution $0>\alpha>-1$, i.e., $0<A<\frac{C}{2}$. On the other hand, the perturbation becomes stable if there is a solution where $\alpha<-1$, i.e., $A>\frac{C}{2}$. The radius of the horizon $r_{h}$ is given by

$$
r_{h}=\mathrm{e}^{\sigma}=\mathrm{e}^{\sigma_{0}+\epsilon S(t, r(t))} .
$$

Let the initial perturbation is negative $Q<0$. Then the radius shrinks monotonically, i.e., the black hole evaporates in case of $0>\alpha>-1$. On the other hand, the radius increases in time and approaches to the Nariai limit asymptotically $S(t, r(t)) \rightarrow Q \mathrm{e}^{(1+\alpha) t|\alpha| \sqrt{C}}$ in case of $\alpha<-1$. The latter case corresponds to the anti-evaporation of black hole observed by Bousso and Hawking [114. We should be more careful in the case of $A=\frac{C}{2}$. When $A=\frac{C}{2}, f_{A}(r, t)$ is, in general, given by

$$
f_{A}(r, t)=\left\{\frac{\cosh ((t+a) \sqrt{C})}{\cosh (r \sqrt{C})}+\sinh (b \sqrt{C}) \tanh (r \sqrt{C})\right\} .
$$

Then the condition (208) gives $t+a=\mp(r-b)$. Therefore on the horizon, we obtain $S(t, r(t))=Q \cosh b$. This is a constant, that is, there does not occur evaporation nor anti-evaporation. The radius of the horizon does not develop in time. In classical case, we find $A=\frac{C}{2}$. Therefore the horizon does not develop in time and the black hole does not evaporate nor anti-evaporate. The result is, of course, consistent with that of 114 .

The eigenvalue $A$ is now explicitly given by

$$
A=\frac{C}{2}+\left(\ln \left(\mu r_{0}\right)\right)^{-1} a_{1}, \quad a_{1}=0, \text { or }-\frac{\left(b+b^{\prime}\right) C}{8 b^{\prime}}
$$

Here we neglect the term of $\mathcal{O}\left(\left(\ln \left(\mu r_{0}\right)\right)^{-2}\right)$ since we assume $r_{0}$ is large. In the first solution for $a_{1}$, the horizon does not develop in time and we would need the analysis of the higher order of $\left(\ln \left(\mu r_{0}\right)\right)^{-1}$. An 
important thing is the second solution is positive when

$$
2 N+7 N_{1 / 2}>26 N_{1}
$$

When $a_{1}$ is positive, $A>\frac{C}{2}$, i.e., there occurs anti-evaporation. Since the equations of motion in the model by Bousso and Hawking contain only second order derivatives, anti-evaporation is excluded there by the no-boundary condition of Hartle and Hawking. The quantum effective equations of motion given by (200) and (201), however, contain fourth order derivatives and there the anti-evaporation phenomenon can be consistent with the no-boundary condition.

For the usual SO(10) GUT, which would be a typical elementary particle physics model in the early universe, it is shown that anti-evaporation may occur there only in its SUSY version [131. One may conclude that the existence of primordial black holes in the present universe might be considered as an indirect evidence for supersymmetry.

It could be really interesting to investigate the phenomena of the anti-evaporation in other types of BHs. The natural candidate to think about is charged BH. Working in large $N$ approach for dilaton coupled quantum scalars we present below some quantum properties of $2 \mathrm{~d}$ charged BHs. The corresponding classical solutions have been found in [36] (see also ref. 132]). They may be considered as some compactifications of Type II string solutions. Moreover, very naturally they may be considered as $2 \mathrm{~d}$ analog of Reissner-Nordström (RN) charged 4d BH.

We start from the action which has been considered by McGuigan, Nappi and Yost in ref. 36]. This action follows from compactification of heterotic string theory:

$$
S=\frac{1}{16 \pi G} \int d^{2} x \sqrt{-g} \mathrm{e}^{-2 \phi}\left(R+4(\nabla \phi)^{2}+4 \lambda^{2}-4 F_{\mu \nu}^{2}\right) .
$$

Note that above action has the form typical for $4 \mathrm{~d}$ or $5 \mathrm{~d}$ Einstein-Maxwell theory spherically reduced to two dimensions. Hence, it can be considered as toy model to describe $4 \mathrm{~d}$ or $5 \mathrm{~d}$ BH with spherical reduction.

It is remarkable that action (214) has the classical solutions which correspond to the $2 \mathrm{~d}$ charged black hole ( $2 \mathrm{~d}$ analogue of Reissner-Nordström black hole) with multiple horizon. In the explicit form its metric and dilaton look like 36,132$]$

$$
\begin{aligned}
& d s^{2}=-\left(1-2 m \mathrm{e}^{-2 \lambda x}+q^{2} \mathrm{e}^{-4 \lambda x}\right) d t^{2}+\left(1-2 m \mathrm{e}^{-2 \lambda x}+q^{2} \mathrm{e}^{-4 \lambda x}\right)^{-1} d x^{2} \\
& \mathrm{e}^{-2\left(\phi-\phi_{0}\right)}=\mathrm{e}^{2 \lambda x} .
\end{aligned}
$$

Here $q$ and $m$ are parameters related to the charge and the mass of $\mathrm{BH}$, respectively. The extremal solution is given by putting $q^{2}=m^{2}$. If we define new coordinates $r$ and $\tau$ by

$$
\mathrm{e}^{-2 \lambda x}=\frac{m+\epsilon \tanh 2 \lambda r}{q^{2}}, \quad t=\frac{q}{\epsilon} \tau, \quad \epsilon^{2} \equiv m^{2}-q^{2},
$$

$r \rightarrow+\infty$ corresponds to outer horizon and $r \rightarrow-\infty$ corresponds to inner horizon. Taking the limit $\epsilon \rightarrow 0$, we obtain

$$
d s^{2}=\frac{1}{\cosh ^{2} \lambda r}\left(d \tau^{2}-d r^{2}\right), \quad \mathrm{e}^{-2\left(\phi-\phi_{0}\right)}=\frac{1}{q} .
$$


Note that the dilaton field becomes a constant in the limit.

We will discuss now the quantum corrections induced by $N$ free conformally invariant dilaton coupled scalars $f_{i}$ (no background scalars $f_{i}$ ):

$$
S^{f}=-\frac{1}{2} \int d^{2} x \sqrt{-g} \mathrm{e}^{-2 \phi} \sum_{i=1}^{N}\left(\nabla f_{i}\right)^{2} .
$$

Using the effective action induced by the conformal anomaly plus conformally invariant part calculated in the Schwinger-De Witt type expansion, we find the quantum analogue of the extremal solution in (217), where $\phi$ and the scalar curvature are constant:

$$
\phi=\phi_{0}, \quad R=-2 \mathrm{e}^{2 \rho} \partial_{r}^{2} \rho=R_{0} \equiv-\frac{8 \lambda^{2} \mathrm{e}^{-2 \phi_{0}}}{\mathrm{e}^{-2 \phi_{0}}-\frac{2 G N}{3}}
$$

and the field strength of the gauge field is given in the conformal gauge by

$$
F_{+-}=B \mathrm{e}^{2 \phi_{0}+2 \rho_{0}}, \quad B^{2}=\frac{\lambda^{2} \mathrm{e}^{-4 \phi_{0}}\left(\mathrm{e}^{-2 \phi_{0}}-\frac{4 G N}{3}\right)}{\mathrm{e}^{-2 \phi_{0}}-\frac{2 G N}{3}}, \quad \mathrm{e}^{2 \rho_{0}}=\frac{2 C}{R_{0}} \cdot \frac{1}{\cosh ^{2}(r \sqrt{C})} .
$$

As in the case of the Nariai black hole, we can investigate the (in)stability of the above solution by using the eigenvalue $A$ of the Laplacian in the hyperboloid and find

$$
\frac{A}{C} \sim \frac{1}{2} \pm \frac{1}{8} g^{\frac{1}{2}}+\mathcal{O}(g), \quad g \equiv 8 G N \mathrm{e}^{2 \phi_{0}} .
$$

In the classical limit $g=0$, there does not occur any kind of the radiation in the solution. Near the classical limit $g \sim 0$, there are solutions corresponding to both of stable and instable ones. It might be surprising that there is an instable mode since the extremal solution is usually believed to be stable.

Hence, we presented the evidence [57, 114, 131] that anti-evaporation may be natural phenomenon for different types of multiple horizon BHs. It may occur also for BTZ BH 133 as it was shown in ref. [57. Note once more that multiple horizon BHs are quite exotic objects. Even if they could present in our Universe [128, 129] they should be rather rare in the present epoch. Nevertheless, even so there are indications 115, 129] that instabilities in such BHs( proliferation of de Sitter space) may become extremely important in the early Universe. In fact, their instability ( in particular, anti-evaporation) could lead to kind of topological phase transition which may represent the mechanism of inflationary Universe creation!

\section{Quantum cosmology}

In the present chapter we discuss the applications of the effective action found in chapter 3 to dilatonic cosmology. We use two forms of effective action: s-wave effective action (obtained as $2 \mathrm{~d}$ anomaly induced action after spherical reduction) and $4 \mathrm{~d}$ anomaly induced effective action. It is known that these two approximations are different 134, 135], generally speaking. They lead to qualitatively the same results only for sufficiently smooth backgrounds like black holes or not very quickly oscillating cosmologies. We mainly use $4 \mathrm{~d}$ anomaly induced effective action except first section where s-wave approximation is taken. As one can see from results of this chapter it is possible to create quantum inflationary Universe (or more generally, non-singular Universes) even in the presence of time-dependent dilaton. 


\section{1 (Non)-singular Kantowski-Sachs Universe}

It is quite common belief that two-dimensional dilatonic gravity may be useful only as toy model for the study of realistic $4 \mathrm{~d}$ gravity, especially in quantum regime. However, it is quite well-known (for example, see refs. 136, 137]) that spherical reduction of Einstein gravity leads to some specific dilatonic gravity. On the same time, spherical reduction of minimal $4 \mathrm{~d}$ matter leads to $2 \mathrm{~d}$ dilaton coupled matter.

The conformal anomaly for $2 \mathrm{~d}$ conformally invariant, dilaton coupled matter and the correspondent anomaly induced effective action have been extensively discussed in chapter 3. Using such anomaly induced effective action (i.e. working in $s$-wave and large $N$ approximation) and adding it to reduced Einstein action one may study FRW or four-dimensional Kantowski-Sachs (KS) quantum cosmology [138] in consistent way as it was done in refs. 177, 139 (for a discussion of 2d dilatonic quantum cosmology, see for example 140, 141, 142, 143, 77] and references therein).

In the (mainly numerical) study of refs. 77 it was found that most of KS cosmologies under investigation are singular at the initial stage of the Universe evolution. The interesting question is : can we construct (non) singular KS quantum cosmologies using purely analytical methods?

In the first part of this section, we answer to this question following to study of ref. [139]. Using the analogy between KS cosmology and Schwarzschild BH (or its generalizations) after the interchange of time and radial coordinates (see [138, 144]) we present the particular solution of quantum equations of motion . This solution represents non-singular KS cosmology (expanding Universe with always non-zero radius) which appears from Schwarzschild-de Sitter (or -anti de Sitter) BH after interchange of time and radial coordinates. For purely induced gravity (when cosmology is defined completely by quantum effects of matter) we present general analytical solution of quantum equations of motion. Unfortunately, in situation under discussion all given examples of KS quantum cosmologies are singular.

We start from the action of Einstein gravity with $N$ minimal real scalars and $M$ Majorana fermions. In order to apply large $N$ approach $N$ and $M$ are considered to be large, $N, M \gg 1, G$. We now assume the spherically symmetric spacetime as in (160). Working in large $N$ and $s$-wave approximation one can calculate the quantum correction to $S_{\text {red }}$ (effective action). Using $2 \mathrm{~d}$ conformal anomaly for dilaton coupled scalar and dilaton coupled spinor, one can find the anomaly induced effective action. In the case of dilaton absence such induced effective action gives the complete effective action which is valid for an arbitrary two-dimensional background. In the presence of dilaton as above the complete effective action consists of two pieces. First one is induced effective action which is given actually for any background but with accuracy up to conformally invariant functional. The second piece of it, i.e. conformally invariant functional can not be found in closed form. We use Schwinger-De Witt technique (curvature expansion) to calculate it. We keep only leading part of such expansion, for more detail and explanation, see refs. 57, 59.

We choose the conformal gauge (145), $\left(x^{ \pm} \equiv t \pm r\right)$ but it is often convenient to use the cosmological time $\tau$ instead of $t$, where the metric is given by

$$
d s^{2}=-d \tau^{2}+\mathrm{e}^{2 \rho} d r^{2}+\mathrm{e}^{-2 \phi} d \Omega^{2} .
$$

Since we have $d \tau=\mathrm{e}^{\rho} d t$, we obtain $\partial_{t}=\mathrm{e}^{\rho} \partial_{\tau}$ and $\partial_{t}^{2}=\mathrm{e}^{2 \rho}\left(\partial_{\tau}^{2}+\partial_{\tau} \rho \partial_{\tau}\right)$. 
We now consider a special solution corresponding to the (Wick-rotated) Nariai solution [126], where $\phi$ is a constant : $\phi=\phi_{0}$. Then from the effective equations written explicitly in [139], we find the solution

$$
\mathrm{e}^{-2 \phi_{0}}=\frac{(2 N+M) G}{6}+\frac{1}{2 \Lambda} \pm \frac{1}{2} \sqrt{\frac{(2 N+M)^{2} G^{2}}{9}+\frac{1}{\Lambda^{2}}-\frac{(8 N+6 M) G}{3 \Lambda}}
$$

The sign \pm in (223) should be + if we require the solution coincides with the classical one $\mathrm{e}^{-2 \phi_{0}}=\frac{1}{\Lambda}$ in the classical limit of $N, M \rightarrow 0$. On the other hand, in the solution with the - sign, we have $\mathrm{e}^{-2 \phi_{0}} \sim \frac{(3 N+2 M) G}{3} \rightarrow 0$ in the classical limit. Therefore the second solution does not correspond to any classical solution but the solution is generated by the quantum effects. The solution for $\mathrm{e}^{2 \rho}$ is given by

$$
\mathrm{e}^{2 \rho}=\left\{\begin{array}{ll}
\frac{2 C}{R_{0}} \frac{1}{\cos ^{2}(t \sqrt{C})} & \text { when } R_{0}>0 \\
-\frac{2 C}{R_{0}} \frac{1}{\cosh ^{2}(t \sqrt{C})} & \text { when } R_{0}<0
\end{array} .\right.
$$

Here $C>0$ is a constant of the integration and $R_{0}$ is $2 \mathrm{~d}$ scalar curvature, which is given by

$$
\begin{aligned}
R_{0}=2 \mathrm{e}^{-2 \rho} \partial_{t}^{2} \rho= & -\frac{3 \Lambda}{(N+M) G}\left(\frac{(2 N+M) G}{3}-\frac{1}{\Lambda}\right. \\
& \left. \pm \sqrt{\frac{(2 N+M)^{2} G^{2}}{9}+\frac{1}{\Lambda^{2}}-\frac{(8 N+6 M) G}{3 \Lambda}}\right) .
\end{aligned}
$$

Note that $4 \mathrm{~d}$ curvature $R_{4}=R_{0}+2 \mathrm{e}^{2 \phi_{0}}$ becomes a constant. It should be also noted that the solution exists for the both cases: of positive $\Lambda$ and negative $\Lambda$. In Eq.(225), the + sign corresponds to the classical limit $(N, M \rightarrow 0)$. In the limit, we obtain $R_{0} \rightarrow 2 \Lambda\left(R_{4} \rightarrow 4 \Lambda\right)$. On the other hand, the - sign in Eq. (225) corresponds to the solution with - sign in (223) generated by the quantum effect. In the classical limit for the solution, the curvature $R_{0}$ in (225) diverges as $R_{0} \sim \frac{3}{2(N+M) G} \rightarrow+\infty$. Therefore from (224), we find that $\mathrm{e}^{2 \rho}$ vanish. (Note that $R_{0}>0$ in the limit) $: \mathrm{e}^{2 \rho}=\frac{4(N+M) G C}{3 \cos ^{2}(t \sqrt{C})} \rightarrow 0$. Therefore by using (160), we obtain the following metric near the classical limit:

$$
d s^{2}=\frac{4(N+M) G C}{3 \cos ^{2}(t \sqrt{C})}\left(-d t^{2}+d r^{2}\right)+\frac{(3 N+2 M) G}{3} d \Omega^{2} .
$$

This is non-singular metric for fixed $N, M$.

It should be interesting to consider the limit $\Lambda \rightarrow 0$, where there is no de Sitter or anti-de Sitter solution at the classical level. In the limit, we can have a finite solution:

$$
\mathrm{e}^{-2 \phi_{0}} \rightarrow \frac{(3 N+2 M) G}{3}, \mathrm{e}^{2 \rho} \rightarrow \frac{(N+M) G C}{3 \cos ^{2}(t \sqrt{C})}\left(R_{0} \rightarrow \frac{6}{(N+M) G}\right)
$$

This tells that the Nariai space can be generated by the quantum effects even if $\Lambda=0$.

The obtained solution (224) (and (226)) might appear to have a singularity when $\cos ^{2}(t \sqrt{C})=0$ (for $R_{0}>0$ case) but the singularity is apparent one. In fact the scalar curvature $R_{0}$ in (225) is always constant. If we change the conformal time coordinate $t$ by the cosmological time $\tau$ in (222), we find that the time $\cos ^{2}(t \sqrt{C})=0$ corresponds to infinite future or past. In the following, we assume $R_{0}>0$ for simplicity. $R_{0}<0$ case can be easily obtained by changing the constant $C \rightarrow-C$ and analytically continuing solutions. We now change the time-coordinate by $\tau=\sqrt{\frac{2}{R_{0}}} \ln \left(\frac{1+\tan \left(t \frac{\sqrt{C}}{2}\right)}{1-\tan \left(t \frac{\sqrt{C}}{2}\right)}\right)$. Then the time 
$\cos ^{2}(t \sqrt{C})=0\left(t \sqrt{C}= \pm \frac{\pi}{2}\right)$ corresponds to $\tau= \pm \infty$. Using the cosmological time $\tau$, we obtain the following metric

$$
d s^{2}=-d \tau^{2}+\frac{2 C}{R_{0}} \cosh ^{2}\left(\tau \sqrt{\frac{R_{0}}{2}}\right) d r^{2}+\mathrm{e}^{-2 \phi_{0}} d \Omega^{2} .
$$

Here $\mathrm{e}^{-2 \phi_{0}}$ is given in (223). If we assume $r$ has the periodicity of $2 \pi$, the metric describes non-singular Kantowski-Sachs Universe, whose topology is $S_{1} \times S_{2}$. The radius of the $S_{2}$ is constant but the radius of $S_{1}$ has a minimum when $\tau=0$ and increases exponentially with the absolute value of $\tau$.

Hence we found non-singular KS cosmology which exists on classical level and which also exists on quantum level (as quantum corrected KS cosmology). This metric may be considered as the one obtained from Schwarzschild-de Sitter (Nariai) BH (for positive cosmological constant) 126] and from Schwarzschild-anti-de Sitter BH (for negative cosmological constant). To make the correspondence one has to interchange time and radial coordinates assuming corresponding Wick-rotation. It is very interesting that the last case (of negative cosmological constant) may be relevant to AdS/CFT correspondence (see next chapter). We also found non-singular KS Universe which does not have the classical limit and which is completely induced by quantum effects (even in the case of zero cosmological constant). Hence we obtained expanding Universe with the radius which is never zero. This cosmology may be interesting in frames of inflationary Universe as it can describe some sub-stage of inflationary Universe where there is effective expansion only along one (or two) space coordinates.

Let us discuss now the situation when we live in the regime where quantum (non-local) anomaly induced effective action gives major contribution to equations of motion. In other words, quantum cosmology is defined completely by quantum effects (effective gravity theory which at some point makes transition to classical gravity). As we will see in this case the equations of motion admit the analytical solutions which lead to singular KS cosmology. We consider purely induced gravity, i.e. $N, M \rightarrow \infty$ case. Then the Einstein action can be dropped away. For this case, the field equations admit the following integral of motion 139 .

$$
I_{1}=\mathrm{e}^{\rho}\left[\left(N+\frac{2}{3} M\right) \rho^{\prime}+2 N(\rho+a) \phi^{\prime}\right]
$$

Here $^{\prime}=\frac{d}{d \tau}$. For the case $\phi=$ const $=\phi_{0}$, we have the following solution of equations

$$
r(\tau)=\text { const }=\mathrm{e}^{-\phi_{0}}, \quad f(\tau)=\mathrm{e}^{\rho}=\left(f_{0}^{\prime} \tau+f_{0}\right) .
$$

For the solution (230), the scalar curvature is constant : $R=2 \mathrm{e}^{2 \phi_{0}}$. For the case $\rho=$ const $=\rho_{0}$, we have the solution of equations

$$
\begin{gathered}
\rho_{0}=-a, \quad r(\tau)=\mathrm{e}^{-\phi}=\left(c_{1} \tau+c_{2}\right)^{1+\frac{2 M}{3 N}}, \quad c_{1}, c_{2}=\text { const. } \\
c_{2}=\exp \left(\frac{-3 N \phi_{0}}{2 M+3 N}\right), \quad c_{1}=c_{2} \frac{-3 N \phi_{0}^{\prime}}{(2 M+3 N)}
\end{gathered}
$$

Here $\phi_{0}$ and $\phi_{0}^{\prime}$ are the values of $\phi$ and $\phi^{\prime}$ at $\tau=0$, respectively. For the solution (231), the scalar curvature has a singularity at $\tau=-\frac{c_{2}}{c_{1}}$. If $\rho \neq$ const, then we obtain the following special solution

$$
f(\tau)=\mathrm{e}^{\rho}=f_{0}^{\prime} \tau+f_{0}, \quad r(\tau)=\mathrm{e}^{-\phi}=c_{3}\left[a+\ln \left(f_{0}^{\prime} \tau+f_{0}\right)\right]^{1+\frac{2 M}{3 N}} .
$$


Here $f_{0}$ and $f_{0}^{\prime}$ are the values of $f=\mathrm{e}^{\rho}$ and $f^{\prime}$ at $\tau=0$ respectively. For the solution (233), the scalar curvature has a singularity when $\tau=-\frac{f_{0}}{f_{0}^{\prime}}-\frac{\mathrm{e}^{-a}}{f_{0}}$. Similarly, other cases giving singular solutions may be discussed.

Generalizing, we should note that the equation of motion admit one more integral besides $I_{1}$ in (229). It may be presented as follows

$$
0=\frac{1}{2}\left(\rho^{\prime}\right)^{2}+V(\rho), \quad V(\rho)=-\frac{6 I_{2}}{N+M} \frac{\rho+a-\alpha}{\rho+a-\beta} \mathrm{e}^{-2 \rho} .
$$

Here $\alpha \equiv-\frac{I_{1}^{2}}{8 N I_{2}}$ and $\beta \equiv-\frac{3\left(N+\frac{2}{3} M\right)^{2}}{2 N(N+M)}$. Note that $\beta$ is negative and $\alpha$ is positive (negative) when $I_{2}$ is negative (positive).

Eq. 234 tells that there would be a curvature singularity when $\rho+a \rightarrow \beta \pm 0$. In fact, when $\rho+a \sim \beta \pm 0$, we obtain from (234) $\left(\rho^{\prime}\right)^{2} \sim \frac{A}{\rho+a-\beta},\left(A \equiv \frac{12 I_{2}(\beta-\alpha) \mathrm{e}^{2(a-\beta)}}{N+M}\right.$. Therefore we find $\rho+a=$ $\beta+\left(\frac{3 A}{2}\left(\tau-\tau_{\beta}\right)\right)^{\frac{2}{3}}$. Here $\tau_{\beta}$ is a constant of the integration and $\rho+a=\beta$ when $\tau=\tau_{\beta}$. Then the scalar curvature $R$ is always singular when $\rho+a \sim \beta \pm 0$ except $\alpha=\beta$ case, when $A$ vanishes (we should note that $A$ is finite when $I_{2}=0$ ).

In the case $\alpha=\beta$, 234) can be explicitly solved to give

$$
\mathrm{e}^{\rho}= \pm \frac{12 I_{2}}{N+M}\left(\tau-\tau_{0}\right)
$$

Here $\tau_{0}$ is a constant of the integration. (235) tells that there is a singularity when $\tau=\tau_{0}$. In case of the expanding universe $(+\operatorname{sign}$ in (235) $), \phi^{\prime}=0$, i.e., $\phi$ is a constant as in the Nariai space 126] (note that there is a singularity even in this case, which is different from the Nariai space). On the other hand, in case of the shrinking universe ( - sign in (235)), there is a curvature singularity when $\tau-\tau_{0}=-\frac{N+M}{12 I_{2}} \mathrm{e}^{-a}$ besides $\tau=\tau_{0}$. The analysis of all other sub-cases for explicit analytical solutions may be presented without problems.

As it follows from above analysis in purely induced gravity case when expanding Universe is created by the matter quantum effects one always gets the curvature singularity like in the case discussed in ref. 77 . Nevertheless, it is remarkable that equations of motion in this case admit analytical solutions. Hence, using $s$-wave and large $N$ approximation we studied gravitational equations of motion with quantum corrections. The analytical solutions representing (non) singular KS cosmology are found.

In [77, it has been considered $N=12$ d dilatonic supergravity (SG), 2d dilatonic SG obtained by dimensional reduction from $N=14 \mathrm{~d}$ SG, $N=22$ d dilatonic SG and string-inspired 4d dilatonic SG. We use the conformal anomaly induced effective action due to dilaton-coupled conformal matter as a quantum correction (for $4 \mathrm{~d}$ models $s$-waves approximation is additionally applied). The solutions of the equations of motion have been numerically investigated for $2 \mathrm{~d}$ and $4 \mathrm{~d}$ FRW or 4d Kantowski-Sachs Universes with a time-dependent dilaton. The evolution of the corresponding quantum cosmological models is given for different choices of initial conditions and theory parameters. In most cases one finds quantum singular Universes. Nevertheless, there are examples of Universe non-singular at early times.

The general $N=1$ action of 2 d dilatonic supergravity is given in terms of general functions of the dilaton $C(\phi), Z(\phi), f(\phi)$ and $V(\phi)$ in section 3.6. As we are going to work in large $N$ approximation we may take into account only matter quantum effects. Since we presently considering the cosmological 
problem, we assume that all the fields depend only on time $t$. Choosing the (super)conformal gauge (145), equations of motion can be solved numerically for several parameters with the initial condition $\phi=\rho=\frac{d \phi}{d t}=\frac{d \rho}{d t}=0$ at $t=0$. Especially for the supersymmetric extension of the CGHS type model where

$$
C(\phi)=2 \mathrm{e}^{-2 \phi}, \quad Z(\phi)=4 \mathrm{e}^{-2 \phi}, \quad V(\phi)=4 \lambda \mathrm{e}^{-2 \phi} .
$$

Typical graphs are given in [77 for CGHS type model. We also calculated the two-dimensional scalar curvature $R$. (As again solution under discussion may be understood as 4d KS Universe or 2d FRW-type cosmology with non-trivial dilaton.) In Figures in [77, there appeared several types of the behavior of the conformal factor $\rho$ and dilaton field $\phi$. In a solution, both the conformal factor $\rho$ and dilaton $\phi$ increase monotonically with time and there is a curvature singularity in a finite conformal time. We should note that there was not found a solution where $\rho$ monotonically increases with time in the bosonic model. If $\rho$ diverges near the singularity, the singularity will appear in the infinite future of the cosmological time defined by

$$
d \hat{t}=\mathrm{e}^{\rho} d t
$$

Note $g_{t t}=1$ when we use the cosmological time $\hat{t}$. It should be also noted that there will not appear any singularities in the two-dimensional scalar curvature in these solutions. As a rule, $\phi$ increases monotonically in time but $\rho$ increases first and decreases later, which means that the universe expands first and then later shrinks. In another solution, $\phi$ oscillates and $\rho$ decreases with small oscillation which means that an oscillating shrinking universe is realized. The scalar oscillating curvature goes to zero.

For the model reduced from $4 \mathrm{~d}$ Einstein gravity with a cosmological term and matter described by $N$ minimal scalars coupled to the metric with spherical symmetry, we concentrate on the spherically symmetric metrics of Kantowski-Sachs form where $g_{\mu \nu}=a^{2}(t) \eta_{\mu \nu}$. Such a metric describes a Universe with a $S^{1} \times S^{2}$ spatial geometry. Typical graphs for the numerical calculation are also given in [77]. The curvature singularity seems always to appear, at least in cases under discussion. As a rule, both the dilaton field $\phi$ and the conformal factor $\rho$ increase monotonically in time as in a solution in CGHS type model. If we regard the solution as describing a universe of the Kantowski-Sachs form with the topology $S^{2} \times S^{1}$, the radius of $S^{2}$, which is given by $\mathrm{e}^{-\phi}$, decreases to zero but the radius of $S^{1}$, which is given by $\mathrm{e}^{2 \rho}$, increases. Note that the radius of $S^{1}$ corresponds to the radius of the universe when we regard this model as a two dimensional one. The four-dimensional scalar curvature increases first and goes to minus infinity. In another solution, $\phi$ increases monotonically and $\rho$ decreases at first but increases infinitely after that. This means $S^{2}$ shrinks but the $S^{1}$ factor shrinks at first and later expands. The four-dimensional scalar curvature goes to zero at first but increases to infinity later. The dilaton field $\phi$ always increases and runs away to the singularity, which means $S_{2}$ in Kantowski-Sachs universe shrinks to a point, which causes the curvature singularity. When we regard the model as a two-dimensional one, the radius of the universe goes to infinity at the final stage in the cases under discussion. We see that at $t=0$ there is no singularity in most of our cases. However, at late times there is as a rule singularity. Note that the behavior of our cosmologies at late times is not so important as other effects should define late time structure of the Universe. 
The low-energy effective action of the dilaton gravity sector in the superstring may be given by

$$
S=\int d^{4} x \sqrt{-g} \mathrm{e}^{-\phi-\phi^{*}}\left(R-6 \nabla_{\mu} \phi \nabla^{\mu} \phi^{*}\right)+\cdots .
$$

Here $\cdots$ denotes the terms containing moduli, gauge fields, which depend on the detail of the compactification, and fermionic fields. On the background where the dilaton field $\phi$ is real, one takes the non-local effective action $W$. We now assume $\phi$ is real and that the conformally flat fiducial metric is $g_{\mu \nu}=\mathrm{e}^{2 \sigma} \eta_{\mu \nu}$. Some examples of the numerical solutions of effective equations are given in [77. An interesting thing is that $4 \mathrm{~d}$ scalar curvature oscillates as a trigonometric function. In a solution, both of the dilaton field $\phi$ and the conformal factor $\sigma$ slowly increase monotonically but the scalar curvature vibrates rather quickly like trigonometric functions but the amplitude slowly increases.

Hence, unlike the case of pure Einstein gravity with conformal matter, it is more difficult to realize the inflationary Universe in quantum theory with dilaton under consideration. Nevertheless, there are cases presented above where it is possible.

\subsection{Inflationary Brans-Dicke quantum Universe}

In the present section we apply the anomaly induced effective action for dilaton coupled spinor (Section 3.4) in the construction of inflationary Brans-Dicke Universe 145.

As it has been already mentioned, Brans-Dicke theory (for a review, see [146]) represents one of the simplest examples of scalar-tensor (or dilatonic) gravities where the background is described by the metric and the dilaton. Even low-energy string effective action (for a recent review, see 147) may be considered as some kind of BD theory (with higher order terms).

Let us start from the standard Brans-Dicke 4-dimensional action in the Jordan frame:

$$
S_{B D}=\frac{1}{16 \pi} \int d^{4} x \sqrt{-g}\left[\phi R-\frac{\omega}{\phi}\left(\nabla_{\mu} \phi\right)\left(\nabla^{\mu} \phi\right)\right]+S_{M},
$$

where $\phi$ is the Brans-Dicke (dilaton) field with $\omega$ being the coupling constant and $S_{M}$ is the matter action.

It has been argued (for a review, see [148]) that only the action in the Einstein frame is physically relevant. For this reason, one may prefer working within this frame, performing the following transformations

$$
\tilde{g}_{\mu \nu}=G \phi g_{\mu \nu}, \quad \tilde{\phi}=\sqrt{2 \omega+3 /(16 \pi)} \ln \phi \quad 2 \omega+3>0 .
$$

Thus, the classical action in the Einstein frame reads

$$
S=\int d^{4} x \sqrt{-\tilde{g}(x)}\left[\frac{\tilde{R}}{16 \pi G}-\frac{1}{2}\left(\tilde{\nabla}_{\mu} \tilde{\phi}\right)\left(\tilde{\nabla}^{\mu} \tilde{\phi}\right)+\exp (A \tilde{\phi}) L_{M}(\tilde{g})\right],
$$

where $A=-8 \sqrt{\frac{\pi G}{2 \omega+3}}$. Below, we consider the theory in the Einstein frame as physical theory, (no tilde will be written). As matter Lagrangian we take the one associated with $N$ massless (Dirac) spinors, i.e.

$$
L_{M}=\sum_{i=1}^{N} \bar{\psi}_{i} \gamma^{\mu} \nabla_{\mu} \psi^{i}
$$


The choice (242) is made only for the sake of simplicity.

Our purpose here will be to show the role of quantum effects associated with dilaton coupled spinors to cosmological problems in BD gravity. Assuming $N$ sufficiently large (to allow for the large $N$ approximation) one can neglect in such investigation the proper quantum gravity corrections. The corresponding 4d anomaly induced EA for dilaton coupled spinors (section 3.4) will be used. Hence, starting from the theory with the action

$$
S=\int d^{4} x \sqrt{-g}\left[\frac{R}{16 \pi G}-\frac{1}{2}\left(\nabla_{\mu} \phi\right)\left(\nabla^{\mu} \phi\right)+\exp (A \phi) \sum_{i=1}^{N} \bar{\psi}_{i} \gamma^{\mu} \nabla_{\mu} \psi^{i}\right],
$$

we will discuss FRW type cosmologies

$$
d s^{2}=-d t^{2}+a(t)^{2} d l^{2}
$$

where $d l^{2}$ is the line metric element of a 3 -dimensional space with constant curvature $\Sigma$, namely $k=1$ $\left(\Sigma=S^{3}\right), k=0\left(\Sigma=R^{3}\right)$ or $k=-1\left(\Sigma=H^{3}\right)$. Introducing the conformal time $\eta$, one gets a space-time which is conformally related to an ultrastatic space-time with constant curvature spatial section $\bar{M}$, namely

$$
g_{\mu \nu}=e^{2 \sigma(\eta)} \bar{g}_{\mu \nu},
$$

The computation of the anomaly induced EA for the dilaton coupled spinor field has been done in Section 3.4:

$$
\begin{aligned}
W=\int & d^{4} x \sqrt{-\bar{g}}\left\{b \bar{F} \sigma_{1}+2 b^{\prime} \sigma_{1}\left[\square^{2}+2 \bar{R}^{\mu \nu} \bar{\nabla}_{\mu} \bar{\nabla}_{\nu}-\frac{2}{3} \bar{R} \square+\frac{1}{3}\left(\bar{\nabla}^{\mu} \bar{R}\right) \bar{\nabla} \mu\right] \sigma_{1}\right. \\
& \left.+b^{\prime} \sigma_{1}\left(\bar{G}-\frac{2}{3} \square \bar{R}\right)-\frac{1}{18}\left(b+b^{\prime}\right)\left[\bar{R}-6 \square \sigma_{1}-6\left(\bar{\nabla}_{\mu} \sigma_{1}\right)\left(\bar{\nabla}^{\mu} \sigma_{1}\right)\right]^{2}\right\},
\end{aligned}
$$

where $\sigma_{1}=\sigma+A \phi / 3$. For Dirac spinors $b=\frac{3 N}{60(4 \pi)^{2}}, b^{\prime}=-\frac{11 N}{360(4 \pi)^{2}}$.

Generally speaking, it should be noted that the complete one-loop EA is given by the anomalyinduced EA presented above, plus some conformally invariant functional which is the EA computed in the reference metric $\bar{g}_{\mu \nu}$. In our case, this second term is rather trivial (actually, it is a $k$-dependent constant), being the EA of a free spinor field in an ultrastatic space-time with constant spatial section (for a discussion of such effective actions, see [149]). For example, for the flat case $(k=0)$, which we only consider, $\bar{g}_{\mu \nu}=\eta_{\mu \nu}$, and as a consequence, $W$ gives the complete non-trivial EA! Then, the effective action becomes

$$
W=V_{3} \int d \eta\left\{2 b^{\prime} \sigma_{1} \sigma_{1}^{\prime \prime \prime \prime}-2\left(b+b^{\prime}\right)\left(\sigma_{1}^{\prime \prime}-\sigma_{1}^{\prime 2}\right)^{2}\right\} .
$$

Here, $V_{3}$ is the (infinite) volume of 3-dimensional flat space and ${ }^{\prime} \equiv d / d \eta$ and $\sigma=\ln a$ where $a(\eta)$ is the scale factor.

The total one-loop EA may be written adding to $W$ the classical action for $k=0$ :

$$
S=V_{3} \int d \eta\left[\frac{6}{16 \pi G}\left(\sigma^{\prime \prime}+{\sigma^{\prime}}^{2}\right) \mathrm{e}^{2 \sigma}+\frac{1}{2}{\phi^{\prime}}^{2} \mathrm{e}^{2 \sigma}\right] .
$$

Having in mind the inflationary Universe solution, one can get the special solution of effective equations as

$$
a(\eta)=\frac{1}{H \eta}, \quad \phi^{\prime}(\eta)=\frac{1}{H_{1} \eta},
$$


where $H$ and $H_{1}$ are some constants. Their explicit values are:

$$
H_{1}=\sqrt{4 \pi G}\left[-\frac{3}{16} \sqrt{2 \omega+3} \pm \sqrt{\frac{81}{4}(2 \omega+3)-\frac{1}{6}}\right], \quad H^{2} \simeq-\frac{1}{16 \pi G b^{\prime}}-\frac{1}{24 H_{1}^{2} b^{\prime}}
$$

Transforming back to the physical time $t$, one obtains the $a(t)=1 / C \exp (\mp H t)$. Choosing the plus sign gives rise to the inflationary universe (in dilaton absence such solution has been found in refs. [150, 151]). In this case, $\phi \sim \frac{H}{H_{1}} t$, namely the dilaton is expanding with time, but much slower than the scale factor.

Summarizing, we have presented explicitly one special analytic solution describing Brans-Dicke nonsingular Universe with a (much slower) expanding dilaton. It should be noted that this is a purely quantum solution which does not exist at classical level. In similar way one can study Brans-Dicke quantum cosmologies for other types of dilaton coupled matter, as well as their properties (like stability) at late times. It is interesting that induced dilatonic gravities may be studied in a similar fashion. For example, starting from the Lagrangian of local superconformally invariant $\mathcal{N}=4$ super YM theory in the background of $\mathcal{N}=4$ conformal supergravity (see [152, 153, 154] for introduction and review) one can find Weyl anomaly 155 with account of dilaton for such YM theory. Then, the construction of quantum inflationary Universe from super YM theory is also possible.

\subsection{Primordial wormholes at the early Universe}

The wormholes are puzzling topological objects (kind of handles of topological origin) which attract much attention in General Relativity for years. It is not strange as they may be considered as bridges joining two different Universes or two separate regions of the same Universe (for an introduction, see refs. [156, 157]). There are many speculations related with hypothetical effects which may be expected near wormholes.

Moreover, it is known that wormholes usually cannot occur as classical solutions of gravity (with matter) due to violations of energy conditions [156, 157]. Nevertheless, one can expect that primordial wormholes may present at the very early Universe where quantum effects play an essential role (or yet in fundamental M-theory, see,for example, [158]).

Indeed as it was shown in ref. 159] (see also [160]) using quantum stress tensor for conformal scalar on spherically symmetric space and in ref. 161] using one-loop effective action in large $N$ and $s$-wave approximation for minimal scalar there may exist semiclassical quantum solution corresponding to a Lorentzian wormhole connecting two asymptotically flat regions of the Universe. The corresponding spherically symmetric wormhole solution has been found numerically in both approximations 159, 161 as well as analytically 161. That shows the principal possibility of inducing primordial wormholes at the early Universe (in its quantum regime). However above discussion has been limited strictly to scalar matter. And what happens if other types of matter are included? Hence, the very natural question is: Can primordial wormholes be induced from GUTs at the early Universe?

In this section, following to ref. 162] we give positive answer to this question for spherically symmetric wormholes. As we will see the object under consideration may be understood as $4 \mathrm{~d}$ wormhole or as $2 \mathrm{~d}$ topological object with non-trivial dilaton. We use $4 \mathrm{~d}$ conformal anomaly induced effective action 
in one-loop and large $N$ approximation. Then the effective equations for an arbitrary massless GUT containing conformal scalars, spinors and vectors may be explicitly obtained for two forms of spherically symmetric background. Numerical wormhole solution of these equations may be obtained for $\mathcal{N}=4$ super Yang-Mills theory. It is observed that it depends on the choice of the initial conditions.

Let us start from Einstein gravity with $N_{0}$ conformal scalars $\chi_{i}, N_{1}$ vectors $A_{\mu}$ and $N_{1 / 2}$ Dirac spinors $\psi_{i}$. The above matter content is typical for asymptotically free GUT at high energies as interaction terms and masses at strong curvature are negligible due to asymptotic freedom. For finite GUTs or asymptotically non -free GUTs one should not include masses if consider only conformally invariant theories and interaction plays no role as anyway we consider purely gravitational background.

The convenient choice for the spherically symmetric space-time is the following:

$$
d s^{2}=f(\phi)\left[f^{-1}(\phi) g_{\mu \nu} d x^{\mu} d x^{\nu}+r_{0}^{2} d \Omega\right] .
$$

where $\mu, \nu=0,1, g_{\mu \nu}$ and $f(\phi)$ depend only from $x^{1}$ and $r_{0}^{2}$ is non-essential constant.

The calculation of matter effective action on the background (251) may be done in the standard way as it was presented in chapter 3. In the calculation of effective action, one presents effective action as $: \Gamma=\Gamma_{i n d}+\Gamma\left[1, g_{\mu \nu}^{(4)}\right]$ where $\Gamma_{\text {ind }}=\Gamma\left[f, g_{\mu \nu}^{(4)}\right]-\Gamma\left[1, g_{\mu \nu}^{(4)}\right]$ is conformal anomaly induced action, $g_{\mu \nu}^{(4)}$ is metric (251) without multiplier in front of it, i.e., $g_{\mu \nu}^{(4)}$ corresponds to

$$
d s^{2}=\left[\tilde{g}_{\mu \nu} d x^{\mu} d x^{\nu}+r_{0}^{2} d \Omega\right], \quad \tilde{g}_{\mu \nu} \equiv f^{-1}(\phi) g_{\mu \nu} .
$$

The conformal anomaly for above matter is written in section 3.3 (no dilaton)

$$
T=b\left(F+\frac{2}{3} \square R\right)+b^{\prime} G+b^{\prime \prime} \square R
$$

where $b=\frac{\left(N_{0}+6 N_{1 / 2}+12 N_{1}\right)}{120(4 \pi)^{2}}, b^{\prime}=-\frac{\left(N_{0}+11 N_{1 / 2}+62 N_{1}\right)}{360(4 \pi)^{2}}, b^{\prime \prime}=0$.

Conformal anomaly induced effective action $\Gamma_{\text {ind }}$ is:

$$
\begin{aligned}
& W=b \int d^{4} x \sqrt{-g} F \sigma+b^{\prime} \int d^{4} x \sqrt{-g}\left\{\sigma\left[2 \square^{2}+4 R^{\mu \nu} \nabla_{\mu} \nabla_{\nu}-\frac{4}{3} R \square+\frac{2}{3}\left(\nabla^{\mu} R\right) \nabla_{\mu}\right] \sigma\right. \\
& \left.+\left(G-\frac{2}{3} \square R\right) \sigma\right\}-\frac{1}{12}\left(b^{\prime \prime}+\frac{2}{3}\left(b+b^{\prime}\right)\right) \int d^{4} x \sqrt{-g}[R-6 \square \sigma-6(\nabla \sigma)(\nabla \sigma)]^{2}
\end{aligned}
$$

where $\sigma=\frac{1}{2} \ln f(\phi)$, and $\sigma$-independent terms are dropped. All 4-dimensional quantities (curvatures, covariant derivatives) in Eq.(254) should be calculated on the metric (252). $\Gamma\left[1, g_{\mu \nu}^{(4)}\right]$ is given as follows:

$$
\Gamma\left[1, g_{\mu \nu}^{(4)}\right]=\int d^{4} x \sqrt{-g}\left\{\left[b F+b^{\prime} G+\frac{2 b}{3} \square R\right] \ln \frac{R}{\mu^{2}}\right\}+\mathcal{O}\left(R^{3}\right)
$$

where $\mu$ is mass parameter, all the quantities are calculated on the background (252). The condition of application of above expansion is $|R|<R^{2}$ (curvature is nearly constant)..

One can get the equations of motion from the above effective Lagrangian $S+\Gamma$ which is effectively two-dimensional object. Note that extra scalar (dilaton) appears after reduction to two-dimensional gravity. In the following, it is convenient to work in the conformal gauge (145) after considering the variation of the effective action $\Gamma+S$ with respect to two-dimensional metric $g_{\mu \nu}$ and $\sigma$. Note that the 
tensor $g_{\mu \nu}$ under consideration is the product of the original metric tensor and the $\sigma$-function $\mathrm{e}^{-2 \sigma}$, the equations given by the variations over $g_{\mu \nu}$ are the combinations of the equations given by the variation over the original metric and $\sigma$-equation.

It often happens that we can drop the terms linear in $\sigma$. In particular, one can redefine the corresponding source term as it is in the case of infra-red sector of $4 \mathrm{~d}$ quantum gravity. In the following, we only consider this case. Then the equations of motion given by variations of $S+\Gamma_{i n d}+\Gamma\left[1, g_{\mu \nu}^{(4)}\right]$ are written in 162] ( they are too lengthy to write down it here). Now the real four-dimensional metric is given by

$$
d s^{2}=-\mathrm{e}^{2 \sigma+2 \rho} d x^{+} d x^{-}+r_{0}^{2} \mathrm{e}^{2 \sigma} d \Omega^{2} .
$$

It is convenient to make the following change: $\partial_{r}=\mathrm{e}^{\rho+\sigma} \partial_{l}$. Using the new radial coordinate $l$, the metric (256) is rewritten as follows:

$$
d s^{2}=-f(l) d t^{2}+d l^{2}+r(l)^{2} d \Omega^{2}, \quad f(l)=\mathrm{e}^{2 \sigma+2 \rho}, \quad r(l)=r_{0} \mathrm{e}^{\sigma}
$$

Here $f(l)$ is called a redshift function and $r(l)$ is a shape function. If $f(l)$ and $r(l)$ are smooth positivedefinite functions, which satisfy the conditions:

$$
\begin{aligned}
& f(l) \rightarrow 1, r(l) \rightarrow l \quad \text { when }|l| \rightarrow \infty \\
& f(l), r(l) \rightarrow \text { finite } \quad \text { when }|l| \rightarrow 0,
\end{aligned}
$$

the metric expresses the wormhole which connects two asymptotically flat universes.

In the choice of the metric (257), the equations of motion contain the 6th order derivatives of $\rho, \partial_{l}^{6} \rho$ and the 4 th order derivatives of $\sigma$. Therefore we need to impose the initial conditions including 5 th order derivative of $\rho$ and 3 rd order derivative of $\sigma$, say:

$$
\left.\partial_{l} \rho\right|_{l=0}=\left.\partial_{l}^{2} \rho\right|_{l=0}=\left.\partial_{l}^{3} \rho\right|_{l=0}=\left.\partial_{l}^{4} \rho\right|_{l=0}=\left.\partial_{l}^{5} \rho\right|_{l=0}=\left.\partial_{l} \sigma\right|_{l=0}=\left.\partial_{l}^{2} \sigma\right|_{l=0}=\left.\partial_{l}^{3} \sigma\right|_{l=0}=0 .
$$

We should also note that all the equations of motion are not the dynamical equations of motion but the combinations with the constraint. One can prove numerically that at least for some initial conditions GUTs at the early Universe may help in producing of primordial wormholes. It is of course the open question which particular initial conditions lead to more stable configuration. Moreover, due to complicated structure of field equations and initial conditions itself we cannot classify from the very beginning the initial conditions as supporting (or not) wormholes production. Nevertheless for any specific GUT under discussion above study may be easily repeated and principal possibility of wormholes inducing may be shown at least numerically.

In summary, we discussed the applications of the effective action in the construction of consistent non-singular Universe induced by quantum effects. The examples of such cosmologies even with nontrivial dilaton have been indeed presented. Note that the presence of dilaton could be consistent with observations as it quickly decays to zero. Other cosmological solutions may be constructed in the similar fashion. In order to understand their relevance to observational data one should study their properties in more detail. 


\section{Dilatonic gravity and Anti-de Sitter/Conformal Field Theory correspondence}

In the present Chapter we discuss the properties of classical five dimensional dilatonic gravity (IIB supergravity bosonic sector), in particularly, its classical solutions. Using bulk-boundary correspondence this study may help in understanding of strong coupling limit of quantum gauge field theory. Hence, volume classical physics turns out to describe simultaneously surface quantum physics. Note that there exists an excellent review on AdS/CFT correspondence [163] so we concentrate mainly on issues where the presence of dilaton is important as it changes the properties of theory drastically.

\subsection{Axion-dilatonic conformal anomaly from AdS/CFT correspondence}

In this section, we show how one can find large $N$ conformal anomaly with dilaton and axion from $5 \mathrm{~d}$ AdS dilatonic gravity. First of all, we remind few simple facts about anti-de Sitter space. $D$ dimensional antide Sitter space can be realized by imposing a constraints on $D+1$ coordinates: $-x_{0}^{2}+x_{1}^{2}+\cdots x_{D-1}^{2}-x_{D}^{2}=$ $-L^{2}$. From this realization, it is easy to see that this space has $S O(D-1,2)$ symmetry as isometry. The algebra of $S O(D-1,2)$ symmetry is the same as the algebra of conformal transformations acting on $D-1$ dimensional Minkowski space.

In an adequate coordinate choice (actually, there are many different coordinate choices), the metric on the $D$-dimensional anti-de Sitter space is given by

$$
d s_{\text {AdS }}^{2}=\rho^{-2} d \rho^{2}+\rho^{-1} \sum_{i, j=0}^{D-2} \eta_{i j} d x^{i} d x^{j} .
$$

Here $\eta$ is the metric on the flat $D-1$-dimensional Minkowski space. We should note that there is a boundary when $\rho$ vanishes. The topology of the boundary is almost that of the $D-1$ dimensional Minkowski space, or more exactly, Minkowski space with a point at infinity, that is, topologically compactified Minkowski space. On the boundary manifold, $S O(D-1,2)$ acts exactly as usual conformal transformation. When we consider the surface with fixed finite $\rho$, there is a correction proportional $\rho$ only in the conformal boost but the correction vanishes just on the boundary, that is, in the limit that $\rho$ vanishes.

AdS/CFT correspondence (or bulk/boundary) is conjectured in ref. 164. When $N$ p-branes in superstring theory or so-called M-theory coincide with each other and the coupling constant is small, the classical supergravity on $\operatorname{AdS}_{D=d+1=p+2}$, which is the low energy effective theory of superstring or Mtheory, is, in some sense, dual to large $N$ conformal field theory on $M^{d}$, which is the boundary of the AdS. For example, $d=2$ case corresponds to $(4,4)$ superconformal field theory, $d=4$ case corresponds to $U(N)$ or $S U(N) \mathcal{N}=4$ super Yang Mills theory and $d=6$ case to $(0,2)$ superconformal field theory.

The conjecture tells that partition function in $d$-dimensional conformal field theory is given in terms of the classical action in $d+1$-dimensional gravity theory:

$$
Z_{d}\left(\phi_{0}\right)=\mathrm{e}^{-S_{\text {AdS }}\left(\phi^{\text {classical }}\left(\phi_{0}\right)\right)} .
$$


Here $\phi_{0}$ is the value of the field $\phi$ on the boundary and $\phi^{\text {classical }}\left(\phi_{0}\right)$ is a field on bulk background, which is AdS, given by solving the equations of motion with the boundary value $\phi_{0}$ on $M^{d}$. $S_{\text {AdS }}\left(\phi^{\text {classical }}\left(\phi_{0}\right)\right)$ is the classical gravity action on AdS. When we substitute the classical solution into the action, the action, in general, contains infrared divergences coming from the infinite volume of AdS. Then we need to regularize the infrared divergence. It is known that as a result of the regularization and the renormalization there often appear anomalies. In ref. 165] Witten made the proposal how to calculate the conformal anomaly( quantum object) from classical gravity (bulk) side. This proposal has been worked out in detail in ref.166 (for Einstein gravity) where it was shown that the conformal (Weyl) anomaly may be recovered after regularizing the above infrared divergence (from bulk side- Einstein theory). The usual result for conformal anomaly of boundary QFT thus may be reproduced. This is a kind of IR-UV duality.

In [167, the conformal anomaly from 5d dilatonic gravity was investigated. Let us briefly describe this calculation. We start from the action of $d+1$-dimensional dilatonic gravity with boundary terms

$$
\begin{aligned}
S= & \frac{1}{16 \pi G}\left[\int_{M_{d+1}} d^{d+1} x \sqrt{-\hat{G}}\left\{\hat{R}+X(\phi)(\hat{\nabla} \phi)^{2}+Y(\phi) \hat{\Delta} \phi+4 \lambda^{2}\right\}\right. \\
& \left.+\int_{M_{d}} d^{d} x \sqrt{-\hat{g}}\left(2 \hat{\nabla}_{\mu} n^{\mu}+\alpha\right)\right] .
\end{aligned}
$$

Here $M_{d+1}$ is $d+1$ dimensional manifold, which is identified with $\operatorname{AdS}_{d+1}$ and $n_{\mu}$ is the unit vector normal to the boundary manifold $M_{d}$. $\alpha$ is a parameter which is chosen properly. The boundary terms play a role for cancellation of the leading infrared divergences which guarantee the system depends only on the boundary value. In Eq. 262) $X(\phi)$ and $Y(\phi)$ are arbitrary functions depending on dilaton $\phi$. Note that the arbitrariness of $X(\phi)$ and $Y(\phi)$ can be absorbed into the redefinition of the dilaton. In fact, if we define new dilaton field $\varphi$ by

$$
\varphi \equiv \int d \phi \sqrt{2 V(\phi)}, \quad V(\phi) \equiv X(\phi)-Y^{\prime}(\phi),
$$

the action (262) can be rewritten by using partial integration as follows:

$$
S=\frac{1}{16 \pi G}\left[\int d^{d+1} x \sqrt{-\hat{G}}\left\{\hat{R}+2(\hat{\nabla} \varphi)^{2}+4 \lambda^{2}\right\}+\int_{M_{d}} d^{d} x \sqrt{-\hat{g}}\left(2 \hat{\nabla}_{\mu} n^{\mu}+\alpha+Y(\phi) n^{\mu} \partial_{\mu} \phi\right)\right] .
$$

The $\phi$ dependent term on $M_{d}$ does not finally contribute to Weyl anomaly. We keep, however, $X(\phi)$ and $Y(\phi)$ as arbitrary functions for the later convenience. Note also that boundary term may be used to present the action as the functional of fields and their first derivatives [168].

As in 169], we choose the metric $\hat{G}_{\mu \nu}$ on $M_{d+1}$ and the metric $\hat{g}_{\mu \nu}$ on $M_{d}$ in the following form:

$$
d s^{2} \equiv \hat{G}_{\mu \nu} d x^{\mu} d x^{\nu}=\frac{l^{2}}{4} \rho^{-2} d \rho d \rho+\sum_{i=1}^{d} \hat{g}_{i j} d x^{i} d x^{j}, \quad \hat{g}_{i j}=\rho^{-1} g_{i j} .
$$

Here $l$ is related with $\lambda^{2}$ by $4 \lambda^{2}=-d(d-1) / l^{2}$. Note that the expression of the metric (265) has a redundancy. In fact, the expression (265) is invariant if we change $\rho$ and $g_{i j}$ by

$$
\delta \rho=\delta \sigma \rho, \quad \delta g_{i j}=\delta \sigma g_{i j} .
$$

Here $\delta \sigma$ is a constant parameter of the transformation. The transformation (266) can be regarded as the scale transformation on $M_{d}$. 
When $d$ is even, we can expand $\phi$ and $g_{i j}$ as power series of $\rho$ :

$$
\begin{aligned}
\phi & =\phi_{(0)}+\rho \phi_{(1)}+\rho^{2} \phi_{(2)}+\cdots \rho^{\frac{d}{2}} \phi_{(d / 2)}-\rho^{\frac{d}{2}} \ln \rho \psi+\mathcal{O}\left(\rho^{\frac{d}{2}+1}\right) \\
g_{i j} & =g_{(0) i j}+\rho g_{(1) i j}+\rho^{2} g_{(2) i j}+\cdots+\rho^{\frac{d}{2}} g_{(d / 2) i j}-\rho^{\frac{d}{2}} \ln \rho h_{i j}+\mathcal{O}\left(\rho^{\frac{d}{2}+1}\right) .
\end{aligned}
$$

Here we regard $\phi_{(0)}$ and $g_{(0) i j}$ as independent fields on $M_{d}$ and $\phi_{(l)}, g_{(l) i j}(l=1,2, \cdots), \psi$ and $h_{i j}$ as fields depending on $\phi_{(0)}$ and $g_{(0) i j}$ by using equations of motion. Then the action (264) diverges in general since the action contains the infinite volume integration on $M_{d+1}$. The action is regularized by introducing the infrared cutoff $\mathrm{f}^{5}$ and replacing

$$
\int d^{d+1} x \rightarrow \int d^{d} x \int_{\epsilon} d \rho,\left.\quad \int_{M_{d}} d^{d} x(\cdots) \rightarrow \int d^{d} x(\cdots)\right|_{\rho=\epsilon} .
$$

The terms proportional to the (inverse) power of $\epsilon$ in the regularized action are invariant under the scale transformation

$$
\delta g_{(0) \mu \nu}=2 \delta \sigma g_{(0) \mu \nu}, \quad \delta \epsilon=2 \delta \sigma \epsilon
$$

which corresponds to (266). Then the subtraction of these terms proportional to the inverse power of $\epsilon$ does not break the invariance under the scale transformation. When $d$ is even, however, the term proportional to $\ln \epsilon$ appears. The term is not invariant under the scale transformation (269) and the subtraction of the $\ln \epsilon$ term breaks the invariance. The variation of the $\ln \epsilon$ term under the scale transformation 269) is finite when $\epsilon \rightarrow 0$ and should be canceled by the variation of the finite term (which does not depend on $\epsilon$ ) in the action since the original action (264) is invariant under the scale transformation. Therefore the $\ln \epsilon$ term $S_{\ln }$ gives the Weyl anomaly $T$ of the action renormalized by the subtraction of the terms which diverge when $\epsilon \rightarrow 0$

$$
S_{\ln }=-\frac{1}{2} \int d^{4} x \sqrt{-g_{(0)}} T .
$$

First we consider the case of $d=2$. Choosing $\alpha$ to satisfy the equation $\alpha=\frac{2}{l}$ and using the replacement in (270), we find the action (264) has the following form

$$
S=-\frac{1}{16 \pi G} \frac{l}{2} \ln \epsilon \int d^{2} x \sqrt{-g_{(0)}}\left\{R_{(0)}+X\left(\phi_{(0)}\right)\left(\nabla \phi_{(0)}\right)^{2}+Y\left(\phi_{(0)}\right) \Delta \phi_{(0)}\right\}+\text { finite terms } .
$$

Then we find an expression of the Weyl anomaly $T$ by using (270)

$$
T=\frac{l}{16 \pi G}\left\{R_{(0)}+X\left(\phi_{(0)}\right)\left(\nabla \phi_{(0)}\right)^{2}+Y\left(\phi_{(0)}\right) \Delta \phi_{(0)}\right\}
$$

This result could be compared with the UV-calculation of the conformal anomaly of dilaton coupled $N$ scalars and $M$ Majorana dilaton coupled spinors from chapter 3:

$$
\begin{aligned}
& \frac{l}{16 \pi G}=\frac{2 N+M}{48 \pi}, \quad \frac{l}{16 \pi G} X\left(\phi_{(0)}\right)=-\frac{N}{4 \pi}\left(\frac{f^{\prime \prime}}{2 f}-\frac{f^{\prime 2}}{4 f^{2}}\right)-\frac{M}{24 \pi}\left(\frac{g^{\prime \prime}}{g}-\frac{g^{\prime 2}}{g^{2}}\right), \\
& \frac{l}{16 \pi G} Y\left(\phi_{(0)}\right)=-\frac{N}{4 \pi} \frac{f^{\prime}}{2 f}-\frac{M}{24 \pi} \frac{g^{\prime}}{g} .
\end{aligned}
$$

\footnotetext{
${ }^{5}$ From the viewpoint of the supergravity, the cutoff $\epsilon$ can be regarded as infrared (IR) cutoff regularizing the infinte volume of $M_{d+1}$, which can be $\mathrm{AdS}_{d+1}$. From the viewpoint of the $d$-dimensional field theory, the cutoff $\epsilon$ corresponds to the ultraviolet (UV) cutoff as in the present calculation of conformal anomaly. This is nothing but the IR/UV duality in AdS/CFT correspondence.
} 
The above result should give the conformal anomaly computed from the asymptotic symmetry algebra of $\mathrm{AdS}_{3}$ with dilaton, as it was the case in the absence of dilaton 170.

We now consider the case of four dimensions. The calculation similar to that in 2 dimensions leads to the term $S_{\text {ln }}$ proportional to $\ln \epsilon$ in the action, which contains $g_{(0) i j}, g_{(1) i j}, \phi_{(0)}$, and $\phi_{(1)}$. Using the equations of motion given by the variation of $g_{(1) i j}$ and $\phi_{(1)}, g_{(1) i j}$ and $\phi_{(1)}$ can be solved with respect to $g_{(0) i j}$ and $\phi_{(0)}$. After deleting $g_{(1) i j}$ and $\phi_{(1)}$, we obtain the following expression :

$$
\begin{aligned}
S_{\mathrm{ln}} & =\frac{l^{3}}{16 \pi G} \int d^{4} x \sqrt{-g_{(0)}}\left[\frac{1}{8} R_{(0) i j} R_{(0)}^{i j}-\frac{1}{24} R_{(0)}^{2}+\frac{1}{2} R_{(0)}^{i j} \partial_{i} \varphi_{(0)} \partial_{j} \varphi_{(0)}\right. \\
- & \left.\frac{1}{6} R_{(0)} g_{(0)}^{i j} \partial_{i} \varphi_{(0)} \partial_{j} \varphi_{(0)}+\frac{1}{4}\left\{\frac{1}{\sqrt{-g_{(0)}}} \partial_{i}\left(\sqrt{-g_{(0)}} g_{(0)}^{i j} \partial_{j} \varphi_{(0)}\right)\right\}^{2}+\frac{1}{3}\left(g_{(0)}^{i j} \partial_{i} \varphi_{(0)} \partial_{j} \varphi_{(0)}\right)^{2}\right] .
\end{aligned}
$$

Here we choose $\alpha$ to be $\alpha=\frac{6}{l}$ and using $V\left(\phi_{(0)}\right)$, which is defined in 263), the field $\varphi_{(0)}$ is defined by $\varphi_{(0)} \equiv \int d \phi_{(0)} \sqrt{2 V\left(\phi_{(0)}\right)}$ in a similar way to 263$)$.

The Weyl anomaly coming from the multiplets of $\mathcal{N}=4$ supersymmetric $U(N)$ or $S U(N)$ Yang-Mills conformally coupled with $\mathcal{N}=4$ conformal supergravity was calculated in 155 :

$$
\begin{aligned}
T= & -\frac{N^{2}}{4(4 \pi)^{2}}\left[2\left(R_{i j} R^{i j}-\frac{1}{3} R^{2}\right)+F^{i j} F_{i j}\right. \\
& \left.+4\left\{2\left(R^{i j}-\frac{1}{3} R g^{i j}\right) \partial_{i} \varphi^{*} \partial_{j} \varphi+\left|\frac{1}{\sqrt{-g}} \partial_{i}\left(\sqrt{-g} g^{i j} \partial_{j} \varphi\right)\right|^{2}\right\}+\cdots\right] .
\end{aligned}
$$

Here $F_{i j}$ is the field strength of SU(4) gauge fields, $\varphi$ is a complex scalar field which is a combination of dilaton and RR scalar (or axion) and "..." expresses the terms containing other fields in $\mathcal{N}=4$ conformal supergravity multiplet and higher powers of the fields.

If we choose

$$
\frac{l^{3}}{16 \pi G}=\frac{2 N^{2}}{(4 \pi)^{2}}
$$

and consider the background where only gravity and the real part of the scalar field $\varphi$ in the $\mathcal{N}=$ 4 conformal supergravity multiplet are non-trivial and other fields vanish in (275), Eq.(274) exactly reproduces the result in (275) by using (270). It is interesting that last term in (275) actually gives the correct dilatonic dependent contribution to conformal anomaly of dilaton coupled electromagnetic field. It corrects the failed result obtained in section 3.5 by convenient methods.

Hence, we got $d=2$ and $d=4$ holographic conformal anomaly for dilaton coupled theories from supergravity side. The results of this study give further check of AdS/CFT correspondence in the presence of dilaton. We should note that it has been also found agreement 171, 172 between AdS/CFT conformal anomaly and perturbative QFT anomaly in $\mathcal{N}=2$ SCFT even in the next to leading order term.

In [173], it was investigated $5 \mathrm{~d}$ dilaton-axionic AdS gravity, whose action is actually motivated by bosonic sector of IIB SG model due to Gibbons, Green and Perry 174 which contains axion. Such investigation is necessary in order to check the possibility that the fields besides the graviton and dilaton , say axion field, could not spoil AdS/CFT correspondence. Therefore it would be important that background axion and dilaton are kept in the calculation of conformal anomaly. The starting action in 
173 is given by

$$
\begin{array}{r}
S=\frac{1}{16 \pi G}\left\{\int _ { M _ { d + 1 } } d ^ { d + 1 } x \sqrt { - \hat { G } } \left(\hat{R}+X(\phi, \chi)(\hat{\nabla} \phi)^{2}+Y(\phi, \chi) \hat{\Delta} \phi\right.\right. \\
\left.\left.+Z(\phi, \chi)(\hat{\nabla} \chi)^{2}+W(\phi, \chi) \hat{\Delta} \chi+4 \lambda^{2}\right)+\int_{M_{d}} d^{d} x \sqrt{-\hat{g}}\left(2 \hat{\nabla}_{\mu} n^{\mu}+\alpha\right)\right\} .
\end{array}
$$

Here $\chi$ is axion (or RR-scalar). The bosonic action of IIB SG in 174 represents the special case of the above action. If we define

$$
\left(\begin{array}{l}
X^{1} \\
X^{2}
\end{array}\right) \equiv\left(\begin{array}{c}
\phi \\
\chi
\end{array}\right), \quad h_{\mu \nu} \equiv\left(\begin{array}{cc}
X-\frac{\partial Y}{\partial \phi} & -\frac{1}{2}\left(\frac{\partial W}{\partial \phi}+\frac{\partial Y}{\partial \chi}\right) \\
-\frac{1}{2}\left(\frac{\partial W}{\partial \phi}+\frac{\partial Y}{\partial \chi}\right) & Z-\frac{\partial W}{\partial \chi}
\end{array}\right)
$$

the action (277) can be rewritten, after partial integration, in the form of the sigma model, whose target space coordinates are $\phi$ and $\chi$. After explicit calculations, similar to pure dilaton case above we obtain the following final expression for $S_{\ln }$, which is relevant to the anomaly,

$$
\begin{aligned}
S_{\ln }= & \frac{1}{16 \pi G} \int d^{4} x \sqrt{-g}\left[l^{3}\left(-\frac{1}{24} R^{2}+\frac{1}{8} R_{i j} R^{i j}\right)+\frac{l^{3}}{4} R^{i j} h_{\mu \nu} \partial_{i} X^{\mu} \partial_{j} X^{\nu}\right. \\
& -\frac{l^{3}}{12} R h_{\mu \nu}\left(\partial X^{\mu} \cdot \partial X^{\nu}\right)-\frac{l^{3}}{24}\left\{h_{\mu \nu}\left(\partial X^{\mu} \cdot \partial X^{\nu}\right)\right\}^{2}+\frac{l^{3}}{8} h_{\mu \nu} h_{\rho \sigma}\left(\partial X^{\mu} \cdot \partial X^{\rho}\right)\left(\partial X^{\nu} \cdot \partial X^{\sigma}\right) \\
& \left.+\frac{l^{8}}{8} h_{\mu \nu}\left\{\triangle X^{\mu}+\Gamma_{\rho \sigma}^{\mu}\left(\partial X^{\rho} \cdot \partial X^{\sigma}\right)\right\}\left\{\triangle X^{\nu}+\Gamma_{\tau \eta}^{\nu}\left(\partial X^{\tau} \cdot \partial X^{\eta}\right)\right\}\right] .
\end{aligned}
$$

Here $\left(\partial X^{\mu} \cdot \partial X^{\nu}\right) \equiv g^{i j} \partial_{i} X^{\mu} \partial_{j} X^{\nu}$ and $\Gamma_{\nu \rho}^{\mu}$ is a connection on the target manifold: $\Gamma_{\nu \rho}^{\mu}=\frac{1}{2} h^{\mu \tau}\left(h_{\nu \tau, \rho}+h_{\rho \tau, \nu}-h_{\nu \rho, \tau}\right)$. If we put

$$
h_{\mu \nu}=\left(\begin{array}{ll}
2 & 0 \\
0 & c
\end{array}\right),(c \text { is an arbitrary constant }), \quad X^{2}=0
$$

the previous result in [167] (see Eq.274) for dilatonic gravity can be reproduced.

Furthermore, if we choose the action as motivated by the bosonic sector of type IIB supergravity with RR-scalar 174

$$
S=\frac{1}{16 \pi G} \int_{M_{d+1}} d^{d+1} x \sqrt{-\hat{G}}\left(\hat{R}+\frac{1}{2}(\hat{\nabla} \phi)^{2}+\frac{1}{2} \mathrm{e}^{2 \phi}(\hat{\nabla} \chi)^{2}\right) .
$$

the result in (275) can be reproduced if we choose

$$
\frac{l^{3}}{16 \pi G}=\frac{2 N^{2}}{(4 \pi)^{2}}, \quad \varphi=\phi+\mathrm{e}^{\phi} \chi, \quad \varphi^{*}=-\phi+\mathrm{e}^{\phi} \chi
$$

and consider the background where only gravity and the complex scalar field $\varphi$ in the $\mathcal{N}=4$ conformal supergravity multiplet are non-trivial and other fields vanish in (275).

Thus, we presented the way to calculate conformal anomaly depending not only from gravitational field but also from other fields via bulk/boundary correspondence. It would be interesting to study the universal structure of holographic conformal anomaly in the presence of dilaton in the same fashion as it is done in ref. 175. 


\subsection{Running gauge coupling and quark-antiquark potential from IIB super- gravity}

AdS/CFT correspondence [164, 165, 176, 163 may also provide new insights to the understanding of non-perturbative QCD. For example, in frames of Type 0 string theory the attempts 177, 178, 179, 180. 181, 182, 183 have been done to reproduce such well-known QCD effects as running gauge coupling and possibly confinement. It is among the first problems to get the description of well-known QCD phenomena from bulk/boundary correspondence.

In another approach one can consider IIB supergravity vacuum which describes the strong coupling regime of a (non)-SUSY gauge theory. This can be achieved by the consideration of deformed IIB SG vacuums, for example, with non-constant dilaton which presumably breaks the conformal invariance and supersymmetry of boundary super YM. Such background will be the perturbation of $\mathrm{AdS}_{5} \times \mathrm{S}_{5}$ vacuum. The background of such sort (with non-trivial dilaton) which interpolates between AdS (UV) and flat space with singular dilaton (IR) has been found in ref. 184.

This solution of IIB SG [184 has been used in ref. 185 with the interpretation of it as the one describing the running gauge coupling (via exponent of dilaton). QCD-like properties of such and similar backgrounds have been discussed in detail in refs. 186, 187, 188, 189, 190, 191, 192]. Modifications of IIB SG solution with non-constant dilaton [184] due to presence of constant self-dual vector 193 or world volume scalar 194 give further indication on the possible confinement and asymptotic freedom of the boundary gauge theory. Unfortunately, situation is very complicated here due to double role of IIB SG backgrounds. From one side they may indeed correspond to IR gauge theory (deformation of initial SUSY YM theory). On the same time such background may simply describe another vacuum of the same maximally supersymmetric YM theory with non-zero VEV of some operator. Due to fact that operators corresponding to deformation to another gauge theory are not known, it is unclear what is the case under discussion (interpretation of SG background). Only some indirect arguments as below may be given. As we see these arguments indicate that IIB SG background discussed in this section most probably correspond to another vacuum of super YM theory under consideration. Then RG flow is induced in the theory via giving a nonzero VEV to some operator.

In this section, we review the set of classical solutions in IIB supergravity and their properties typical for QCD-like boundary gauge theory 195$]$.

Let us make few remarks on AdS/CFT interpretation of IIB SG background. Choosing the coordinates in the asymptotically $\mathrm{AdS}_{5}$ spacetime as

$$
d s^{2}=d \sigma^{2}+S(\sigma) \sum_{i, j=0}^{3} \eta_{i j} d x^{i} d x^{j},
$$

one sees that scalar field $\lambda$, e.g. dilaton, axion or other fields, obey the following equation: $\frac{d^{2} \lambda}{d \sigma^{2}}+4 \frac{d \lambda}{d \sigma}=$ $M^{2} \lambda$. Here $M^{2}$ is the "mass" of $\lambda$ and $\sigma \rightarrow 0$ corresponds to the boundary of AdS. Then $\lambda$ is associated with the operator $\mathcal{O}_{\lambda}$ with conformal dimension $\Delta=2+\sqrt{4+M^{2}}$. The solution of the equation is given by

$$
\lambda=A \mathrm{e}^{-(4-\Delta) \sigma}+B \mathrm{e}^{-\Delta \sigma} .
$$


The solution corresponding to $A$ is not normalizable but the solution to $B$ is normalizable. According to the argument in [196, the non-normalizable solution is associated with the deformation of the $\mathcal{N}=4$ theory by $\mathcal{O}_{\lambda}$ but the normalizable solution is associated with a different vacuum where $\mathcal{O}_{\lambda}$ has a nonzero vacuum expectation value. The behavior of the dilaton is normalizable and seems to be associated with the dimension 4 operator, say $\operatorname{tr} F^{2}$. Then the arguments in 196 would indicate that such solution should correspond to another vacuum of $\mathcal{N}=4$ theory. Nevertheless, there might be still possibility that the solution corresponds to non-SUSY gauge theory. Since there occurs the condensation of $\operatorname{tr} F^{2}$ in the usual non-supersymmetric QCD, in any case, the solution given here describes some features typical for the non-supersymmetric theory.

The situation is even more complicated due to limits of validity of dual SG description. In order that the classical supergravity description is valid, the curvature should be small and the string coupling should be also small. If the curvature is large, the $\alpha^{\prime}$ corrections from string theory would appear. In the AdS/CFT correspondence, the radius $R_{s}$ of the curvature is given by $R_{s}=\left(4 \pi g_{s} N\right)^{\frac{1}{4}}$. Here $g_{s}$ is the string coupling and $N$ is the number of the coincident D-branes. Therefore we should require $g_{s} N \gg 1$. On the other hand, the classical picture works when the string coupling is small: $g_{s} \ll 1$. In the solution given here, there appears the curvature singularity and $g_{s}$ depends on the coordinates since the dilaton is non-trivial. If we concentrate on the behavior near the boundary, which is asymptotically AdS and is far from the singularity, the solution is reliable and SG description may be trusted.

We start from the following action of dilatonic gravity in $d+1$ dimensions:

$$
S=-\frac{1}{16 \pi G} \int d^{d+1} x \sqrt{-G}\left(R-\Lambda-\alpha G^{\mu \nu} \partial_{\mu} \phi \partial_{\nu} \phi\right) .
$$

This action lies in the class of dilatonic gravity models under discussion in this review. In the following, we assume $\lambda^{2} \equiv-\Lambda$ and $\alpha$ to be positive. The action (285) is very general. It contains the effective action of type IIB string theory. The type IIB supergravity, which is the low energy effective action of the type IIB string theory, has a vacuum with only non-zero metric and the anti-self-dual five-form. The latter is given by the Freund-Rubin-type ansatz:

$$
F_{\mu \nu \rho \kappa \lambda}=-\frac{\sqrt{\Lambda}}{2} \epsilon_{\mu \nu \rho \kappa \lambda}(\mu, \nu, \cdots=0,1, \cdots, 4), \quad F_{i j k p q}=-\frac{\sqrt{\Lambda}}{2} \epsilon_{i j k p q}(i, j, \cdots=5, \cdots, 9) .
$$

The vacuum has the topology of $\mathrm{AdS}_{5} \times \mathrm{S}^{5}$. Since $\mathrm{AdS}_{5}$ has a four dimensional Minkowski space as a subspace, especially on its boundary, $\mathrm{AdS}_{5}$ has the four dimensional Poincaré symmetry $\operatorname{ISO}(1,3) . \mathrm{S}^{5}$ has, of course, $S O(6)$ symmetry.

As an extension, we can consider the solution where the dilaton is non-trivial but the anti-selfdual five-form is the same as in (286). Furthermore if we require the solution has the symmetry of $I S O(1,3) \times S O(6)$, the metric should have the following form as in ref. 184 :

$$
d s^{2}=G_{\mu \nu} d x^{\mu} d x^{\nu}+g_{m n} d x^{m} d x^{n}, \quad G_{\mu \nu} d x^{\mu} d x^{\nu}=f(y) d y^{2}+y \sum_{i, j=0}^{d-1} \eta_{i j} d x^{i} d x^{j} .
$$

where $g_{m n}$ is the metric of $S^{5}$. In order to keep the symmetry of $I S O(1,3) \times S O(6)$, the dilaton field $\phi$ can only depend on $y$. Then by integrating five coordinates on $\mathrm{S}^{5}$, we obtain the effective five dimensional 
theory, which corresponds to $d=4$ and $\alpha=\frac{1}{2}$ case in (285). We keep working with above dilatonic gravity as it will be easy to come to IIB supergravity $\left(d=4, \alpha=\frac{1}{2}\right)$ at any step.

The equations of motion given by the variation of the action (285) with respect to the metric $G^{\mu \nu}$ and the dilaton $\phi$ can be exactly solved if we assume that $\phi$ depends only on one of the coordinate, say $y \equiv x^{d}$ as in type IIB supergravity solution with the symmetry of $I S O(1,3) \times S O(6)$ and we can also consider, as a generalization of 287, , the case that $\eta_{i j}$ in (287) is replaced by $g_{i j}$, which is the metric in the Einstein manifold defined by $r_{i j}=k g_{i j}$. Here $r_{i j}$ is the Ricci tensor given by $g_{i j}$ and $k$ is a constant, especially $k>0$ for sphere and $k=0$ for the flat Minkowski space and $k<0$ for hyperboloid. The case of $k=1$ is especially interesting as it corresponds to gauge theory in de Sitter (inflationary) Universe. The solution is given by

$$
f=\frac{d(d-1)}{4 y^{2} \lambda^{2}\left(1+\frac{\alpha c^{2}}{\lambda^{2} y^{d}}+\frac{k d}{\lambda^{2} y}\right)}, \quad \phi=c \int d y \sqrt{\frac{d(d-1)}{4 y^{d+2} \lambda^{2}\left(1+\frac{\alpha c^{2}}{\lambda^{2} y^{d}}+\frac{k d}{\lambda^{2} y}\right)}} .
$$

From the behavior of $f(y)$, we find that there is a curvature singularity at $y=0$. The curvature singularity would be generated by the singular behavior of the dilaton $\phi$ when $y \sim 0$. The curvature singularity tells there should appear the $\alpha^{\prime}$ correction from the string theory and the supergravity description would break down when $y \sim 0$. Conversely and hopefully, the curvature singularity might be apparent and vanish when we can include full string corrections. In any case, the dual interpretation of the solution can be trusted if we investigate the behavior near the boundary $(y \rightarrow+\infty)$.

If we change the coordinate $y$ by $\rho$ in (288), which is defined by

$$
\rho \equiv-\int d y \sqrt{\frac{f(y)}{y}}=-\int d y \sqrt{\frac{d(d-1)}{4 y^{3} \lambda^{2}\left(1+\frac{\alpha c^{2}}{\lambda^{2} y^{d}}+\frac{k d}{\lambda^{2} y}\right)}},
$$

the metric has the following form

$$
G_{\mu \nu} d x^{\mu} d x^{\nu}=\Omega^{2}(\rho)\left(d \rho^{2}+\sum_{i, j=0}^{d-1} g_{i j} d x^{i} d x^{j}\right) .
$$

Here $\Omega^{2}(\rho)$ is given by solving $y$ in (289) with respect to $\rho$ : $\Omega^{2}(\rho)=y(\rho)$. When $\rho$ is small, $y$ is large and the structure of the spacetime becomes AdS asymptotically. From (289), we find $\rho=$ $\frac{\sqrt{d(d-1)}}{\lambda y^{\frac{1}{2}}}\left(1+\mathcal{O}\left(y^{-1}\right)\right)$. Therefore we find

$$
\Omega^{2}(\rho)=y(\rho)=\frac{R_{s}^{2}}{\rho^{2}}\left(1+\mathcal{O}\left(\rho^{2}\right)\right), \quad R_{s} \equiv \frac{\sqrt{d(d-1)}}{\lambda} .
$$

The $\mathrm{AdS}_{5}$ part in the solution has the form (properly normalized)

$$
d s_{\mathrm{AdS}_{5}}^{2}=\left(4 \pi g_{s} N\right)^{\frac{1}{2}} \cdot \frac{1}{\rho^{2}}\left(d \rho^{2}+\sum_{i, j=0}^{d-1} \eta_{i j} d x^{i} d x^{j}\right)
$$

Therefore we find

$$
R_{s}=\left(4 \pi g_{s} N\right)^{\frac{1}{4}}
$$


where $g_{s}$ is the string coupling and $N$ is the flux of the five-form $F$ in (286) through $\mathrm{S}^{5}$, which is produced by the $N$ coincident D3-branes.

It is interesting that there are many Einstein manifolds which solve the model. The Einstein equations are given by,

$$
R_{\mu \nu}-\frac{1}{2} g_{\mu \nu} R+\frac{1}{2} \Lambda g_{\mu \nu}=T_{\mu \nu}^{\text {matter }}
$$

Here $T_{\mu \nu}^{\text {matter }}$ is the energy-momentum tensor of the matter fields. If we consider the vacuum solution where $T_{\mu \nu}^{\text {matter }}=0$, Eq. (294) can be rewritten as $R_{\mu \nu}=\frac{\Lambda}{2} g_{\mu \nu}$. If we put $\Lambda=2 k$, this is nothing but Eq. for the Einstein manifold. The Einstein manifolds are not always homogeneous manifolds like flat Minkowski, (anti-)de Sitter space or Nariai space but they can be some black hole solutions like Schwarzschild black hole,

$$
d s_{4}^{2} \equiv \sum_{\mu, \nu=0}^{3} g_{\mu \nu} d x^{\mu} d x^{\nu}=-\left(1-\frac{r_{0}}{r}\right) d t^{2}+\frac{d r^{2}}{\left(1-\frac{r_{0}}{r}\right)}+r^{2} d \Omega^{2},
$$

or Kerr one for $k=0^{6}$ or Schwarzschild (anti-) de Sitter black hole for $k \neq 0$. In these solutions, the curvature singularity at $r=0$ has a form of line penetrating $\mathrm{AdS}_{5}$ and the horizon makes a tube surrounding the singularity. This configuration seems to express D-string whose boundary lies on the boundary of $\mathrm{AdS}_{5}$ or possibly D3-brane. Especially in case of Kerr or Kerr-(anti-)de Sitter solution, the object corresponding to the singularity has an angular momentum.

Consider the running of the gauge coupling, which is given by the dilaton field $\phi$ due to AdS/CFT correspondence. We assume the gauge coupling has the following form

$$
g=g_{s} \mathrm{e}^{2 \beta \sqrt{\frac{\alpha}{d(d-1)}}\left(\phi-\phi_{0}\right)}=g_{s}\left\{1-\frac{2 \beta c \sqrt{\alpha}}{d \lambda} y^{-\frac{d}{2}}+\frac{k d \beta c \sqrt{\alpha}}{(d+2) \lambda^{3}} y^{-\frac{d}{2}-1}+\cdots\right\} .
$$

In case of type IIB supergravity $\left(\alpha=\frac{1}{2}\right), \beta=\sqrt{\frac{d(d-1)}{2}}$. If we define a new coordinate $U$ by $y=U^{2}, U$ expresses the scale on the (boundary) $d$ dimensional space (due to holography 198, 199]). Following the correspondence between long distances/high energy in the AdS/CFT scheme, $U$ can be regarded as the energy scale of the boundary field theory. Then we obtain the following renormalization group equation

$$
\begin{aligned}
& \circ k \neq 0 \quad \beta(U) \quad \equiv U \frac{d g}{d U}=-d\left(g-g_{s}\right)-\frac{2 k d \beta c \sqrt{\alpha}}{(d+2) \lambda^{3}}\left(\frac{d \lambda}{2 \beta c \sqrt{\alpha}}\right)^{\frac{2}{d}+1} \frac{\left(g-g_{s}\right)^{\frac{2}{d}+1}}{g_{s} \frac{2}{d}}+\cdots \\
& \circ k=0 \quad \beta(U)=-d\left(g-g^{*}\right)-\left(d-\frac{d^{2}}{2 \beta}\right) \frac{\left(g-g_{s}\right)^{2}}{g_{s}}+\cdots
\end{aligned}
$$

The leading behavior is the same as in refs. [185, 186, 187, 188, 193, 195] and tells that QFT on the boundary has the non-trivial UV-fixed point.

Hence, we found that beta-function explicitly depends on the curvature of four-dimensional manifold. Of course, curvature dependence is not yet logarithmic as it happens with usual QFTs (perturbative consideration) in curved spacetime 21. The power-like running of gauge coupling is much stronger than in $k=0$ case. Note that previous discussion of power-like running includes GUTs with large internal dimensions 200, 201, 202, 203, 204, 205.

\footnotetext{
${ }^{6}$ This type of solutions for $k=0$ case has been considered in ref. [197]
} 
One may also comment about the static potential between "quark" and "anti-quark" 206]. We evaluate the following Nambu-Goto action

$$
S=\frac{1}{2 \pi} \int d \tau d \sigma \sqrt{\operatorname{det}\left(g_{\mu \nu}^{s} \partial_{\alpha} x^{\mu} \partial_{\beta} x^{\nu}\right)} .
$$

with the "string" metric $g_{\mu \nu}^{s}$, which could be given by multiplying a dilaton function $k(\phi)$ to the metric tensor in (287). Especially we choose $k(\phi)$ by

$$
k(\phi)=\mathrm{e}^{2 \gamma \sqrt{\frac{\alpha}{d(d-1)}}\left(\phi-\phi_{0}\right)}=1-\frac{2 \gamma c \sqrt{\alpha}}{d \lambda y^{\frac{d}{2}}}+\cdots
$$

In case of type IIB supergravity, $\gamma=\beta=\sqrt{\frac{d(d-1)}{2}}$. We consider the static configuration $x^{0}=\tau$, $x^{1} \equiv x=\sigma, x^{2}=x^{3}=\cdots=x^{d-1}=0$ and $y=y(x)$. The orbit of $y$ can be obtained by minimizing the action $S$ or solving the Euler-Lagrange equation $\frac{\delta S}{\delta y}-\partial_{x}\left(\frac{\delta S}{\delta\left(\partial_{x} y\right)}\right)=0$. Substituting the configuration into the action (298), we obtain the total energy of the system $E(L)$ by $S=\frac{T}{2 \pi} E(L)$. Here $T$ is the length of the region of the definition of $\tau$. The energy $E(L)$, however, contains the divergence due to the self energies of the infinitely heavy "quark" and "anti-quark". The sum of their self energies can be estimated by considering the configuration $x^{0}=\tau, x^{1}=x^{2}=x^{3}=\cdots=x^{d-1}=0$ and $y=y(\sigma)$ (note that $x_{1}$ vanishes here) and the minimum of $y$ at $y_{D}$ where brane would lie. Then the finite potential $E_{q \bar{q}}(L) \equiv E(L)-E_{\text {self }}$ between "quark" and "anti-quark" is given by

$$
\begin{aligned}
& \circ k \neq 0 \quad E_{q \bar{q}}(L)=\frac{1}{A}\left(\frac{C_{\frac{3}{2}}}{A L}\right)\left\{D_{0}+B\left(\frac{C_{\frac{5}{2}} D_{0}}{C_{\frac{3}{2}}}+D_{2}\right)\left(\frac{A L}{C_{\frac{3}{2}}}\right)^{2}+\cdots\right\} \\
& A \equiv \frac{2 \lambda}{\sqrt{d(d-1)}}, \quad B \equiv-\frac{k d \gamma}{4 \lambda^{2}}, \quad C_{a} \equiv \int_{-\infty}^{\infty} d t \cosh ^{-a} t=\frac{2^{(a-1)} \Gamma\left(\frac{a}{2}\right)^{2}}{\Gamma(a)} \\
& D_{d} \equiv 2 \int_{0}^{\infty} d t \cosh ^{-\frac{d+1}{2}} t \mathrm{e}^{-t}+\frac{4}{d-1}=\frac{2^{\frac{d-3}{2}} \Gamma\left(\frac{d-1}{4}\right)^{2}}{\Gamma\left(\frac{d-1}{2}\right)} \\
& \circ k=0 \quad E_{q \bar{q}}(L)=\left(\frac{C_{\frac{3}{2}}}{A L}\right)\left\{D_{0} \pm B_{0}\left(D_{d}+F_{d}+\frac{S_{d} D_{0}}{C_{\frac{3}{2}}}\right)\left(\frac{A L}{C_{\frac{3}{2}}}\right)^{d}+\mathcal{O}\left(L^{2 d}\right)\right\} \\
& S_{d} \equiv \int_{-\infty}^{\infty} d t \cosh ^{\frac{1}{2}} t \sinh ^{-2} t\left(1-\cosh ^{-\frac{d}{2}} t\right), F_{d} \equiv \int_{-\infty}^{\infty} d t \sinh ^{-2} t \cosh ^{\frac{1}{2}} t\left(1-\cosh ^{-\frac{d}{2}} t\right) .
\end{aligned}
$$

Here $L$ is the distance between "quark" and "anti-quark" and we neglected the $L$ independent terms. Note that leading and next-to-leading term for $k \neq 0$ does not depend on the parameter $\gamma$ in (299). The leading behavior is attractive since $D_{0}=-2.39628 \ldots$ but we should note that next-to-leading term is linear in $L$ for $k \neq 0$, which does not depend on the dimension $d$. Since $\frac{C_{\frac{5}{2}} D_{0}}{C_{\frac{3}{2}}}+D_{2}=3.49608>0$ and $B$ is negative if $k$ is positive and vice versa. Therefore the linear potential term in (300) is repulsive if $k>0$ (sphere, i.e. gauge theory in de Sitter Universe) and attractive if $k<0$ (hyperboloid).

Of course, the confinement depends on the large $L$ behavior of the potential. When $L$ is large, however, the orbit of the string would approach to the curvature singularity at $y=0$, where the supergravity description would break down. Despite of this, there might be interesting to investigate the large $L$ behavior. From the behavior of $f(y)$ and the dilaton $\phi$ when $y$ is small, we find the integrand in the 
Nambu-Goto action behaves as

$$
\begin{aligned}
& k(\phi(y)) y \sqrt{\frac{f(y)}{y}\left(\partial_{x} y\right)^{2}+1} \sim y^{\operatorname{sgn}(c) \sqrt{\frac{d(d-1)}{2}}+1} \sqrt{\frac{d(d-1)}{4 \alpha c^{2}} y^{d-3}\left(\partial_{x} y\right)^{2}+1} \\
& =\sqrt{\frac{d(d-1)}{4 \alpha c^{2}\left(\operatorname{sgn}(c) \sqrt{\frac{d(d-1)}{2}}+\frac{d+1}{2}\right)^{2}}\left(\partial_{x} \tilde{U}\right)^{2}+(\tilde{U})^{\gamma_{0}}} .
\end{aligned}
$$

Here $\tilde{U} \equiv y^{\operatorname{sgn}(c) \sqrt{\frac{d(d-1)}{2}}+\frac{d+1}{2}}$ and $\gamma_{0} \equiv \frac{2 \operatorname{sgn}(c) \sqrt{\frac{d(d-1)}{2}}+2}{\operatorname{sgn}(c) \sqrt{\frac{d(d-1)}{2}}+\frac{d+1}{2}}$. When $d=4,0<\gamma_{0}<2$ when $c>0$ and $\gamma<0$ when $c<0$. According to the analysis in 196, the orbit of the string goes straight to the region $y \sim 0$ when $c>0\left(0<\gamma_{0}<2\right)$ and the potential becomes independent of $L$. In this case, however, the potential would receive the $\alpha^{\prime}$ correction from the string theory. On the other hand, when $c<0(\gamma<0)$, there is an effective barrier which prevents the orbit of string from approaching into the curvature singularity and the potential would not receive the $\alpha^{\prime}$ correction so much and the supergravity description would be reliable. Furthermore $c<0(\gamma<0)$ case predicts the confinement. Similarly, one can evaluate the potential between monopole and anti-monopole by using the Nambu-Goto action for $D$-string instead of (298) (cf.ref.207) and study its behavior.

One can include the axion field $\chi$ into the action of type IIB supergravity $\left(\alpha=\frac{1}{2}\right)$ in (285), following ref. 174,208

$$
S=-\frac{1}{16 \pi G} \int d^{d+1} x \sqrt{-G}\left(R+\lambda^{2}-\frac{1}{2} G^{\mu \nu} \partial_{\mu} \phi \partial_{\nu} \phi+\frac{1}{2} \mathrm{e}^{2 \phi} G^{\mu \nu} \partial_{\mu} \chi \partial_{\nu} \chi\right) .
$$

Again the solution may be found and its boundary gauge theory interpretation presented. By properly choosing the constants of the integration, the boundary field theory can be regarded as asymptotically free. We also find the dilaton field behaves, when $y$ is small as $\phi \sim-\sqrt{\frac{d(d-1)}{2}} \ln y$, which corresponds to $c<0$ case in the pure dilaton case. Since the behavior of the metric is essentially identical with the pure dilaton case, the supergravity description would be valid even for large $L$ and the confinement would be predicted. Especially, one can investigate the supersymmetric background. Since that is background where the fermion fields, that is, dilatino $\xi$ and gravitino $\psi_{\mu}$ vanish, if the variation under some of the supersymmetry transformations of these fermionic fields vanishes the corresponding supersymmetries are preserved. We find that although all supersymmetries break down in general, half of the supersymmetries survives. This situation does not depend on $k$. Such solution corresponds to super YM theory with asymptotically free gauge coupling and two supersymmetries broken. In the same way, one can study dilatonic AdS-like backgrounds in the presence of other fields, like fermionic ones. Our next problem will be to take into account temperature in the above picture.

\subsection{Dilatonic AdS black holes: running gauge coupling and $q \bar{q}$-potential at non-zero temperature}

It has been realized that planar $\mathrm{AdS}_{5} \mathrm{BH}$ is dual to a thermal state of $\mathcal{N}=4$ super YM theory. The corresponding coupling constant dependence has been studied in ref. 209, 210 based on earlier study of SG side free energy in ref. 211. Spherical AdS BH shows the finite temperature phase transition 
212 which may be used to realize the confinement in large $N$ theory at low temperatures [165]. In this section, based on [213], we give the example of the deformation of IIB SG $\mathrm{AdS}_{5} \times \mathrm{S}_{5}$ background with non-trivial dilaton where AdS sector is described by $\mathrm{BH}$ (hence, temperature appears). Then, running gauge coupling of gauge theory at non-zero temperature may be defined by exponent of dilaton. We give some approximate solutions of IIB SG] with such properties where running coupling shows power-like behavior in the temperature (in the expansion on radius).

We start from the action of dilatonic gravity in $d+1$ dimensions in (285). We assume the $(d+1)$ dimensional metric is given by

$$
d s^{2}=-\mathrm{e}^{2 \rho} d t^{2}+\mathrm{e}^{2 \sigma} d r^{2}+r^{2} \sum_{i, j=1}^{d-1} g_{i j} d x^{i} d x^{j} .
$$

Here $g_{i j}$ does not depend on $r$ and it is the metric in the Einstein manifold. We also introduce new variables $U$ and $V$ by

$$
U \equiv \mathrm{e}^{\rho+\sigma}, \quad V \equiv r^{d-2} \mathrm{e}^{\rho-\sigma}
$$

When the dilaton vanishes (or constant), the solution is given by

$$
U=1, \quad V=V_{0} \equiv \frac{k r^{d-2}}{d-2}+\frac{\lambda^{2}}{d(d-1)} r^{d}-\mu
$$

Here $\mu$ corresponds to the mass of the black hole. $k=0$, positive or negative corresponds to planar, spherical or hyperbolic AdS BH, respectively. When $\mu=0$, the solution is isomorphic to AdS. If we choose $k<0$, the metric has the following form:

$$
d s^{2}=-\frac{\left(r^{2}-r_{0}^{2}\right)}{l^{2}} d t^{2}+\frac{l^{2}}{\left(r^{2}-r_{0}^{2}\right)} d r^{2}+r^{2} \sum_{i, j=1}^{d-1} g_{i j} d x^{i} d x^{j}
$$

Here

$$
l^{2} \equiv \frac{d(d-1)}{\lambda^{2}}, \quad r_{0} \equiv l \sqrt{-\frac{k}{d-2}} .
$$

The obtained AdS metric has a horizon at $r=r_{0}$. From the behavior of the metric at $r \sim r_{0}$ after Wick-rotated, we find a temperature $T$ :

$$
T=\frac{r_{0}}{2 \pi l^{2}}=\frac{1}{2 \pi l} \sqrt{\frac{-k}{d-2}} .
$$

One can now consider the perturbation by assuming the dilaton is small 213 . We will concentrate on the case of type IIB SG in $d=4$, by putting $\alpha=\frac{1}{2}$. Note that in this approximation the radius is away from horizon. Near-horizon regime may be discussed independently.

For $\mu=0$ and $k<0$ case, the leading term for the dilaton $\phi$ is is found as

$$
\begin{aligned}
\phi & =\phi_{0}+c l^{2}\left\{\frac{1}{2 r_{0}^{4}} \ln \left(1-\frac{r_{0}^{2}}{r^{2}}\right)+\frac{1}{2 r_{0}^{2} r^{2}}\right\} \\
& =\phi_{0}+c\left\{\frac{1}{2 l^{6}(2 \pi T)^{4}} \ln \left(1-\frac{l^{4}(2 \pi T)^{2}}{r^{2}}\right)+\frac{1}{2 l^{2}(2 \pi T)^{2} r^{2}}\right\} .
\end{aligned}
$$

\footnotetext{
${ }^{7}$ These solutions presumably describe thermal states of (non)-SUSY gauge theory which descends from $\mathcal{N}=4$ super YM after breaking of SUSY and conformal invariance or another vacuum of the same super YM theory.
} 
which gives the temperature dependent running dilaton. We should note that there is a singularity in the dilaton field at the horizon $r=r_{0}=2 \pi l^{2} T$. The fact that dilaton may become singular at IR has been mentioned already in two-boundaries AdS solution of IIB SG in ref. 184. It is also interesting that when $r$ is formally less than $r_{0}$ then dilaton (and also running coupling) becomes imaginary.

Since the string coupling is given by $g=g_{s} \mathrm{e}^{\phi}\left(g_{s}\right.$ : constant), we find the behavior of $g$ when $r$ is large and $c$ is small. Since $r$ is the length scale corresponding to the radius of the boundary manifold, $r$ can be regarded as the energy scale of the field theory on the boundary [198, 199]. Therefore the beta-function is given by 213

$$
\beta(g)=r \frac{d g}{d r}=-4\left(g-g_{s}\right)+\frac{2^{\frac{5}{2}}}{3}(2 \pi T)^{2} l^{3} g_{s}\left(\frac{g_{s}-g}{c g_{s}}\right)^{\frac{3}{2}} .
$$

The first term is usual and universal. The second term defines the temperature dependence.

Let us comment on the case of high $T$. As we consider the behavior near the boundary, first we take $r$ to be large. After that we consider the case of high $T$. In this case $r \gg T l^{2}$ and we can consider the large $T$ case in the expression (310). The problem might happen when $r \sim T l^{2}$. In this case, we need to solve Eq.(309) with respect to $r$ as a function of $T$ and $\phi$ or coupling: $r=r(g, T)$. Then from (309), we find the following expression of the beta-function:

$$
\left.\beta(g) \sim r \frac{d g}{d r}\right|_{r=r(g, T)}=\frac{g_{s} c l^{2}}{r(g, T)^{4}\left(1-\frac{l^{4}(2 \pi T)^{2}}{r(g, T)^{2}}\right)} .
$$

In case $r$ is large, the above equation reproduces (310). We can also consider the case that the last term in (309) is larger than the second term which contains $\ln (\cdots)$. In this case, the leading behavior of the beta-function has the following form: $\beta(g) \sim-2\left(g-g_{s}\right)+\cdots$. This beta-function presumably defines strong coupling regime of non-SUSY gauge theory at high temperature. It is interesting to note that in perturbative gauge theory at non-zero temperature the running gauge coupling contains not only standard logarithms of $T$ but also terms linear on $T$ (see ref.214] and references therein). Of course, in our case we have not asymptotically free theory but the one with stable fixed point.

Now we consider the correction for $V$ and $U$, writing them in the following form: $V=V_{0}+c^{2} v$, $U=1+c^{2} u$, where $v=v(s), u=u(s)$ are given explicitly in 213. Here $s=-\frac{2 r^{2}}{k l^{2}}$. Then we find that $U$ and $V$ or $\mathrm{e}^{2 \rho}$ and $\mathrm{e}^{2 \sigma}$ have the singularity at the non-perturbed horizon corresponding to $s=1$. Eq.(309) tells also that the dilaton field is also singular there. In other words, the expansion with respect to $c^{2}$ breaks down when $s \sim 0$. Therefore the singularity in $U, V$ would not be real one.

In order to investigate the behavior in near-horizon regime we assume that the radius of the horizon is large and use $\frac{1}{r}$ expansion: $V=\frac{r^{4}}{l^{2}}+\frac{k r^{2}}{2}+\frac{a}{r^{4}}+\mathcal{O}\left(r^{-6}\right)$. We put the constant term to be zero assuming that the black hole mass vanishes. Then $a=\frac{c^{2} l^{2}}{48}$ and $\mathrm{e}^{\phi}, V$ and $U$ have the following forms:

$$
\begin{aligned}
& \mathrm{e}^{\phi}=\mathrm{e}^{\phi_{0}}\left(1-\frac{c l^{2}}{4 r^{4}}+\mathcal{O}\left(r^{-6}\right)\right) \\
& V=\frac{r^{4}}{l^{2}}+\frac{k r^{2}}{2}+\frac{c^{2} l^{2}}{48 r^{4}}+\mathcal{O}\left(r^{-6}\right), \quad U=1-\frac{c^{2} l^{4}}{192 r^{8}}+\mathcal{O}\left(r^{-10}\right) .
\end{aligned}
$$

From the equation $V=0$ we find the position of the horizon and the correction to the temperature:

$$
r=r_{h} \equiv l \sqrt{-\frac{k}{2}}\left(1-\frac{c^{2}}{6 k^{4} l^{4}}\right), \quad T=\frac{1}{2 \pi l} \sqrt{-\frac{k}{2}}-\frac{c^{2}\left(-\frac{k}{2}\right)^{-\frac{7}{2}}}{192 l^{5}} .
$$


We can also analyze the potential between quark and anti-quark 206 by evaluating the Nambu-Goto action in (298) as in the previous section. We consider the static configuration $x^{0}=\tau, x^{1} \equiv x=\sigma$, $x^{2}=x^{3}=\cdots=x^{d-1}=0$ and $r=r(x)$. Choose the coordinates on the boundary manifold so that the line given by $x^{0}=$ constant, $x^{1} \equiv x$ and $x^{2}=x^{3}=\cdots=x^{d-1}=0$ is geodesic and $g_{11}=1$ on the line. The orbit of $r$ can be obtained by minimizing the action $S$ or solving the Euler-Lagrange equation. By the similar procedure in the previous section, we find the following expression for quark-antiquark potential 213

$$
\begin{aligned}
E_{q \bar{q}}(L) & =\frac{l A C}{L}-\frac{l^{3}(2 \pi T)^{2}}{2}\left(\frac{B C}{A^{2}}+\frac{D}{A}\right) L+\mathcal{O}\left(L^{3}\right) \\
C & =2 \int_{0}^{\infty} d t\left\{\frac{\cosh ^{2} t}{\left(\cosh ^{2} t+1\right)^{\frac{1}{2}}}-\sinh t\right\}-2=-1.19814 \ldots \\
D & =2 \int_{0}^{\infty} \frac{d t}{\left(\cosh ^{2} t+1\right)^{\frac{3}{2}}}=0.711959 .
\end{aligned}
$$

Here we neglected the $L$ independent term. We should note that next-to-leading term is linear in $L$, which might be relevant to the confinement. For the confinement, it is necessary that the quark-antiquark potential behaves as $E_{q \bar{q}} \sim a L$ with some positive constant $a$ for large $L$. For high temperature, it is usually expected that there occurs the phase transition to the deconfinement phase, where the potential behaves as Coulomb force, $E_{q \bar{q}} \sim \frac{a^{\prime}}{L}$. Since $\frac{B C}{A^{2}}+\frac{D}{A}>0$ and $k<0$, the potential is repulsive. The leading term expresses the repulsive but shows the Coulomb like behavior. The next-leading-term tells that the repulsive force is long-range than Coulomb force.

The expression (314) is correct even at high temperature if $L$ is small. If $L$ is large and the orbit of string approaches to the horizon and/or enters inside the horizon, the expression would not be valid. Since the horizon is given by (307), the expression (314) would be valid if $L \ll A \sqrt{-\frac{2}{k}}=2 \pi A l T$. The above condition makes difficult to evaluate the potential quantitively by the analytic calculation when $L$ is large and numerical calculation would be necessary. In order to investigate the qualitive behavior of the potential when $L$ is large, we consider the background where the dilaton is constant $\phi=\phi_{0}$, which would tell the effect of the horizon or finite temperature. As $c=0$ when the dilaton is constant, we can use the solution in (305). Then by the calculation similar to (314) but without assuming $L$ is small, we obtain the following expression of the quark-antiquark potential:

$$
E_{q \bar{q}}=r_{\min } \int_{-\infty}^{\infty} d t \sinh t\left\{\left(1-\frac{1}{\cosh ^{2} t} \cdot \frac{1-\frac{r_{0}^{2}}{r_{\min }^{2}}}{\cosh ^{2} t-\frac{r_{0}^{2}}{r_{\min }^{2}}}\right)^{-\frac{1}{2}}-1\right\}+2\left(r_{D}-r_{\min }\right) .
$$

Here $r_{\min }$ is the minimum of $r$ and constant -1 in \{\} and the last term correspond to the subtraction of the self-energy. The integration in (315) converges and the integrand is monotonically decreasing function of $\frac{1}{r_{\min }}$ if $r_{\min }$ is larger than the radius of the horizon $r_{0}: r_{\min }>r_{0}$ and vanishes in the limit of $r_{\min } \rightarrow r_{0}$. Therefore if $r_{\min }$ decreases and approaches to $r_{0}$ when $L$ is large, which seems to be very natural, the potential $E_{q \bar{q}}$ approaches to a constant $E_{q \bar{q}} \rightarrow 2\left(r_{D}-r_{0}\right)$ and does not behave as a linear function of $L$ [213. This tells that the quark is not confined. This effect would correspond to deconfining phase of QCD in the finite temperature. 
Similarly, one can consider another interesting case like that $k=0$ and $\mu \neq 0$ [213], which corresponds to the throat limit of D3-brane 209, 211. In a way similar to $k<0$ and $\mu=0$ case, the corrections coming from the dilaton were calculated for the beta-function and quark-antiquark potential with the indication to possible confinement [213]. One can consider even more general case where either $k$ or $\mu$ do not vanish (for details, see [213). The analysis of this case may be done in the same way as above.

It is also extremely interesting to study thermodynamics of obtained dilatonic AdS BHs. After Wickrotating the time variable by $t \rightarrow i \tau$, the free energy $F$ can be obtained from the action $S$ in (285) where the classical solution is substituted after the Wick-rotation. The expression of $S$, however, contains the divergence coming from large $r$. In order to subtract the divergence, we regularize $S$ by cutting off the integral at a large radius $r_{\max }$. After that we subtract the divergent part. In case of $k<0$ and $\mu=0$, we subtract it by using the extremal solution with $c=0$. Then we obtain the following expression 213]

$$
F=-\frac{V_{3}}{2 \pi G l^{2}}\left(\frac{5 l^{8}(2 \pi T)^{4}}{32}+\frac{c^{2}}{768 l^{4}(2 \pi T)^{4}}\right) .
$$

In order to get the entropy $\mathcal{S}$, we need to know $T$ dependence of $V_{3}$ although $V_{3}$ is infinite. Since $k$ is proportional the curvature, $V_{3}$ would be proportional to $k^{-\frac{3}{2}}$. Therefore the entropy $\mathcal{S}$ and mass (energy) $E$ are given by

$$
\begin{aligned}
& \mathcal{S}=-\frac{d F}{d T}=\frac{V_{3}}{2 \pi G l^{2} T}\left(\frac{25 l^{8}(2 \pi T)^{4}}{64}+\frac{49 c^{2}}{1536 l^{4}(2 \pi T)^{4}}\right) \\
& E=F+T \mathcal{S}=\frac{V_{3}}{2 \pi G l^{2}}\left(\frac{15 l^{8}(2 \pi T)^{4}}{64}+\frac{47 c^{2}}{1536 l^{4}(2 \pi T)^{4}}\right) .
\end{aligned}
$$

In terms of string theory correspondence 209, the parameters $G$ and $l$ are given by $l^{4}=2 g_{Y M}^{2} N \alpha^{\prime 2}$ and $G l=\frac{\pi g_{Y M}^{2} \alpha^{\prime 2}}{N}$. Here the Yang-Mills coupling $g_{Y M}$ is given by the string coupling $g_{s}: g_{Y M}^{2}=2 \pi g_{s}$ and $N$ is the number of the coincident D3-branes. As $V_{3}$ is now dimensionless, we multiply $l^{3}$ with $V_{3}$ : $\tilde{V}_{3} \equiv l^{3} V_{3}$. Then Eqs. (316) and (317) can be rewritten as follows:

$$
\begin{aligned}
F & =-\frac{\tilde{V}_{3}}{2 \pi^{2}}\left(\frac{5 N^{2}(2 \pi T)^{4}}{16}+\frac{c^{2}}{3072 l^{4} g_{Y M}^{6} N{\alpha^{\prime}}^{6}(2 \pi T)^{4}}\right) \\
\mathcal{S} & =\frac{V_{3}}{2 \pi^{2} T}\left(\frac{25 N^{2}(2 \pi T)^{4}}{32}+\frac{49 c^{2}}{6144 g_{Y M}^{6} N{\alpha^{\prime}}^{6}(2 \pi T)^{4}}\right) \\
E & =\frac{V_{3}}{2 \pi G l^{2}}\left(\frac{15 N^{2}(2 \pi T)^{4}}{32}+\frac{47 c^{2}}{6144 g_{Y M}^{6} N \alpha^{\prime 6}(2 \pi T)^{4}}\right) .
\end{aligned}
$$

For $k=0$ and $\mu>0$ case, we can obtain the thermodynamical quantities in a similar way, where we regularize $S$ by subtracting the solution with $\mu=0$ and $c=0$. The results are given in [213]. The leading behavior of $F$ and $S$ are consistent with [209]. As we used $\frac{1}{r}$ expansion, the next to leading terms become dominant when the radius of horizon $r_{h}$ is large and the parameter $c$ is not very small. One has to remark that leading term in above free energy describes the strong coupling regime free energy for $\mathcal{N}=4$ super YM theory with the usual mismatch factor $3 / 4$ if compare with perturbative free energy.

Thus, we discussed applications of dilatonic AdS gravity for construction of dilatonic AdS BHs which may be used for description of gauge theory at non-zero temperature. 


\subsection{Strong coupling limit of $\mathcal{N}=2$ superconformal field theory free energy from AdS black hole thermodynamics}

In the previous section we gave one example where AdS BH gives the strong coupling limit of $\mathcal{N}=4$ super YM theory free energy [211, 209] which differs by factor 3/4 with perturbative result (boundary QFT) in the leading order of $1 / N$ expansion. Note that thermodynamics of super YM theory in relation with AdS/CFT correspondence has been discussed in large number of works 215, 210, 216, 217, 181, 218, 219. 220, 221, 222, 223, 224, 225, 226, 227, 228, 229, 230, 213, 231, 232, 233, 234 (see also references therein). It is quite interesting to understand what happens in the next order of $1 / N$ expansion. Clearly that such analysis should be related with higher derivatives (HD) terms on SG side. In more general framework HD terms may help in better understanding of AdS/CFT correspondence or even in formulation of new versions of bulk/boundary conjecture.

That is the purpose of this section to show the role of HD terms in bulk action in AdS/CFT correspondence as it was done in 235. The conformal anomaly of $d=4, \mathcal{N}=2$ and $\mathcal{N}=4$ SCFTs (bulk side calculation) has been found up to next to leading order in the $1 / N$ expansion in refs. 171, 172]. Even in next to leading order term it coincides with QFT result (for gravity side derivation of trace anomaly, mainly in $\mathcal{N}=4$ super YM case, see also refs. 167, 236, 237, 238, 239, 240]). The $\mathcal{N}=2$ theory with the gauge group $S p(N)$ arises as the low-energy theory on the world volume on $N$ D3-branes sitting inside 8 D7-branes at an O7-plane [241, 242, 243, 244, 245. The string theory dual to this theory has been conjectured to be type IIB string theory on $A d S_{5} \times X^{5}$ where $X_{5}=S^{5} / Z_{2}$ [246], whose low energy effective action is given by

$$
S=\int d^{5} x \sqrt{g}\left\{\frac{N^{2}}{4 \pi^{2}}(R+12)+\frac{6 N}{24 \cdot 16 \pi^{2}} R_{\mu \nu \rho \sigma} R^{\mu \nu \rho \sigma}\right\} .
$$

The overall factor of the action is different by $\frac{1}{2}$ from that of the action which corresponds to $\mathcal{N}=4$ $S U(N)$ gauge theory. The latter action is given by type IIB (compactified) string theory on $A d S_{5} \times S^{5}$. The factor $\frac{1}{2}$ comes from the fact that the volume of $X^{5}=S^{5} / Z_{2}$ is half of $S^{5}$ due to $Z_{2}$. Note that Riemann curvature squared term in the above bulk action is deduced from heterotic string via heterotictype I duality [247] (dilaton is assumed to be constant). The interesting fact about theory (319) is that it may be rewritten also as dilatonic gravity.

Then the equations of motion have the following form:

$$
0=-\frac{c}{2} g_{\zeta \xi}\left(R_{\mu \nu \rho \sigma} R^{\mu \nu \rho \sigma}+\frac{1}{\kappa^{2}} R-\Lambda\right)+c R_{\zeta \mu \nu \rho} R_{\xi}^{\mu \nu \rho}+\frac{1}{\kappa^{2}} R_{\zeta \xi}-4 c D_{\nu} D_{\mu} R_{\zeta \xi}^{\mu \nu} .
$$

Here $\frac{1}{\kappa^{2}}=\frac{N^{2}}{4 \pi^{2}}, c=\frac{6 N}{24 \cdot 16 \pi^{2}}, \Lambda=-\frac{12}{\kappa^{2}}=-\frac{12 N^{2}}{4 \pi^{2}}$. One can treat the next-to-leading term of order $N$ as perturbation of order $N^{2}$ terms. Solving the equation perturbatively, one finds that BH-like metric is modified by

$$
\begin{aligned}
& d s^{2}=g_{\mu \nu} d x^{\mu} d x^{\nu}=-\mathrm{e}^{2 \rho} d t^{2}+\mathrm{e}^{-2 \rho} d r^{2}+r^{2} \sum_{i=1}^{3}\left(d x^{i}\right)^{2} \\
& \mathrm{e}^{2 \rho}=\frac{1}{r^{2}}\left\{-\mu+\left(1+\frac{2}{3} \epsilon\right) r^{4}+2 \epsilon \frac{\mu^{2}}{r^{4}}\right\}, \quad \epsilon \equiv c \kappa^{2}=\frac{1}{16 N} .
\end{aligned}
$$


The radius $r_{h}$ of the horizon and the temperature $T$ are given by

$$
r_{h} \equiv \mu^{\frac{1}{4}}\left(1-\frac{2}{3} \epsilon\right), \quad T=\frac{\mu^{\frac{1}{4}}}{\pi}(1-2 \epsilon)=\frac{\mu^{\frac{1}{4}}}{\pi}\left(1-\frac{1}{8 N}\right) .
$$

It is interesting to calculate the thermodynamical quantities like free energy. After Wick-rotating the time variables by $t \rightarrow i \tau$, the free energy $F$ can be obtained from the action $S$ in (319) where the classical solution is substituted: $F=\frac{1}{T} S$. Using (320) and (321), we find

$$
S=\frac{N^{2} V_{3}}{4 \pi^{2} T} \int_{r_{h}}^{\infty} d r r^{3}\left\{8-\frac{2 \epsilon}{3}\left(40+\frac{72 \mu^{2}}{r^{8}}\right)\right\} .
$$

Here $V_{3}$ is the volume of $3 \mathrm{~d}$ flat space and we assume $\tau$ has a period of $\frac{1}{T}$. The expression of $S$ contains the divergence coming from large $r$. After subtraction of this divergence one gets 235

$$
F=-\frac{N^{2} V_{3} \mu}{4 \pi^{2}}\left(1+\frac{3}{4 N}\right) .
$$

The entropy $\mathcal{S}$ and the mass (energy) $E$ are given by

$$
\mathcal{S}=\frac{N^{2} V_{3} L^{6}(\pi T)^{4}}{\pi^{2} T}\left(1+\frac{3}{4 N}\right), \quad E=\frac{3 N^{2} V_{3} L^{6}(\pi T)^{4}}{4 \pi^{2}}\left(1+\frac{3}{4 N}\right) .
$$

We now compare the above results with those of field theory of $\mathcal{N}=2 S p(N)$ gauge theory. $\mathcal{N}=2$ theory contains $n_{V}=2 N^{2}+N$ vector multiplets and $n_{H}=2 N^{2}+7 N-1$ hypermultiplets. Vector multiplet consists of two Weyl fermions, one complex scalar and one real vector which gives 4 bosonic (fermionic) degrees of freedom on shell and hypermultiplet contains two complex scalars and two Weyl fermions, which also gives 4 bosonic (fermionic) degrees of freedom on shell [248]. Therefore there appear $4 \times\left(n_{V}+n_{H}\right)=16\left(N^{2}+2 N-\frac{1}{4}\right)$ boson-fermion pairs. In the limit which we consider, the interaction between the particles can be neglected. The contribution to the free energy from one boson-fermion pair in the space with the volume $V_{3}$ can be easily estimated [211, 209]. Each pair gives a contribution to the free energy of $\frac{\pi^{2} V_{3} T^{4}}{48}$. Therefore the total free energy $F$ should be 235]

$$
F=-\frac{\pi^{2} V_{3} N^{2} T^{4}}{3}\left(1+\frac{2}{N}-\frac{1}{4 N^{2}}\right)
$$

Comparing (326) with (324), there is the difference of factor $\frac{4}{3}$ in the leading order of $1 / N$ as observed in 211, 209].

Hence, we presented the strong coupling limit of free energy in $\mathcal{N}=2$ SCFT from SG side up to next to leading order term (it was generated by Riemann curvature squared term). Its weak coupling limit (326) obtained from QFT side cannot be presented as strong coupling limit free energy multiplied to some constant. This only holds for leading order terms where mismatch multiplier is $3 / 4$. The next to leading term in (326) should be multiplied to $9 / 32$ in order to produce the corresponding term in (324).

To conclude, in this Chapter, we discussed the role of classical solutions of dilatonic gravity (when dilaton is not constant) in bulk/boundary correspondence for getting strong coupling limit in (super) gauge theory. Of course, we only discussed few topics related with our main purpose: to describe some properties and consequences of (quantum) dilatonic gravity. To understand other topics in this quickly growing field of research one is advised to consult recent review [163]. 


\section{Discussion}

Dilatonic gravity (or scalar-tensor gravity) is the generalization of Einstein theory where scalar partner is added to usual tensor graviton. In this review, we discussed various aspects, methods, results and examples related with dilatonic gravity in quantum regime (or, in classical framework where dual, quantum interpretation may work). The quantum effective action calculated in the models of $2 \mathrm{~d}$ and $4 \mathrm{~d}$ dilatonic gravity (with dilaton coupled matter) helped to give the answers to number of questions. In particular, the renormalizability and quantum equivalence of dilatonic gravities, the existence of fixed points in renormalization group study near two dimensions, quantum corrections to thermodynamics of (dilatonic) black holes and to early Universe evolution have been investigated. In all cases, the corresponding results and examples are given with sufficient details and may be easily understood and generalized for another gravitational backgrounds. The number of interesting phenomena caused by quantum effects like Hawking radiation in dilatonic black holes, anti-evaporation of multiply horizon black holes, inducing of primordial wormholes in Grand Unified Theories and realization of non-singular inflationary Universe are presented.

The study of classical Anti-de Sitter-like solutions of 5d dilatonic gravity (bosonic sector of IIB supergravity) may give the answers to questions in the boundary supersymmetric quantum gauge theory via bulk/boundary correspondence. In this way, dilatonic gravity explains how to find the quark-antiquark potential (with indication to possible confinement), running gauge coupling and free energy (or entropy) of boundary SUSY gauge theory.

We were addressing mainly the aspects of quantum dilatonic gravity. As a result, there are many problems left outside of this review. First of all, we did not discuss the comparison with observational data (macroscopic manifestations of dilaton) indicating sometimes to difficulties related with dilatonic gravity. The reason is very natural: one can always suppose that dilatonic gravity is acceptable only near Planck scale and after that dilaton should quickly decay by some mechanism. From another side, as it was already explained earlier the dilaton may be often regarded as purely technical tool to simplify the consideration (say, 4d Einstein gravity after reduction looks like effective 2d dilatonic gravity, etc.).

Second, while we mentioned that dilaton is unavoidable element of string theory there was no discussion of dilaton from stringy point of view (forgetting the fact that some of the theories under consideration may be string-inspired ones). However, one should note that there may be mechanisms (see, for example, 249]) how to make the presence of even massless dilaton in stringy gravity to be consistent with observational cosmology.

Third, it would be really interesting to develop further the elements of inflationary dilatonic cosmology (fifth chapter) in order to find the deviations from the standard cosmological predictions. One example of this sort comes from the calculation of cosmic background radiation spectrum from the dilatonic theory where dilaton appears as conformal sector of infrared quantum gravity [250].

Fourth, as it follows from fourth chapter the qualitatively new phenomena (like the anti-evaporation of black holes) may be expected in the quantum considerations around (dilatonic) black holes. As so far, the corresponding treatment is semi-classical, the new strong coupling methods to study the gravitational 
physics at extremal conditions should be developed. As it was shown in last chapter one can often realize the classical gravitational systems (like Anti-de Sitter black holes) as quantum non-gravitational systems (supersymmetric gauge theory) using AdS/CFT correspondence. Hence, it is quite possible that right strong coupling methods in quantum gravity may appear from deeper study of above bulk/boundary correspondence. In particular, the hopes are related with the possibility to construct the complete, non-local but finite gravitational effective action (calculated using kind of strong coupling expansion or applicable at extreme conditions). This one and many more similar questions in dilatonic quantum gravity await their final resolution.

\section{Acknoweledgements.}

We would like to thank I. Brevik, I.L. Buchbinder, A.A. Bytsenko, R. Bousso, S.J.Gates, B. Geyer, S.W. Hawking, S. Ichinose, P.M. Lavrov, E. Mottola, I. Oda, O. Obregon, V.V. Obukhov, K.E. Osetrin, A. Sugamoto, A.A. Tseytlin, P. van Nieuwenhuizen and S. Zerbini for useful discussions of related questions. The research by SDO has been supported in part by CONACyT(CP, ref.990356 and grant 28454E) and in part by RFBR.

\section{References}

[1] M.J. Duff, "TWENTY YEARS OF THE WEYL ANOMALY", Classical.Quant.Grav. 11 (1994) 1387, hep-th/9308075.

[2] I.L. Buchbinder, S.D. Odintsov and I.L. Shapiro, EFFECTIVE ACTION IN QUANTUM GRAVITY, IOP publishing, Bristol and Philadelphia, 1992.

[3] J.P. Mbelek and M. Lachize-Rey, "LONG-RANGE ACCELERATION INDUCED BY A SCALAR FIELD EXTERNAL TO GRAVITY AND THE INDICATION FROM PIONER 10/11, GALILEO AND ULYSSES DATA", Phys.Rev. D to appear, gr-qc/9910105.

[4] S.D. Odintsov and I.L. Shapiro, "ONE-LOOP RENORMALIZATION OF TWO-DIMENSIONAL INDUCED QUANTUM GRAVITY”, Phys.Lett. B263 (1991) 183.

[5] S.D. Odintsov and I.L. Shapiro, "PERTURBATIVE FINITENESS OF 2D INDUCED QUANTUM GRAVITY", Mod.Phys.Lett. A7 (1992) 437.

[6] S.D. Odintsov and I.L. Shapiro, "PERTURBATIVE ANALYSIS OF TWO-DIMENSIONAL QUANTUM GRAVITY: RENORMALIZATION, GAUGE DEPENDENCE AND FINITENESS", Int.J.Mod.Phys. D1 (1993) 571.

[7] T. Banks and M. O'Loughlin, "TWO-DIMENSIONAL QUANTUM GRAVITY IN MINKOWSKI SPACE", Nucl.Phys. B362 (1991) 649.

[8] R. Jackiw, "LOWER DIMENSIONAL GRAVITY", Nucl.Phys. B252 (1985) 343. 
[9] C. Teitelboim, "REMARKS ON THE QUANTUM MECHANICS OF THE RELATIVISTIC STRING", Phys.Lett. 126B (1983) 41.

[10] J.G. Russo and A. Tseytlin, "SCALAR-TENSOR QUANTUM GRAVITY IN TWO DIMENSIONS", Nucl.Phys. B382 (1992) 259, hep-th/9201021.

[11] R. Kantowski and C. Marzban, "ONE-LOOP VILKOVISKY-DE WITT COUNTERTERMS FOR 2D GRAVITY PLUS SCALAR FIELD THEORY”, Phys.Rev. D46 (1992) 5449.

[12] F.D. Mazzitelli and N. Mohammedi, "CLASSICAL GRAVITY COUPLED TO LIOUVILLE THEORY", Nucl.Phys. B401 (1993) 239.

[13] S. Ichinose, "PERTURBATIVE APPROACH TO TWO-DIMENSIONAL QUANTUM GRAVITY", Int.J.Mod.Phys. A8 (1993) 2735.

[14] E. Elizalde, S. Naftulin and S.D. Odintsov, "COVARIANT EFFECTIVE ACTION AND ONELOOP RENORMALIZATION OF 2D DILATON GRAVITY WITH FERMIONIC MATTER", Phys.Rev. D49 (1994) 2852.

[15] G. 't Hooft and M. Veltman, "ONE-LOOP DIVERGENCES IN THE THEORY OF GRAVITATION", Ann.Inst.H.Poincare 20 (1974) 69.

[16] E. Elizalde and S.D. Odintsov, "ONE-LOOP RENORMALIZATION IN TWO-DIMENSIONAL MATTER- DILATON QUANTUM GRAVITY AND CHARGED BLACK HOLES" Nucl.Phys. B399 (1993) 581.

[17] E. Elizalde, S. Naftulin and S.D. Odintsov, "CONVENIENT VERSUS UNIQUE EFFECTIVE ACTION FORMALISM IN 2D DILATON-MAXWELL QUANTUM GRAVITY", Z.Phys. C60 (1993) 327.

[18] E. Elizalde, S. Naftulin and S.D. Odintsov, "THE RENORMALIZATION STRUCTURE AND QUANTUM EQUIVALENCE OF 2D DILATON GRAVITIES", Int.J.Mod.Phys. A9 (1994) 933.

[19] S. Weinberg, "ULTRAVIOLET DIVERGENCES IN QUANTUM THEORIES OF GRAVITATION", in General Relativity, an Einstein Centenary Survey, eds. S.W. Hawking and W. Israel (Cambridge Univ. Press, Cambridge, 1979).

[20] R. Gastmans, R. Kallosh and C. Truffin, "QUANTUM GRAVITY NEAR TWO DIMENSIONS", Nucl.Phys. B133 (1978) 417.

[21] S.M. Christensen and M.J. Duff, "QUANTUM GRAVITY IN TWO + EPSILON DIMENSIONS", Phys. Lett. 79B (1978) 213.

[22] H. Kawai and M. Ninomiya, "RENORMALIZATION GROUP AND QUANTUM GRAVITY", Nucl.Phys. B336 (1990) 115. 
[23] J. Nishimura, S. Tamura and A. Tsuchiya, "R**2 GRAVITY IN (2+EPSILON)-DIMENSIONAL QUANTUM GRAVITY", Mod.Phys.Lett. A9 (1994) 3565, hep-th/9405059.

[24] I. Jack and D.R.T. Jones, "THE EPSILON EXPANSION OF TWO-DIMENSIONAL QUANTUM GRAVITY", Nucl.Phys. B358 (1991) 695.

[25] J. Ambjørn and J. Jurkiewicz, "FOUR-DIMENSIONAL SIMPLICIAL QUANTUM GRAVITY", Phys.Lett. B278 (1992) 42.

[26] I. Antoniadis, P.O. Mazur and E. Mottola, "SCALING BEHAVIOR OF QUANTUM FOUR - GEOMETRIES", Phys. Lett. B323 (1994) 284, hep-th/9301002.

[27] J. Ambjørn, S. Jain, J. Jurkiewicz and C.F. Kristjansen, "OBSERVING 4D BABY UNIVERSES IN QUANTUM GRAVITY", Phys.Lett. B305 (1993) 208, hep-th/9303041.

[28] S. Kojima, N. Sakai and Y. Tanii, "DILATON GRAVITY IN (2+EPSILON)-DIMENSIONS", Nucl.Phys. B426 (1994) 223, hep-th/9405072.

[29] T. Aida, Y. Kitazawa, H. Kawai and M. Ninomiya, "CONFORMAL INVARIANCE AND RENORMALIZATION GROUP IN QUANTUM GRAVITY NEAR TWO-DIMENSIONS", Nucl.Phys. B427 (1994) 158, hep-th/9404171.

[30] E. Elizalde and S.D. Odintsov, "DILATON-MAXWELL GRAVITY WITH MATTER NEAR TWO DIMENSIONS", Fortschr.Phys. 44 (1996) 85, hep-th/9501015.

[31] E. Elizalde and S.D. Odintsov, "DILATONIC GRAVITY NEAR TWO DIMENSIONS AND ASYMPTOTIC FREEDOM OF THE GRAVITATIONAL COUPLING CONSTANT", Phys.Lett. B347 (1995) 211, hep-th/9501067.

[32] K.G. Wilson and J. Kogut, "THE RENORMALIZATION GROUP AND THE EPSILON EXPANSION", Phys.Rep. 12 (1974) 75.

[33] J. Zinn-Justin, 'QUANTUM FIELD THEORY AND CRITICAL PHENOMENA, (Oxford Univ. Press, Oxford, 1989).

[34] E. Brezin, J.C. Le Guillou and J. Zinn-Justin, "FIELD THEORETICAL APPROACH TO CRITICAL PHENOMENA", in Phase transitions and critical phenomena, Vol. 6, eds. C. Domb and M. Green (Academic Press, London, 1976).

[35] C. Callan, S.B. Giddings, J. Harvey and A. Strominger, "EVANESCENT BLACK HOLES", Phys.Rev. D45 (1992) R1005, hep-th/9111056.

[36] M. McGuigan, C. Nappi and S.A. Yost, "CHARGED BLACK HOLES IN TWO-DIMENSIONAL STRING THEORY", Nucl.Phys. B375 (1992) 421, hep-th/9111038.

[37] O. Lechtenfeld and C. Nappi, "DILATON GRAVITY AND NO HAIR THEOREM IN TWODIMENSIONS", Phys.Lett. B288 (1992) 72, hep-th/9204026. 
[38] D. Louis-Martinez and G. Kunstatter, "TWO-DIMENSIONAL DILATON GRAVITY COUPLED TO ABELIAN GAUGE FIELD", Phys.Rev. D52 (1995) 3494.

[39] G.W. Gibbons, "ANTIGRAVITATING BLACK HOLE SOLITONS WITH SCALAR HAIR IN N $=4$ SUPERGRAVITY", Nucl.Phys. B207 (1982) 337.

[40] G.W. Gibbons and K. Maeda, "BLACK HOLES AND MEMBRANES IN HIGHER DIMENSIONAL THEORIES WITH DILATON FIELDS", Nucl.Phys. B298 (1988) 74.

[41] S. Kojima, N. Sakai and Y. Tanii, "SUPERGRAVITY IN (2+EPSILON)-DIMENSIONS", Int.J. Mod.Phys. A9 (1994) 5415, hep-th/9311045.

[42] E. Elizalde, A.G. Jacksenaev, S.D. Odintsov and I.L. Shapiro, "A FOUR-DIMENSIONAL THEORY FOR QUANTUM GRAVITY WITH CONFORMAL AND NON-CONFORMAL EXPLICIT SOLUTIONS", Class.Quantum Grav. 12 (1995) 1385, hep-th/9412061

[43] I. Antoniadis and E. Mottola, "4D QUANTUM GRAVITY IN THE CONFORMAL SECTOR", Phys.Rev. D45 (1992) 2013.

[44] S.D. Odintsov, "CURVED SPACE-TIME FORMULATION OF THE CONFORMAL SECTOR FOR 4D QUANTUM GRAVITY", Z.Phys. C54 (1992) 531.

[45] K. Hamada, "A CANDIDATE FOR RENORMALIZABLE AND DIFFEOMOROPHISM INVARIANT 4D QUANTUM THEORY OF GRAVITY", hep-th/9912098.

[46] S. Nojiri and S.D. Odintsov, "TRACE ANOMALY AND NON-LOCAL EFFECTIVE ACTION FOR 2D CONFORMALLY INVARIANT SCALAR INTERACTING WITH DILATON", Mod.Phys.Lett. A12 (1997) 2083, hep-th/9706009.

[47] S. Nojiri and S.D. Odintsov, "TRACE ANOMALY INDUCED EFFECTIVE ACTION FOR 2D AND 4D DILATON COUPLED SCALARS", Phys.Rev. D57 (1998) 2363, hep-th/9706143.

[48] N. Birrell and P. Davies, "QUANTUM FIELDS IN CURVED SPACE", Cambridge University Press, 1982.

[49] S. Ichinose and N. Ikeda, "NEW FORMULATION OF ANOMALY, ANOMALY FORMULA AND GRAPHICAL REPRESENTATION", Phys.Rev. D53 (1996) 5932, hep-th/9509073.

[50] R. Bousso and S.W. Hawking, "TRACE ANOMALY OF DILATON COUPLED SCALARS IN TWO-DIMENSIONS", Phys.Rev. D56 (1997) 7788, hep-th/9705236.

[51] T. Chiba and M. Siino, "DISAPPEARANCE OF BLACK HOLE CRITICALITY IN SEMICLASSICAL GENERAL RELATIVITY", Mod.Phys.Lett. A12 (1997) 709.

[52] S. Ichinose, "WEYL ANOMALY OF 2D DILATON - SCALAR GRAVITY AND HERMITICITY OF SYSTEM OPERATOR", Phys.Rev. D57 (1998) 6224, hep-th/9707025. 
[53] A. Mikovic and V. Radovanovic, "ONE-LOOP EFFECTIVE ACTION FOR SPHERICAL SCALAR FIELD COLLAPSE", Class.Quant.Grav. 15 (1998) 827, hep-th/9706066.

[54] W. Kummer, H.Liebl and D.V. Vassilevich, "HAWKING RADIATION FOR NONMINIMALLY COUPLED MATTER FROM GENERALIZED 2D BLACK HOLE MODELS", Mod.Phys.Lett. A12 (1997) 2683, hep-th/9707041.

[55] J.S. Dowker, "CONFORMAL ANOMALY IN 2D DILATON SCALAR THEORY", Class.Quant.Grav. 15 (1998) 1881, hep-th/9802029.

[56] S. Ichinose and S.D. Odintsov, "CONFORMAL ANOMALY IN 4D GRAVITY-MATTER THEORIES NONMINIMALLY COUPLED WITH DILATON", Nucl.Phys. B539 (1999) 643, hepth/9802043.

[57] S. Nojiri and S.D. Odintsov, "QUANTUM (IN)STABILITY OF 2D CHARGED DILATON BLACK HOLES AND 3D ROTATING BLACK HOLES", Phys.Rev. D59 (1999) 044003, hep-th/9806055.

[58] Yu. V. Gusev and A.I. Zelnikov, "TWO-DIMENSIONAL EFFECTIVE ACTION FOR MATTER FIELDS COUPLED TO THE DILATON", hep-th/9910198.

[59] P. van Nieuwenhuizen, S. Nojiri and S.D. Odintsov, "CONFORMAL ANOMALY FOR 2D AND 4D DILATON COUPLED SPINORS", Phys.Rev. D60 (1999) 08014, hep-th/9901119.

[60] S. Deser and A. Schwimmer, "GEOMETRIC CLASSIFICATION OF CONFORMAL ANOMALIES IN ARBITRARY DIMENSIONS", Phys.Lett. B309 (1993) 279, hep-th/9302047.

[61] R.J. Riegert, "A NONLOCAL ACTION FOR THE TRACE ANOMALY", Phys.Lett. B134 (1984)56.

[62] D. Anselmi, ANOMAliES, UNITARITY AND QUANTUM IRREVERSIBILITY, Ann.Phys. 276(1999)361; hep-th/9903059.

[63] E.S. Fradkin and A. Tseytlin, "CONFORMAL ANOMALY IN WEYL THEORY AND ANOMALY FREE SUPERCONFORMAL THEORIES", Phys.Lett. 134B (1984) 187.

[64] I.L. Buchbinder, S.D. Odintsov and I.L. Shapiro, "NON-SINGULAR COSMOLOGICAL MODEL WITH TORSION INDUCED BY VACUUM QUANTUM EFFECTS", Phys.Lett. B162 (1985) 92

[65] T. Eguchi and P. Freund, "QUANTUM GRAVITY AND WORLD TOPOLOGY", Phys.Rev.Lett. 37 (1976) 1251.

[66] P. van Nieuwenhuizen, "SUPERGRAVITY", Phys.Rept. 68 (1981) 189.

[67] R. Endo and M. Takao, "PATH INTEGRAL DERIVATION OF THE CHIRAL ANOMALIES IN HIGHER DIMENSIONAL CURVED SPACE-TIME", Prog.Theor.Phys. 73 (1985) 803.

[68] S. Nojiri and S.D. Odintsov, "CONFORMAL ANOMALY FOR DILATON COUPLED ELECTROMAGNETIC FIELD", Phys.Lett. B426 (1998) 27, hep-th/9801052. 
[69] L.S. Brown and J.P. Cassidy, "STRESS TENSORS AND THEIR TRACE ANOMALIES IN CONFORMALLY FLAT SPACE-TIMES", Phys.Rev. D16 (1977) 1712.

[70] S. Christensen and M. Duff, "NEW GRAVITATIONAL INDEX THEOREMS AND SUPERTHEOREMS", Nucl.Phys. B154 (1979) 301.

[71] B. Geyer and P.M. Lavrov, COVARIANT QUANTIZATION OF GAUGE THEORIES, Lectures given by P.M. Lavrov at Leipzig University, 1998.

[72] S. Nojiri and S.D. Odintsov, "TRACE ANOMALY INDUCED EFFECTIVE ACTION AND 2D BLACK HOLES FOR DILATON COUPLED SUPERSYMMETRIC THEORIES", Phys.Rev. D57 (1998) 4847, hep-th/9801180.

[73] K. Higashijima, T. Uematsu and Y.Z. Yu, "DYNAMICAL SUPERSYMMETRY BREAKING IN TWO-DIMENSIONAL N=1 SUPERGRAVITY THEORIES", Phys.Lett. 139B (1984) 161.

[74] T. Uematsu, "STRUCTURE OF N=1 CONFORMAL AND POINCARE SUPERGRAVITY IN (1+1)-DIMENSIONS AND (2+1)-DIMENSIONS", Z.Phys. C29 (1985) 143.

[75] T. Uematsu, "CONSTRAINTS AND ACTIONS IN TWO-DIMENSIONAL AND THREEDIMENSIONAL N=1 CONFORMAL SUPERGRAVITY", Z.Phys. C32 (1986) 33.

[76] S.J. Gates, M.T. Grisaru, M. Rocek and W. Siegel, SUPERSPACE OR ONE THOUSAND AND ONE LESSONS IN SUPERSYMMETRY, Benjamin/Cummings (1983) (Frontiers in Physics, 58).

[77] S.J. Gates, T. Kadoyoshi, S. Nojiri and S.D. Odintsov, "QUANTUM COSMOLOGY IN THE MODELS OF 2D AND 4D DILATONIC SUPERGRAVITY WITH WZ MATTER", Phys.Rev. D58 (1998) 084026, hep-th/9802139.

[78] S.J. Gates, S. Gukov and E. Witten, "TWO TWO-DIMENSIONAL SUPERGRAVITY THEORIES FROM CALABI-YAU FOUR-FOLDS", hep-th/0005120.

[79] S.W. Hawking, "PARTICLE CREATION BY BLACK HOLES" Comm.Math.Phys. 43 (1975) 199.

[80] G. 't Hooft, "THE BLACK HOLE INTERPRETATION OF STRING THEORY" Nucl.Phys. B335 (1990) 138.

[81] Y. Aharonov, A. Casher and S. Nissinov, "THE UNITARITY PUZZLE AND PLANCK MASS STABLE PARTICLES" Phys.Lett. 191B (1987) 51.

[82] C.F.E. Holzhey and F. Wilczek, "BLACK HOLES AS ELEMENTARY PARTICLES", Nucl.Phys. B380 (1992) 447, hep-th/9202014.

[83] J.G. Russo, L. Susskind and L. Thorlacius, "THE ENDPOINT OF HAWKING RADIATION", Phys.Rev. D46 (1992) 3444, hep-th/9206070.

[84] S.P. de Alwis, "QUANTIZATION OF A THEORY OF 2-D DILATON GRAVITY", Phys.Lett. B289 (1992) 278, hep-th/9205069. 
[85] A. Bilal and C. Callan, "LIOUVILLE MODELS OF BLACK HOLE EVAPORATION", Nucl.Phys. B394 (1993) 73, hep-th/9205089.

[86] R.B. Mann, "CONSERVATION LAWS AND 2-D BLACK HOLES IN DILATON GRAVITY", Phys.Rev. D47 (1993) 4438, hep-th/9206044.

[87] J. Polchinski and A. Strominger, "A POSSIBLE RESOLUTION OF THE BLACK HOLE INFORMATION PUZZLE", Phys.Rev. D50 (1994) 7403, hep-th/9407008.

[88] T. Klobsch and T. Strobl, "CLASSICAL AND QUANTUM GRAVITY IN (1+1)-DIMENSIONS. PART 2: THE UNIVERSAL COVERINGS", Class.Quant.Grav. 13 (1996) 965, gr-qc/9511081.

[89] S. Bose, L. Parker and Y. Peleg, "SEMIINFInITE THROAT AS THE END STATE GEOMETRY OF TWO-DIMENSIONAL BLACK HOLE EVAPORATION" Phys.Rev. D52 (1995) 3512, hepth/9502098.

[90] J. Cruz, A. Fabbri, D.J. Navarro and J. Navarro-Salas, "INTEGRABLE MODELS AND DEGENERATE HORIZONS IN TWO-DIMENSIONAL GRAVITY", Phys.Rev. D61 (2000) 024011, hepth/9906187.

[91] A. Strominger, LES HOUCHES LECTURES ON BLACK HOLES, hep-th/9501071.

[92] S. Nojiri and I. Oda, "SEMICLASSICAL APPROACH TO CHARGED DILATONIC BLACK HOLE IN TWO DIMENSIONS", Nucl.Phys. B406 (1993) 499, hep-th/9209118.

[93] W. Kummer and D.V. Vassilevich, "HAWKING RADIATION FROM DILATON GRAVITY IN 1+1 DIMENSIONS: A PEDAGOGICAL REVIEW", Annalen Phys. 8 (1999) 801, gr-qc/9907041.

[94] S. Nojiri and I. Oda, "DILATONIC SUPERGRAVITY IN TWO-DIMENSIONS AND THE DISAPPEARANCE OF QUANTUM BLACK HOLE", Mod.Phys.Lett. A8 (1993) 53.

[95] S.W. Hawking, "EVAPORATION OF TWO-DIMENSIONAL BLACK HOLES", Phys.Rev.Lett. 69 (1992) 406, hep-th/9203052.

[96] T. Banks, A. Dabholkar, M.R. Douglas and M. O'Loughlin, "ARE HORNED PARTICLES THE CLIMAX OF HAWKING EVAPORATION?", Phys.Rev. D45 (1992) 3607, hep-th/9201061.

[97] N. Ishibashi, M. Li and R. Steif, "TWO-DIMENSIONAL CHARGED BLACK HOLES IN STRING THEORY", Phys.Rev.Lett. 67 (1991) 3336.

[98] S. Coleman, J. Preskill and F. Wilczek, "QUANTUM HAIR ON BLACK HOLES”, Nucl.Phys. B378 (1992) 175-246, hep-th/9201059.

[99] J.G. Russo, L. Susskind and L. Thorlacius, "BLACK HOLE EVAPORATION IN (1+1)DIMENSIONS", Phys. Lett. B292 (1992) 13, hep-th/9201074.

[100] M. Alves, "HAWKING RADIATION IN THE DILATON GRAVITY WITH A NON-MINIMALLY COUPLED SCALAR FIELD”, Int.J.Mod.Phys. D8 (1999) 687, hep-th/9909138. 
[101] J.M. Bardeen, B. Carter and S.W. Hawking, "THE FOUR LAWS OF BLACK HOLE MECHANICS", Comm.Math.Phys. 31 (1973) 161.

[102] S.W. Hawking, HAWKING ON THE BIG BANG AND BLACK HOLES, World Sci., Singapore, 1993.

[103] J.D. Bekenstein, "BLACK HOLES AND THE SECOND LAW", Nuovo Cim.Lett. 4 (1972) 737.

[104] A. Strominger and C. Vafa, "MICROSCOPIC ORIGIN OF THE BEKENSTEIN-HAWKING ENTROPY", Phys.Lett. B379 (1996) 99, hep-th/9601029.

[105] G.T. Horowitz and A. Strominger, "COUNTING STATES OF NEAR EXTREMAL BLACK HOLES", Phys.Rev.Lett. 77 (1996) 2368, hep-th/9602051.

[106] G.T. Horowitz, J.M. Maldacena and A. Strominger, "NONEXTREMAL BLACK HOLE MICROSTATES AND U DUALITY", Phys.Lett. B383 (1996) 151, hep-th/9603109.

[107] G.T. Horowitz, D.A. Lowe and J.M. Maldacena, "STATISTICAL ENTROPY OF NONEXTREMAL FOUR-DIMENSIONAL BLACK HOLES AND U DUALITY", Phys.Rev.Lett. 77 (1996) 430, hep-th/9603195.

[108] A. Peet, "THE BEKENSTEIN FORMUlA AND STRING THEORY (N-BRANE THEORY).", Class.Quant.Grav. 15 (1998) 3291, hep-th/9712253.

[109] S. Carlip, "WHAT WE DON'T KNOW ABOUT BTZ BLACK HOLE ENTROPY?", Class.Quant.Grav. 15 (1998) 3609, hep-th/9806026.

[110] A. Bytsenko, L. Vanzo and S. Zerbini, "QUANTUM CORRECTION TO THE ENTROPY OF THE (2+1)-DIMENSIONAL BLACK HOLE", Phys.Rev. D57 (1998) 4917, gr-qc/9710106.

[111] H. Saida and J. Soda, "STATISTICAL ENTROPY OF BTZ BLACK HOLE IN HIGHER CURVATURE GRAVITY", Phys.Lett. B471 (2000) 358, gr-qc/9909061.

[112] T. Jacobson, "BLACK HOLE ENTROPY AND INDUCED GRAVITY", gr-qc/9404039.

[113] S. Nojiri and S.D. Odintsov, "THERMODYNAMICS OF SCHWARZSCHILD-(ANTI-)DE SITTER BLACK HOLES WITH ACCOUNT OF QUANTUM CORRECTIONS", Int.J.Mod.Phys. A15 (2000) 989, hep-th/9905089.

[114] R. Bousso and S.W. Hawking, "(ANTI)EVAPORATION OF SCHWARZSCHILD-DE SITTER BLACK HOLES", Phys.Rev. D57 (1998) 2436, hep-th/9709224.

[115] R. Bousso, "PROLIFERATIOn OF DE SITTER SPACE", Phys.Rev. D58 (1998) 083511, hepth/9805081.

[116] M. Burić, V. Radovanović and A. Miković, "ONE-LOOP CORRECTION FOR SCHWARZSCHILD BLACK HOLE VIA 2D DILATON GRAVITY", Phys.Rev. D59 (1999) 084002, gr-qc/9804083. 
[117] F. Lombardo, F.D. Mazzitelli and J. Russo, "ENERGY-MOMENTUM TENSOR FOR SCALAR FIELDS COUPLED TO THE DILATON IN TWO DIMENSIONS", Phys. Rev. D59 (1999) 084002, gr-qc/9808048.

[118] R. Balbinot and A. Fabbri, "HAWKING RADIATION BY EFFECTIVE TWO-DIMENSIONAL THEORIES", Phys.Rev. D59 (1999) 044031, hep-th/9807123.

[119] A.J.M. Medved and G. Kunstatter, "QUANTUM CORRECTIONS TO THE THERMODYNAMICS OF CHARGED 2D BLACK HOLES", Phys.Rev. D60 (1999) 104029, hep-th/9904070.

[120] W.T. Kim and J. Oh, "DILATON DRIVEN HAWKING RADIATION IN ADS(2) BLACK HOLE", Phys.Lett. B461 (1999) 189, hep-th/9905007.

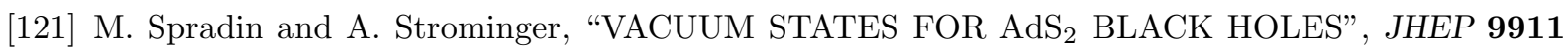
(1999) 021, hep-th/9904143.

[122] G. Gibbons, "TUNNELING WITH A NEGATIVE COSMOlOGICAL CONSTANT", Nucl.Phys. B472 (1996) 683, hep-th/9601075.

[123] G. Gibbons, "THE ENTROPY AND STABILITY OF THE UNIVERSE”, Nucl.Phys. B292 (1987) 784.

[124] G. Gibbons, 'SOBOLEV'S INEQUALITY, JENSEN'S THEOREM AND THE MASS AND ENTROPY OF THE UNIVERSE", Nucl.Phys. B310 (1988) 636.

[125] M. Buric and V. Radovanovic "QUANTUM CORRECTIONS FOR THE REISSNERNORDSTROM BLACK HOLE", Class.Quant.Grav. 16 (1999) 3937, gr-qc/9907036.

[126] H. Nariai, Sci.Rep.Tohoku Univ.Ser.I 35 (1951) 62.

[127] J.B. Hartle and S.W. Hawking, "WAVE FUNCTION OF THE UNIVERSE", Phys.Rev. D28 (1983) 2960 .

[128] R. Bousso and S.W. Hawking, "PAIR CREATION OF BLACK HOLES DURING INFLATION", Phys.Rev. D54 (1996) 6312, gr-qc/9606052.

[129] A. Bytsenko, S. Nojiri and S.D. Odintsov, "QUANTUM GENERATION OF SCHWARZSCHILDDE SITTER (NARIAI) BLACK HOLES IN EFFECTIVE DILATON - MAXWELL GRAVITY", Phys.Lett. B443 (1998) 121, hep-th/9808109.

[130] S. Nojiri and S.D. Odintsov, "QUANTUM EVOLUTION OF SCHWARZSCHILD-DE SITTER (NARIAI) BLACK HOLES", Phys.Rev. D59 (1999) 044026, hep-th/9804033.

[131] E. Elizalde, S. Nojiri and S.D. Odintsov, "POSSIBLE QUANTUM INSTABILITY OF PRIMORDIAL BLACK HOLES", Phys.Rev. D59 (1999) 061501, hep-th/9901026.

[132] G.W. Gibbons and M.J. Perry, "THE PHYSICS OF 2D STRINGY SPACE-TIMES", Int.J.Mod.Phys. D1 (1992) 335, hep-th/9204090. 
[133] M. Bañados, C. Teitelboim and J. Zanelli, "THE BLACK HOLE IN THREE-DIMENSIONAL SPACE-TIME", Phys.Rev.Lett. 69 (1992) 1849, hep-th/9204099.

[134] V. Frolov, P. Sutton and A. Zelnikov, THE DIMENSIONAL-REDUCTION ANOMALY, Phys.Rev. D61 (2000) 024021, hep-th/9909086.

[135] S. Nojiri and S.D. Odintsov, ANOMALY INDUCED EFFECTIVE ACTIONS AND RELIABILITY OF S-WAVE APPROXIMATION, Phys.Lett. B463 (1999) 57, hep-th/9904148

[136] P. Hajicek, "SPHERICALLY SYMMETRIC SYSTEMS OF FIELDS AND BLACK HOLES. 2. APPARENT HORIZON IN CANONICAL FORMALISM", Phys.Rev. D30 (1984) 1178.

[137] P. Thomi, B. Isaak and P. Hajicek, "SPHERICALLY SYMMETRIC SYSTEMS OF FIELDS AND BLACK HOLES. 1. APPARENT HORIZON IN CANONICAL FORMALISM", Phys.Rev. D30 (1984) 1168

[138] R. Kantowski and R. Sachs, J.Math.Phys. 7 (1966) 443.

[139] S. Nojiri, O. Obregon, S.D. Odintsov and K.E. Osetrin, "(NON)SINGULAR KANTOWSKI-SACHS UNIVERSE FROM QUANTUM SPHERICALLY REDUCED MATTER", Phys.Rev. D60 (1999) 024008, hep-th/9902035.

[140] F. Mazzitelli and J. Russo, "DILATON QUANTUM COSMOLOGY IN TWO-DIMENSIONS", Phys.Rev. D47 (1993) 4490, hep-th/9211095.

[141] A. Fabbri and J. Russo, "SOluble MODELS IN 2D DILATON GRAVITY", Phys.Rev. D53 (1996) 6995, hep-th/9510109.

[142] M. Gasperini and G. Veneziano, "DILATON PRODUCTION IN STRING COSMOLOGY", Phys.Rev. D50 (1994) 2519, gr-qc/9403031.

[143] W.T. Kim and M.S. Yoon, "INITIAL SINGULARITY FREE QUANTUM COSMOLOGY IN TWO-DIMENSIONAL BRANS-DICKE THEORY", Phys.Rev. D58 (1998) 084014, hepth/9803081.

[144] O. Obregon and M. Ryan, Jr., "QUANTUM PLANCK SIZE BLACK HOLE STATES WITHOUT A HORIZON", Mod.Phys.Lett. A13 (1998) 3251.

[145] B. Geyer, S.D. Odintsov and S. Zerbini, "INFLATIONARY BRANS-DICKE QUANTUM UNIVERSE", Phys.Lett. B460 (1999) 58, gr-qc/9905073.

[146] C.M. Will, THEORY AND EXPERIMENTS IN GRAVITATIONAL PHYSICS , Cambridge University Press, Cambridge (1993).

[147] J. Polchinski, STRING THEORY, Cambridge University Press, Cambridge (1998). 
[148] V. Faraoni, E Gunzig and P. Nardone, "CONFORMAL TRANSFORMATIONS IN CLASSICAL GRAVITATIONAL THEORIES AND IN COSMOLOGY", Fundamentals of Cosmic Physics 20 (1999) 121, gr-qc/9811047.

[149] A. Bytsenko, G. Cognola, L. Vanzo, and S. Zerbini, "QUANTUM FIELDS AND EXTENDED OBJECTS IN SPACE-TIMES WITH CONSTANT CURVATURE SPATIAL SECTION", Phys. Repts. 266 (1996) 1, hep-th/9505061.

[150] A. Starobinsky, A NEW TYPE OF ISOTROPIC COSMOLOGICAL MODELS WITHOUT SINGULARITY, Phys.Lett. B91 (1980) 99.

[151] S.G. Mamaev and V.M. Mostepanenko, ISOTROPIC COSMOLOGICAL MODELS DEFINED BY QUANTUM EFFECTS, JETP 78 (1980) 20.

[152] M. Kaku, P.K. Townsend and P. van Nieuwenhuizen, " PROPERTIES OF CONFORMAL SUPERGRAVITY", Phys.Rev. D 17, 3179 (1978).

[153] E. Bergshoeff, M. de Roo and B. de Wit, "EXTENDED CONFORMAL SUPERGRAVITY", Nucl. Phys. B 182, 173 (1981).

[154] E.S. Fradkin and A.A. Tseytlin, CONFORMAL SUPERGRAVITY, Phys.Repts. 119 (1985) 233.

[155] H. Liu and A.A. Tseytlin, "D = 4 SUPER YANG-MILLS, D = 5 GAUGED SUPERGRAVITY, AND D = 4 CONFORMAL SUPERGRAVITY", Nucl.Phys. B533 (1998) 88, hep-th/9804083.

[156] M.S. Morris and K.S. Thorne, "WORMHOLES IN SPACE-TIME AND THEIR USE FOR INTERSTELLER TRAVEL: A TOOL FOR TEACHING GENERAL RELATIVITY”, Am.J.Phys. 56 (1988) 395.

[157] M.S. Thorne, K.S. Thorne and U. Yurtsever, "WORMHOLES, TIME MACHINES, AND THE WEAK ENERGY CONDITION", Phys.Rev.Lett. 61 (1988) 1446.

[158] G. Gibbons, "WORMHOLES ON THE WORLD VOLUME: BORN-INFELD PARTICLES AND DIRICHLET P-BRANE", hep-th/9801106.

[159] D. Hochberg, A. Popov and S.N. Sushkov, "SELFCONSISTENT WORMHOLE SOLUTIONS OF SEMICLASSICAL GRAVITY" Phys.Rev.Lett. 78 (1997) 2050, gr-qc/9701064.

[160] V.

Khatsymovsky, TOWARDS POSSIBILITY OF SELF-MAINTAINED VACUUM TRAVERSIBLE WORMHOLE, In proceedings of II Int.Conf.Quantum Field Theory and Gravity, Eds.I.L. Buchbinder and K.E. Osetrin, TGPU Publishing, Tomsk, 1997.

[161] S. Nojiri, O. Obregon, S.D. Odintsov and O. Osetrin, INDUCED WORMHOLES DUE TO QUANTUM EFFECTS OF SPHERICALLY REDUCED MATTER IN LARGE N APPROXIMATION, Phys.Lett. B449 (1999) 173, hep-th/9812164. 
[162] S. Nojiri, O. Obregon, S.D. Odintsov and K.E. Osetrin, "CAN PRIMORDIAL WORMHOLES BE INDUCED BY GUTS AT THE EARLY UNIVERSE?", Phys.Lett. B458 (1999) 19, gr-qc/9904035.

[163] O. Aharony, S. Gubser, J. Maldacena, H. Ooguri and Y. Oz, "LARGE N FIELD THEORIES, STRING THEORY AND GRAVITY", Phys.Repts. 323 (2000) 183, hep-th/9905111.

[164] J. Maldacena, "THE LARGE N LIMIT OF SUPERCONFORMAL FIELD THEORIES AND SUPERGRAVITY", Adv.Theor.Math.Phys. 2 (1998) 231, hep-th/9711200.

[165] E. Witten, "ANTI-DE SITTER SPACE AND HOLOGRAPHY", Adv.Theor.Math.Phys. 2 (1998) 253 , hep-th/9802150.

[166] M. Henningson and K. Skenderis, "THE HOLOGRAPHIC WEYL ANOMALY", JHEP 9807023 (1998), hep-th/9806087.

[167] S. Nojiri and S.D. Odintsov, "CONFORMAL ANOMALY FOR DILATON COUPLED THEORIES FROM ADS / CFT CORRESPONDENCE”, Phys.Lett. B444 (1998) 92, hep-th/9810008.

[168] G.W. Gibbons and S.W. Hawking, "ACTION INTEGRALS AND PARTITION FUNCTIONS IN QUANTUM GRAVITY", Phys.Rev. D15 (1977) 2752.

[169] C. Fefferman and C.R. Graham, "CONFORMAL INVARIANTS", in Elie Cartan et les Math. d'aujourdhui, Asterisque, 1985.

[170] J.D. Brown and M. Henneaux, "CENTRAL CHARGES IN THE CANONICAL REALIZATION OF ASYMPTOTIC SYMMETRIES: AN EXAMPLE FROM THREE-DIMENSIONAL GRAVITY", Comm.Math.Phys. 104 (1986) 207.

[171] M. Blau, K.S. Narain and E. Gava, "ON SUBLEADING CONTRIBUTIONS TO THE ADS / CFT TRACE ANOMALY", JHEP 9909 (1999) 018, hep-th/9904179.

[172] S. Nojiri and S.D. Odintsov, "ON THE CONFORMAL ANOMALY FROM HIGHER DERIVATIVE GRAVITY IN ADS / CFT CORRESPONDENCE”, Int.J.Mod.Phys. A15 (2000) 413, hepth/9903033.

[173] S. Nojiri, S.D. Odintsov, S. Ogushi, A. Sugamoto, and M. Yamamoto, "AXION-DILATONIC CONFORMAL ANOMALY FROM AdS/CFT CORRESPONDENCE", Phys.Lett. B465 (1999) 128, hep-th/9908066.

[174] G.W. Gibbons, M.B. Green and M.J. Perry, "INSTANTONS AND SEVEN-BRANES IN TYPE IIB SUPERSTRING THEORY", Phys.Lett. B370 (1996) 37, hep-th/9511080.

[175] C. Imbimbo, A.Schwimmer, S. Theisen and S. Yankielowicz, "DIFFEOMORPHISMS AND HOLOGRAFIC ANOMALY", Class.Quant.Grav. 17 (2000) 1129, hep-th/9910267.

[176] S. Gubser, I. Klebanov and A. Polyakov, "GAUGE THEORY CORRELATORS FROM NONCRITICAL STRING THEORY", Phys.Lett. B428 105, hep-th/9802109. 
[177] I.R. Klebanov and A.A. Tseytlin, "D-BRANES AND DUAL GAUGE THEORIES IN TYPE 0 STRINGS", Nucl.Phys. B546 (1999) 155, hep-th/9811035.

[178] I.R. Klebanov and A.A. Tseytlin, "ASYMPTOTIC FREEDOM AND INFRARED BEHAVIOR IN THE TYPE 0 STRING APPROACH TO GAUGE THEORY", Nucl.Phys. B548 (1999) 231, hep-th/9812089.

[179] I.R. Klebanov and A.A. Tseytlin, "A NONSUPERSYMMETRIC LARGE N CFT FROM TYPE 0 STRING THEORY”, JHEP 03 (1999) 015, hep-th/9901101.

[180] J.A. Minahan, "GLUEBALL MASS SPECTRA AND OTHER ISSUES FOR SUPERGRAVITY DUALS OF QCD MODELS", JHEP 01 (1999) 020, hep-th/9811156.

[181] G. Ferretti and D. Martelli, "ON THE CONSTRUCTION OF GAUGE THEORIES FROM NONCRITICAL TYPE 0 STRINGS", Adv.Theor.Math.Phys. 3 (1999) 119, hep-th/9811208.

[182] A. Armoni, E. Fuchs and J. Sonnenshein, "CONFINEMENT IN 4-D YANG-MILLS THEORIES FROM NONCRITICAL TYPE 0 STRING THEORY", JHEP 9906 (1999) 027, hep-th/9903090.

[183] A. Uranga," COMMENTS ON NON-SUPERSYMMETRIC ORIENTFOLDS AT STRONG COUPLING", hep-th/9912145.

[184] S. Nojiri and S.D. Odintsov, "TWO BOUNDARIES ADS / CFT CORRESPONDENCE IN DILATONIC GRAVITY", Phys.Lett. B449 (1999) 39, hep-th/9812017.

[185] A. Kehagias and K. Sfetsos, "ON RUNNING COUPLINGS IN GAUGE THEORIES FROM TYPE IIB SUPERGRAVITY", Phys.Lett. B454 (1999) 270, hep-th/9902125.

[186] L. Girardello, M. Petrini, M. Porrati and A. Zaffaroni, "CONFInEMENT AND CONDENSATES WITHOUT FINE TUNING IN SUPERGRAVITY DUALS OF GAUGE THEORIES”, JHEP 9905 (1999) 026, hep-th/9903026.

[187] S. Nojiri and S.D. Odintsov, "RUNNING GAUGE COUPLING AND QUARK - ANTI-QUARK POTENTIAL FROM DILATONIC GRAVITY", Phys.Lett. B458 (1999) 226, hep-th/9904036.

[188] R. de Mello Koch, A.Paulin-Campbell and J. Rodriques, "NONHOLOMORPHIC CORRECTIONS FROM THREE-BRANES IN F THEORY”, Phys.Rev. D60 (1999) 106008, hep-th/9903029.

[189] K. Ghoroku, "YANG-MILLS THEORY FROM NON-CRITICAL STRING", J.Phys. G26 (2000) 233 , hep-th/9907143.

[190] J. Distler and F. Zamora, "NONSUPERSYMMETRIC CONFORMAL FIELD THEORIES FROM STABLE ANTI-DE SITTER SPACES", Adv.Theor.Math.Phys. 2 (1999) 1405, hep-th/9810206.

[191] A. Karch, D. Lüst and A. Miemic, "NEW N=1 SUPERCONFORMAL FIELD THEORIES AND THEIR SUPERGRAVITY DESCRIPTION", Phys.Lett. B454 (1999) 265, hep-th/9901041. 
[192] K. Behrndt and D. Lüst, "BRANES, WAVES AND ADS ORBIFOLDS", JHEP 9907 (1999) 019, hep-th/9905180.

[193] H. Liu and A.A. Tseytlin, "D3-BRANE D INSTANTON CONFIGURATION AND N=4 SUPER YM THEORY IN CONSTANT SELFDUAL BACKGROUND", Nucl.Phys. B553 (1999) 231, hepth/9903091.

[194] N. Constable and R.C. Myers, "EXOTIC SCALAR STATES IN THE ADS / CFT CORRESPONDENCE", JHEP 9911 (1999) 020, hep-th/9905081.

[195] S. Nojiri and S.D. Odintsov, "CURVATURE DEPENDENCE OF RUNNING GAUGE COUPLING AND CONFINEMENT IN ADS / CFT CORRESPONDENCE", Phys.Rev. D61 (2000) 044014, hep-th/9905200.

[196] L. Girardello, M. Petrini, M. Porrati and A. Zaffaroni, "THE SUPERGRAVITY DUAL OF N=1 SUPER YANG-MILLS THEORY", hep-th/9909047.

[197] A. Burinskii, "DILATONIC ADS-KERR SOLUTION TO ADS/CFT CORRESPONDENCE", Phys.Rev. D61 (2000) 107501, hep-th/9908198.

[198] L. Susskind and E. Witten, "THE HOLOGRAPHIC BOUND IN ANTI-DE SITTER SPACE", hep-th/9805114.

[199] A.W. Peet and J. Polchinski, "UV / IR RELATIONS IN ADS DYNAMICS", Phys.Rev. D59 (1999) 065011, hep-th/9809022.

[200] T.R. Taylor and G. Veneziano, "STRINGS AND D = 4", Phys.Lett. 212B (1988) 147.

[201] I. Antoniadis, "A POSSIBLE NEW DIMENSION AT A FEW TEV", Phys.Lett. B246 (1990) 377.

[202] E. Witten, "PHASE TRANSITIONS IN M THEORY AND F THEORY", Nucl.Phys. B471 (1996) 135 , hep-th/9603150.

[203] J. Lykken, "WEAK SCALE SUPERSTRINGS", Phys.Rev. D54 (1996) 3693.

[204] K.R. Dienes, E. Dudas and T. Gherghetta, "EXTRA SPACE-TIME DIMENSIONS AND UNIFICATION", Phys.Lett. B436 (1998) 55, hep-ph/9803466.

[205] C. Bachas, "UNIFICATION WITH LOW STRING SCALE", JHEP 23 (1998)9811, hep$\mathrm{ph} / 9807415$.

[206] J. Maldacena, "WILSON LOOPS IN LARGE N FIELD THEORIES", Phys.Rev.Lett. 80 (1998) 4859, hep-th/9803002.

[207] D.J. Gross and H. Ooguri, "ASPECTS OF LARGE N GAUGE THEORY DYNAMICS AS SEEN BY STRING THEORY" Phys.Rev. D58 (1998) 106002, hep-th/9805129. 
[208] A. Tseytlin, "TYPE IIB INSTANTON AS A WAVE IN TWELVE-DIMENSIONS", Phys.Rev.Lett. 78 (1997) 1864, hep-th /9612164.

[209] S. Gubser, I. Klebanov and A. Tseytlin, "COUPLING CONSTANT DEPENDENCE IN THE THERMODYNAMICS OF N=4 SUPERSYMMETRIC YANG-MILLS THEORY", Nucl.Phys. B534 (1998) 202, hep-th/9805156

[210] A.A. Tseytlin and S. Yankielowicz, "FREE ENERGY OF N=4 SUPER YANG-MILLS IN HIGGS PHASE AND NONEXTREMAL D3-BRANE INTERACTIONS", Nucl.Phys. B541 (1999) 145, hep-th/9809032.

[211] S. Gubser, I. Klebanov and A. Peet, "ENTROPY AND TEMPERATURE OF BLACK 3BRANES", Phys.Rev. D54 (1996) 3915.

[212] S.W. Hawking and D.N. Page, "THERMODYNAMICS OF BLACK HOLES IN ANTI-DE SITTER SPACE", Comm.Math.Phys. 87 (1983) 577.

[213] S. Nojiri and S.D. Odintsov, "RUNNING GAUGE COUPLING AND QUARK - ANTI-QUARK POTENTIAL IN NON-SUSY GAUGE THEORY AT FINITE TEMPERATURE FROM IIB SG / CFT CORRESPONDENCE", Phys.Rev. D61(2000) 024027, hep-th/9906216.

[214] V. Skalozub, "EFFECTIVE COUPLING CONSTANTS IN GAUGE THEORIES AT HIGH TEMPERATURE", Int.J.Mod.Phys. A11 (1996) 5643.

[215] E. Witten, "ANTI-DE SITTER SPACE, THERMAL PHASE TRANSITION, AND CONFINEMENT IN GAUGE THEORIES", Adv.Theor.Math. 2 (1998) 505, hep-th/9803131.

[216] N. Itzhaki, J.M. Maldacena, J. Sonnenschein and S. Yankielowicz, "SUPERGRAVITY AND THE LARGE N LIMIT OF THEORIES WITH SIXTEEN SUPERCHARGES", Phys.Rev. D58 (1998) 046004, hep-th/9802042.

[217] S.-J. Rey, S. Theisen and J. Yee, "WILSON-POLYAKOV LOOP AT FINITE TEMPERATURE IN LARGE N GAUGE THEORY AND ANTI-DE SITTER SUPERGRAVITY”, Nucl.Phys. B527 (1998) 171, hep-th/9803135.

[218] A. Brandhuber, N. Itzhaki, J. Sonnenschein, S. Yankielowicz, "WILSON LOOPS IN THE LARGE N LIMIT AT FINITE TEMPERATURE” Phys.Lett. B434 (1998) 36, hep-th/9803137.

[219] A. Chamblin, R. Emparan, C.V. Johnson and R.C. Myers, "LARGE N PHASES, GRAVITATIONAL INSTANTONS AND THE NUTS AND BOLTS OF ADS HOLOGRAPHY", Phys.Rev. D59 (1999) 064010, hep-th/9808177.

[220] S.W. Hawking, C.J. Hunter and D.N. Page, "NUT CHARGE, ANTI-DE SITTER SPACE AND ENTROPY", Phys.Rev. D59 (1999) 044033, hep-th/9809035.

[221] J.L.F. Barbon, I.I. Kogan and E. Rabinovici, "ON STRINGY THRESHOLDS IN SYM / ADS THERMODYNAMICS", Nucl.Phys. B544 (1999) 104, hep-th/9809033. 
[222] A. Hashimoto and Y. Oz, "ASPECTS OF QCD DYNAMICS FROM STRING THEORY", Nucl.Phys. B548 (1999) 167, hep-th/9809106.

[223] S. Kalayana Rama and B. Sathiapalan, "THE HAGEDORN TRANSITION, DECONFINEMENT AND THE ADS / CFT CORRESPONDENCE", Mod.Phys.Lett. A13 (1998) 3137, hep-th/9810069.

[224] A.W. Peet and S.F. Ross, "MICROCANONICAL PHASES OF STRING THEORY ON ADS(M) X S**N", JHEP 12 (1998) 020, hep-th/9810200.

[225] Y. Kinar, E. Schreiber and J. Sonnenschein, "Q ANTI-Q POTENTIAL FROM STRINGS IN CURVED SPACE-TIME: CLASSICAL RESULTS”, Nucl.Phys. B566 (2000) 103, hep-th/9811192.

[226] J. Greensite and P. Olesen, "WORLD SHEET FLUCTUATIONS AND THE HEAVY QUARK POTENTIAL IN THE ADS / CFT APPROACH", JHEP 04 (1999) 001, hep-th/9901057.

[227] K. Landsteiner, "STRING CORRECTIONS TO THE HAWKING-PAGE PHASE TRANSITION", Mod.Phys.Lett. A14 (1999) 379, hep-th/9901143.

[228] J. Ellis, A. Ghosh and N.E. Mavromatos, "ON THE THERMODYNAMICS OF A GAS OF ADS BLACK HOLES AND THE QUARK HADRON PHASE TRANSITION", Phys.Lett. B454 (1999) 193, hep-th/9902190.

[229] M. Cvetic an S.S. Gubser, "PHASES OF R CHARGED BLACK HOLES, SPINNING BRANES AND STRONGLY COUPLED GAUGE THEORIES", JHEP 04 (1999) 024, hep-th/9902195.

[230] E. Kiritsis and T.R. Taylor, "THERMODYNAMICS OF D-BRANE PROBES", hep-th/9906048.

[231] C. Burgess, N. Constable and R.C. Myers, "THE FREE ENERGY OF N=4 SUPER YANG-MILLS AND THE ADS / CFT CORRESPONDENCE”, JHEP 9908 (1999) 017, hep-th/9907188.

[232] B. Sundborg, "THE HAGEDORN TRANSITION, DECONFINEMENT AND N=4 SYM THEORY", hep-th/9908001.

[233] K. Landsteiner and E. Lopez, "PROBING THE STRONG COUPLING LIMIT OF LARGE N SYM ON CURVED BACKGROUNDS”, JHEP 9909 (1999) 006, hep-th/9908010.

[234] T. Harmark and N. Obers, "THERMODYNAMICS OF SPINNING BRANES AND THEIR DUAL GAUGE THEORIES", JHEP 0001 (2000) 008, hep-th/9910036.

[235] S. Nojiri and S.D. Odintsov, "STRONG COUPLING LIMIT OF N=2 SCFT FREE ENERGY AND HIGHER DERIVATIVE ADS/CFT CORRESPONDENCE", Phys.Lett. B471 (1999) 155, hep-th/9908065.

[236] M. Nishimura and Y. Tanii, "SUPER WEYL ANOMALIES IN THE ADS / CFT CORRESPONDENCE", Int.J.Mod.Phys. A14 (1999) 3731, hep-th/9904010.

[237] V. Balasubramanian and P. Kraus, "A STRESS TENSOR FOR ANTI-DE SITTER GRAVITY", Commun.Math.Phys. 208 (1999) 413, hep-th/9902121. 
[238] W. Muck and K.S. Viswanathan, "COUNTERTERMS FOR THE DIRICHLET PRESCRIPTION OF THE ADS / CFT CORRESPONDENCE", hep-th/9905046.

[239] P. Mansfield and D. Nolland, "ONE LOOP CONFORMAL ANOMALIES FROM ADS / CFT IN THE SCHRODINGER REPRESENTATION", JHEP 9907 (1999) 028, hep-th/9906054.

[240] J. Ho," HOLOGRAFIC COUNTERTERMS ACTIONS AND ANOMALIES FOR ASYMPTOTIC AdS AND FLAT SPACES", hep-th/9910124.

[241] A. Sen, "F THEORY AND ORIENTIFOLDS", Nucl.Phys. B475 (1996) 562, hep-th/9605150.

[242] T. Banks, M.R. Douglas and N. Seiberg, "PROBING F THEORY WITH BRANES", Phys.Lett. B387 (1996) 278, hep-th/9605199.

[243] O. Aharony, C. Sonnenstein, S. Yankielowicz and S. Theisen, "FIELD THEORY QUESTIONS FOR STRING THEORY ANSWERS", Nucl.Phys. B493 (1997) 177, hep-th/9611222.

[244] M.R. Douglas, D.A. Lowe and J.H. Schwarz, "PROBING F THEORY WITH MULTIPLE BRANES", Phys.Lett. B394 (1997) 297, hep-th/9612062.

[245] O. Aharony, A. Fayyazuddin and J.M. Maldacena, "THE LARGE N LIMIT OF N=2, N=1 FIELD THEORIES FROM THREE-BRANES IN F THEORY", JHEP 9807 (1998) 013, hep-th/9806159.

[246] A. Fayyazuddin and M. Spalinski, "LARGE N SUPERCONFORMAL GAUGE THEORIES AND SUPERGRAVITY ORIENTIFOLDS", Nucl.Phys. B535 (1998) 219, hep-th/9805096.

[247] A.A. Tseytlin, "HETEROTIC TYPE I SUPERSTRING DUALITY AND LOW-ENERGY EFFECTIVE ACTIONS", Nucl.Phys. B467 (1996) 383, hep-th/9512081.

[248] N. Seiberg and E. Witten, "ELECTRIC - MAGNETIC DUALITY, MONOPOLE CONDENSATION, AND CONFINEMENT IN N=2 SUPERSYMMETRIC YANG-MILLS THEORY", Nucl.Phys. B426 (1994) 19, hep-th/9407087.

[249] T. Damour and A.M. Polyakov, "THE STRING DILATON AND A LEAST COUPLING PRINCIPLE", Nucl.Phys. B423 (1994) 532.

[250] I. Antoniadis, P.O. Mazur and E. Mottola, "CONFORMAL INVARIANCE AND COSMIC BACKGROUND RADIATION", Phys.Rev.Lett. 79 (1997) 14. 